\title{
Conjuntos de continuidade sequencial fraca para polinômios em espaços de Banach
}

\author{
Pedro Levit Kaufmann
}

\author{
dissertação de mestrado \\ INSTITUTO DE MATEMÁTICA E ESTATÍSTICA DA \\ UNIVERSIDADE DE SÃO PAULO
}

\author{
Área de concentração: Análise \\ Orientadora: Profa. Dra. Mary Lilian Lourenço
}

Durante o programa de mestrado, o autor obteve

apoio financeiro do CNPq e da CAPES 


\title{
Resumo
}

Esta dissertação tem por objetivo a apresentação de um estudo em espaços de Banach sobre os conjuntos nos quais determinados polinômios homogêneos contínuos são fracamente sequencialmente contínuos. Algumas propriedades desses conjuntos são estudadas

e ilustradas com exemplos, em maior parte no espaço $l_{p}$. Obtemos um fórmula para o conjunto de continuidade sequencial fraca do produto de dois polinômios e algumas consequências. Resultados mais fortes são obtidos quando restringimos nossos espaços de Banach a espaços com FDD incondicional e/ou separáveis. Os resultados estudados aqui foram obtidos por R. Aron e V. Dimant em [2].

\begin{abstract}
This work has the purpose of presenting a study on Banach spaces about sets in which determined homogeneous continuous polynomials are weakly sequentially continuous. Some properties of these sets are studied and illustrated with examples, most in the space $l_{p}$. We obtain a formula for the weak sequential continuity set of the product of two polynomials, and some consequences. Stronger results are obtained when we restrict our Banach spaces to spaces with unconditional FDD and/or separable. The results studied here were obtained by R. Aron and V. Dimant in [2].
\end{abstract}




\section{Introdução:}

O espaço dos polinômios homogêneos contínuos definidos em espaços de Banach tem sido objeto contínuo de pesquisa nos últimos anos. Um dos pontos a se destacar é o grande interesse em estabelecer relações entre um espaço de Banach E e o espaço dos polinômios definidos sobre E. Podemos observar tal fato em diversos trabalhos científicos que estudam como os polinômios portam-se em conexão com certas propriedades geométricas dos espaços de Banach onde estão definidos e de propriedades definidas em termos de polinômios. Citamos aqui alguns trabalhos, [4], [5] e [11], que abordam o referido tema.

O interesse sobre conjunto de continuidade sequencial fraca de um polinômio $P$, denotado por $C_{P}$, tem como origem o fato de que nem todo polinômio é fracamente sequencialmente contínuo em todo domínio ou em ponto algum, como se verifica logo ao início do capítulo 3. Algumas propriedades interessantes são obtidas quando $P$ é homogêneo. Uma fórmula para $C_{P . Q}$ nos permite analisar a irredutibilidade de determinados polinômios, estabelecendo assim uma interessante conexão entre propriedades topológicas e algébricas de polinômios. Os resultados sobre o assunto aqui expostos se encontram em [2]

A seguir descrevemos os assuntos abordados em cada capítulo.

No capítulo 1, são apresentados primeiramente alguns resultados básicos de análise funcional usados no decorrer deste trabalho.

A seguir há uma introdução aos espaços de Banach com FDD incondicional, com algumas propriedades, inclusive a de que espaços deste tipo são separáveis.

Também no capítulo 1 desenvolvemos a teoria básica de topologia fraca, estabelecendose o conceito de convergência fraca, que é central neste trabalho. É apresentada uma caracterização de convergência fraca em espaços do tipo $l_{p}$, útil para o desenvolvimento de certos exemplos.

No capítulo 2 estudamos polinômios contínuos, enfatizando os polinômios homogêneos. Apresentamos ainda uma versão análoga ao Teorema de Banach-Steinhaus para polinômios homogêneos. Desenvolve-se também a técnica de complexificação de um polinômio em um espaço de Banach real. Algumas propriedades dos polinômios fracamente sequencialmente contínuos também são estudadas, e é enunciado sem demonstração o Teorema de Pitt, que terá grande importância para compreensão de certos exemplos no capítulo 3. 
Na primeira seção do capítulo 3 definimos o conjunto $C_{P}$ e estudamos suas propriedades básicas. Os resultados nesta seção foram obtidos por R. Aron e V. Dimant em [2]. Apresentamos uma fórmula para $C_{P . Q}$, e algumas aplicações desta fórmula para irredutibilidade de polinômios. Estudamos certas questões envolvendo $C_{P}$ que são colocadas e parcialmente respondidas em [2], no caso em que $E$ é um espaço de Banach qualquer.

Na segunda seção é estudado o conjunto $C_{P}$ para espaços de Banach particulares, a saber, espaços com FDD incondicional e/ou separáveis. Os resultados desta seção se encontram em [2] e em um trabalho de V. Dimant e R. Gonzalo [6]. Definimos os polinômios diagonais em espaços com FDD incondicional. Para estes espaços, são novamente discutidas as questões colocadas ao final da seção anterior. 


\section{Sumário}

1 Resultados preliminares $\quad 6$

1.1 Decomposição de Schauder incondicional . . . . . . . . . . . . . . . 10

1.2 Topologia fraca . . . . . . . . . . . . . . . . . . . . . . . . . . 19

2 Polinômios homogêneos contínuos $\quad 25$

2.1 Aplicações multilineares . . . . . . . . . . . . . . . . . . 25

2.2 Aplicações Multilineares Simétricas . . . . . . . . . . . . . . . 33

2.3 Polinômios . . . . . . . . . . . . . . . . . . . . . . . . . . 35

2.4 Polinômios fracamente sequencialmente contínuos . . . . . . . . . . . 44

2.5 Complexificação de polinômios . . . . . . . . . . . . . . . . 46

3 O conjunto $C_{P} \quad 51$

3.1 Propriedades gerais de $C_{P} \ldots \ldots \ldots \ldots \ldots$

$3.2 C_{P}$ para espaços de Banach particulares . . . . . . . . . . 75 


\title{
Notações
}

\author{
$\mathbb{N}_{0} \quad \mathbb{N} \cup\{0\}$ \\ $\mathbb{K}$ corpo de escalares, reais ou complexos \\ $X, Y \quad \mathbb{K}$-espaços vetoriais ou espaços normados \\ $E, F$ espaços de Banach \\ [M] espaço gerado pelo conjunto $M$ \\ $\mathcal{L}(E ; F)$ transformações lineares contínuas de $E$ em $F$ \\ $X^{*}$ funcionais lineares de $X$ \\ $E^{\prime}$ espaço dual de $\mathrm{E}$ \\ $e^{k}$ sequência de escalares que vale 1 na $k$-ésima coordenada e zero nas demais \\ $x_{n} \stackrel{w}{\rightarrow} x \quad x_{n}$ converge fracamente a $x$ \\ $\mathcal{L}_{a}\left({ }^{m} X ; Y\right)$ transformações m-lineares de $X$ em $Y$ \\ $\mathcal{L}\left({ }^{m} E ; F\right)$ transformações m-lineares contínuas de $E$ em $F$ \\ $\mathcal{L}\left({ }^{m} E\right) \quad \mathcal{L}\left({ }^{m} E ; \mathbb{K}\right)$ \\ $\mathcal{L}_{a s}\left({ }^{m} X ; Y\right)$ transformações m-lineares simétricas de $X$ em $Y$ \\ $\mathcal{L}_{s}\left({ }^{m} E ; F\right)$ transformações m-lineares simétricas contínuas de $E$ em $F$ \\ $\mathcal{L}_{s}\left({ }^{m} E\right) \quad \mathcal{L}_{s}\left({ }^{m} E ; \mathbb{K}\right)$ \\ $\mathcal{P}_{a}\left({ }^{m} X ; Y\right)$ polinômios m-homogêneos de $X$ em $Y$ \\ $\mathcal{P}\left({ }^{m} E ; F\right)$ polinômios m-homogêneos contínuos de $E$ em $F$ \\ $\mathcal{P}\left({ }^{m} E\right) \quad \mathcal{P}\left({ }^{m} E ; \mathbb{K}\right)$ \\ $\mathcal{P}_{a}(X ; Y)$ polinômios de $X$ em $Y$ \\ $\mathcal{P}(E ; F)$ polinômios contínuos de $E$ em $F$ \\ $\mathcal{P}(E) \quad \mathcal{P}(E ; \mathbb{K})$ \\ $\mathcal{P}_{w s c}\left({ }^{m} E\right)$ polinômios m-homogêneos fracamente sequencialmente contínuos \\ $\mathcal{P}_{\text {wsc } 0}\left({ }^{m} E\right)$ polinômios m-homogêneos fracamente sequencialmente contínuos na origem \\ $\mathcal{D}_{J}\left({ }^{m} E\right)$ polinômios m-homogêneos contínuos diagonais com relação a $J$ \\ $C_{P}$ conjunto de continuidade sequêncial fraca do polinômio $P$
}




\section{Capítulo 1}

\section{Resultados preliminares}

Apresentamos inicialmente alguns resultados básicos que serão necessários no decorrer deste trabalho. Muitas afirmações ao longo deste capítulo serão feitas sem demonstração; são consideradas pré-requisitos para o estudo deste trabalho. Essas afirmações podem ser encontradas com demonstração em boa parte dos livros de análise matemática, por exemplo em [13] e [15]. Serão utilizados também notações e resultados básicos de topologia. Lembremos que, quando $X$ é espaço normado e $F$ é espaço de Banach, $\mathcal{L}(X ; F)$ munido da norma $\|T\| \doteq \sup \{\|T(x)\|:\|x\| \leq 1\}$ é um espaço de Banach.

Ao longo do estudo, os seguintes três resultados fundamentais $(1.1,1.5$ e 1.6) serão frequentemente referidos:

Teorema 1.1 (Teorema de Hahn-Banach). Sejam X um espaço normado, S um subespaço qualquer de $X$ e $\gamma \in S^{\prime}$. Então existe $\bar{\gamma} \in X^{\prime}$ tal que para cada $x \in S$ temos que $\bar{\gamma}(x)=\gamma(x) e\|\bar{\gamma}\|=\|\gamma\|$.

Corolário 1.2. Sejam $X$ um espaço normado, $S$ um subespaço fechado de $X$ e $x \in X \backslash S$.

Então existe $\gamma \in X^{\prime}$ satisfazendo $\gamma(S)=\{0\}, \gamma(x)=d(x, S)=\inf \{\|x-y\|: y \in S\}$ $e\|\gamma\|=1$.

\section{Demonstração:}

Vamos definir um funcional $f$ em $S+[x]$ por $f(y+\lambda x) \doteq \lambda d(x, S)$, para cada $y \in$ $S, \lambda \in \mathbb{K}$. Então $f(x)=d(x, S)$ e para cada $y \in S, f(y)=0$.

Para cada $y \in S$ e cada $\lambda \in \mathbb{K}$ não nulo temos que

$$
|f(y+\lambda x)|=|\lambda| d(x, S) \leq|\lambda|\left\|x-\left(-\lambda^{-1} y\right)\right\|=\|y+\lambda x\|,
$$


de forma que $f$ é contínuo e $\|f\| \leq 1$. Para cada $y \in S$,

$$
\|f\|\|x-y\| \geq|f(x-y)|=d(x, S)
$$

e desta forma

$$
\|f\| d(x, S)=\|f\| \inf \{\|x-y\|: y \in S\} \geq d(x, S) .
$$

Sendo $d(x, S)>0$, temos que $\|f\|=1$. Portanto pelo Teorema de Hahn-Banach (1.1) existe $\gamma \in X^{\prime}$ satisfazendo $\gamma(S)=\{0\}, \gamma(x)=d(x, S)=\inf \{\|x-y\|: y \in S\}$ e $\|\gamma\|=1$, como queríamos.

Corolário 1.3. Sejam $X$ um espaço normado e $x \in X$ não nulo. Então existe $\gamma \in X^{\prime}$ tal que $\gamma(x)=\|x\| e\|\gamma\|=1$.

\section{Demonstração:}

Considerando $S=\{0\}$, podemos usar o corolário 1.2 para afirmar que existe $\gamma \in X^{\prime}$ satisfazendo $\gamma(x)=\|x\|$ e $\|\gamma\|=1$.

Corolário 1.4. Seja $X$ um espaço normado, e sejam $x, y \in X$ distintos. Então existe $\gamma \in X^{\prime}$ tal que $\gamma(x) \neq \gamma(y)$.

\section{Demonstração:}

$x-y \neq 0$. Então considerando de novo $S=\{0\}$, pelo corolário 1.2 existe $\gamma \in X^{\prime}$ satisfazendo $\gamma(x)-\gamma(y)=\gamma(x-y)=\|x-y\|>0$. Assim, $\gamma(x) \neq \gamma(y)$.

Teorema 1.5 (Teorema da aplicação aberta). Sejam E e F espaços de Banach e $T \in$ $\mathcal{L}(E ; F)$ sobrejetora. Então para cada subconjunto aberto $U$ de E temos que $T(U)$ é um aberto de $F$.

Teorema 1.6 (Princípio da limitação uniforme). Sejam E um espaço de Banach, Y um espaço normado e $\mathcal{F}$ uma família não vazia de elementos de $\mathcal{L}(E ; Y)$. Se $\sup \{\|T(x)\|$ : $T \in \mathcal{F}\}$ é finito para cada $x \in E$, então $\sup \{\|T\|: T \in \mathcal{F}\}$ é finito.

Como consequência do princípio da limitação uniforme temos o seguinte: 
Teorema 1.7 (Teorema de Banach-Steinhaus). Sejam E um espaço de Banach, Y um espaço normado e $\left(T_{n}\right)_{n}$ uma sequência em $\mathcal{L}(E ; Y)$.

Então, se para cada $x \in E$ existir $\lim _{n \rightarrow \infty} T_{n}(x)$, teremos que $T(x) \doteq \lim _{n \rightarrow \infty} T_{n}(x)$ é uma transformação linear contínua de $E$ em $Y$.

\section{Demonstração:}

$T$ é linear, pois para cada $x, y \in E$ e cada $\lambda \in \mathbb{K}$ temos que

$T(x+\lambda y)=\lim _{n \rightarrow \infty} T_{n}(x+\lambda y)=\lim _{n \rightarrow \infty}\left(T_{n}(x)+\lambda T_{n}(y)\right)=\lim _{n \rightarrow \infty} T_{n}(x)+\lambda \lim _{n \rightarrow \infty} T_{n}(y)=T(x)+\lambda T(y)$.

Como $\sup \left\{\left\|T_{n}(x)\right\|: n \in \mathbb{N}\right\}$ é finito para cada $x \in E$, então pelo princípio da limitação uniforme existe $M>0$ tal que $\sup \left\{\left\|T_{n}\right\|: n \in \mathbb{N}\right\}<M$. Então para cada $x \in E$ com $\|x\| \leq 1$ temos que $\left\|T_{n}(x)\right\|<M$, e assim $\|T(x)\| \leq M$. Portanto $T$ é contínua.

Versões mais gerais desses Teoremas podem ser encontradas nas referências citadas ao início do capítulo.

Vamos precisar também dos dois próximos resultados sobre funcionais lineares. Recordemos que um hiperplano em $X$ por definição é um subespaço próprio de $X$ maximal, isto é, um subespaço próprio $H$ de $X$ é um hiperplano se para cada subespaço $H^{\prime}$ distinto de $H$ satisfazendo $H \subset H^{\prime} \subset X$ tivermos que $H^{\prime}=X$.

Proposição 1.8. Sejam $X$ um $\mathbb{K}$-espaço vetorial e $H$ um hiperplano em $X$. Então existe $\gamma \in X^{*}$ tal que $\gamma^{-1}(0)=H$.

Reciprocamente, se $\gamma$ é um funcional linear não nulo de $X$, então $H \doteq \gamma^{-1}(0)$ é um hiperplano.

\section{Demonstração:}

Sejam $H$ hiperplano de $X$ e $v \in X \backslash H$. Temos que $X=H \oplus[v]$, isto é, cada $x \in X$ se escreve de maneira única na forma $x=h_{x}+\lambda_{x} v$, onde $h_{x} \in H$ e $\lambda_{x} \in \mathbb{K}$.

Definimos $\gamma: X \rightarrow \mathbb{K}$ por $\gamma(x) \doteq \lambda_{x}$. É facil ver que $\gamma$ é linear, e vale que $\gamma^{-1}(0)=H$ pois $\gamma(x)=0 \Leftrightarrow \lambda_{x}=0 \Leftrightarrow x=h_{x}+0 v \Leftrightarrow x \in H$.

Seja agora $\gamma$ um funcional linear não nulo de $X$, e seja $v \in X$ tal que $\gamma(v)=1$.

Cada $y \in X$ se escreve como $y=(\underbrace{y-\gamma(y) v}_{\in \gamma^{-1}(0)})+\gamma(y) v$. Agora $\gamma^{-1}(0)$ é um subespaço próprio de $X$, mas cada $y \in X$ se escreve como $y=w+k v$, com $w \in \gamma^{-1}(0)$, de forma 
que $X=\gamma^{-1}(0)+[v]$. Logo, $\gamma^{-1}(0)$ é hiperplano.

Proposição 1.9. Sejam $X$ um $\mathbb{K}$-espaço vetorial e $\gamma, \gamma_{1}, \ldots, \gamma_{n} \in X^{*}$. Então $\cap_{j=1}^{n} \gamma_{j}^{-1}(0) \subset$ $\gamma^{-1}(0)$ se e somente se existem $\alpha_{1}, \ldots, \alpha_{n} \in \mathbb{K}$ tais que $\gamma=\sum_{j=1}^{n} \alpha_{j} \gamma_{j}$.

\section{Demonstração:}

Uma das implicações é imediata. Para mostrar a outra, definamos $T \in \mathcal{L}_{a}\left(X ; \mathbb{K}^{n}\right)$ por

$$
T(x) \doteq\left(\gamma_{1}(x), \ldots, \gamma_{n}(x)\right), \forall x \in X
$$

Observe que para cada $x \in T^{-1}(0)$ temos que $x \in \cap_{j=1}^{n} \gamma_{j}^{-1}(0)$, e desta forma por hipótese $x \in \gamma^{-1}(0)$.

Desta forma, podemos definir $\psi: T(X) \subset \mathbb{K}^{n} \rightarrow \mathbb{K}$ como sendo, para cada $T(x) \in$ $T(X)$

$$
\psi(T(x)) \doteq \gamma(x)
$$

$\psi$ está bem definida, pois para cada $x, y \in X$, temos que

$$
T(x)=T(y) \Leftrightarrow T(x-y)=0 \Leftrightarrow x-y \in T^{-1}(0) \Leftrightarrow x-y \in \gamma^{-1}(0) \Leftrightarrow \gamma(x)=\gamma(y) .
$$

Sejam $T(x)$ e $T(y) \in T(X)$ e $\lambda \in \mathbb{K}$. Então

$$
\psi(T(x)+\lambda T(y))=\psi(T(x+\lambda y))=\gamma(x+\lambda y)=\gamma(x)+\lambda \gamma(y)=\psi(T(x))+\lambda \psi(T(y))
$$

logo $\psi$ é linear, e é contínua por estar definida em um espaço de dimensão finita.

Aplicando o Teorema de Hahn-Banach (1.1), sabemos que existe uma aplicação linear contínua $\widetilde{\psi}: \mathbb{K}^{n} \rightarrow \mathbb{K}$ tal que, para todo $x \in X, \widetilde{\psi}(T(x))=\psi(T(x))$.

Seja $\left\{e_{1}, \ldots, e_{n}\right\}$ a base canônica de $\mathbb{K}^{n}$. Então para cada $x \in X$

$$
\gamma(x)=\psi(T(x))=\widetilde{\psi}(T(x))=\widetilde{\psi}\left(\gamma_{1}(x), \ldots, \gamma_{n}(x)\right)=\widetilde{\psi}\left(\sum_{j=1}^{n} \gamma_{j}(x) e_{j}\right)=\sum_{j=1}^{n} \widetilde{\psi}\left(e_{j}\right) \gamma_{j}(x)
$$

e portanto $\gamma=\sum_{j=1}^{n} \widetilde{\psi}\left(e_{j}\right) \gamma_{j}$, como queríamos. 


\subsection{Decomposição de Schauder incondicional}

A definição de decomposição de Schauder incondicional em um espaço de Banach é semelhante à definição de base de Schauder incondicional, como veremos a seguir. Primeiro, vamos definir base de Schauder. Para tal, precisamos estabelecer o conceito de convergência incondicional de séries:

Definição 1.10. Dizemos que uma série $\sum_{n=1}^{\infty} x_{n}$ em E é incondicionalmente convergente $\boldsymbol{a} x$ se para cada $\sigma$ permutação dos naturais tivermos que $\sum_{n=1}^{\infty} x_{\sigma(n)}=x$.

Exemplo 1.11. A série em $\mathbb{R}$ dada por $\sum_{n=1}^{\infty} \frac{(-1)^{n}}{n}$ é convergente mas não é incondicionalmente convergente.

Definição 1.12. Uma sequência $\left(x_{n}\right)_{n}$ em um espaço de Banach E é dita uma base de Schauder para E se para cada $x \in E$ existe uma única sequência de escalares $\left(\lambda_{n}\right)_{n}$ tal que

$$
x=\sum_{n=1}^{\infty} \lambda_{n} x_{n}
$$

Se além disso a série converge incondicionalmente para cada $x \in E$, dizemos que $\left(x_{n}\right)_{n}$ é uma base de Schauder incondicional para E.

Observação: Decorre diretamente da definição acima que, se $\left(x_{n}\right)_{n}$ é uma base de Schauder para um espaço de Banach $E$, então $\overline{\left[\left\{x_{n}\right\}_{n \in \mathbb{N}}\right]}=E$.

Exemplo 1.13. A base clássica de Schauder para $C[0,1]$ é uma base de Schauder que não é incondicional.

A base clásica de Schauder é dada pela sequência $\left(s_{n}\right)_{n=0}^{\infty}$ de elementos de $C[0,1]$, dada por $s_{0}(t) \doteq 1, s_{1}(t) \doteq t$ e para $n \geq 2$

$$
s_{n}(t)= \begin{cases}2^{m}\left(t-\left(\frac{2 n-2}{2^{m}}-1\right)\right) & \text { se } \frac{2 n-2}{2^{m}}-1 \leq t<\frac{2 n-1}{2^{m}}-1 \\ 1-2^{m}\left(t-\left(\frac{2 n-1}{2^{m}}-1\right)\right) & \text { se } \frac{2 n-1}{2^{m}}-1 \leq t<\frac{2 n}{2^{m}}-1 \\ 0 & \text { caso contrário }\end{cases}
$$

onde $m$ é o natural que satisfaz $2^{m-1}<n \leq 2^{m}$. A figura abaixo ilustra essa sequência: 

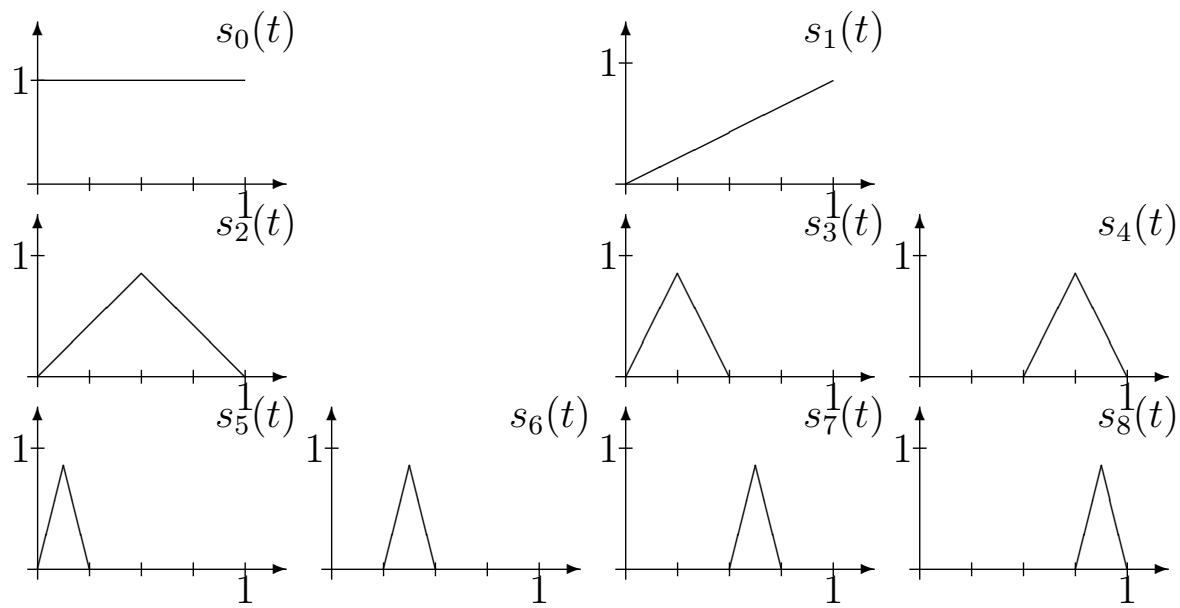

Esse exemplo é de [21], artigo no qual foi introduzida a noção de base de Schauder.

Exemplo 1.14. Se $E=c_{o}$ ou $l_{p}$ com $1 \leq p<\infty$, a sequência $\left(e^{n}\right)_{n}$ é base de Schauder para E. Mas $\left(e^{n}\right)_{n}$ não é base de Schauder para $l_{\infty}$.

Verifiquemos que $\left(e^{n}\right)_{n}$ é base de Schauder incondicional para $l_{p}$. Para cada $x=$ $\left(x_{1}, x_{2}, \ldots\right) \in l_{p}$ temos que $\sum_{n=1}^{\infty}\left|x_{n}\right|^{p}$ converge, de forma que

$$
\left\|x-\sum_{i=1}^{N} x_{i} e^{i}\right\|_{p}=\left\|\left(0, \ldots, 0, x_{N+1}, x_{N+2}, \ldots\right)\right\|_{p}=\left(\sum_{i=N+1}^{\infty}\left|x_{i}\right|^{p}\right)^{\frac{1}{p}} \stackrel{N}{\rightarrow} 0
$$

e portanto a série $\sum_{n=1}^{\infty} x_{n} e^{n}$ converge a $x$. Assim, $\left(e^{n}\right)_{n}$ é base de Schauder para $l_{p}$. Para mostrar que essa base de Schauder é incondicional, consideremos $\sigma$ uma permutação dos naturais. Vamos definir $f: \mathbb{N} \rightarrow \mathbb{N}$ por

$$
f(n) \doteq \sup \{\sigma(j): j \in \mathbb{N}, j \leq n\} .
$$

Observe que $f(n) \stackrel{n}{\rightarrow} \infty$. Considerando a sequência de escalares $\left(x_{\sigma(n)}\right)_{n}$ temos que

$$
\left\|x-\sum_{i=1}^{N} x_{\sigma(i)} e^{\sigma(i)}\right\|_{p}=\left(\sum_{\substack{i \neq \sigma(j) \\ j=1, \ldots, N}}\left|x_{i}\right|^{p}\right)^{\frac{1}{p}} \leq\left(\sum_{i=f(N)+1}^{\infty}\left|x_{i}\right|^{p}\right)^{\frac{1}{p}} \stackrel{N}{\rightarrow} 0 .
$$

Desta forma, a série $\sum_{i=1}^{\infty} x_{i} e^{i}$ converge incondicionalmente para $x$, e podemos concluir que $\left(e^{n}\right)_{n}$ é base de Schauder incondicional para $l_{p}$. 
Verifiquemos agora que $\left(e^{n}\right)_{n}$ é base de Schauder incondicional para $c_{o}$. Seja $x=$ $\left(x_{1}, x_{2}, \ldots\right) \in c_{o}$. Dado $\epsilon>0$, existe $n_{o} \in \mathbb{N}$ tal que $\left|x_{n}\right|<\epsilon$, para cada $n \geq n_{o}$ natural. Então

$$
\left\|x-\sum_{i=1}^{n} x_{i} e^{i}\right\|_{\infty}=\left\|\left(0, \ldots, 0, x_{n+1}, x_{n+2}, \ldots\right)\right\|_{\infty} \leq \epsilon \text {, para cada } n \geq n_{o} \text { natural, }
$$

de forma que a série $\sum_{n=1}^{\infty} x_{n} e^{n}$ converge a $x$. Vamos verificar que a convergência é incondicional: seja $\sigma$ uma permutação dos naturais. Vamos definir $g: \mathbb{N} \rightarrow \mathbb{N}$ por

$$
g(n) \doteq \inf \{j \in \mathbb{N}:\{1, \ldots, n\} \subset\{\sigma(1), \ldots, \sigma(j)\}\} .
$$

Considerando novamente a sequência de escalares $\left(x_{\sigma(n)}\right)_{n}$, temos agora para cada $n \geq$ $g\left(n_{o}\right)$ que

$$
\begin{aligned}
\left\|x-\sum_{i=1}^{n} x_{\sigma(i)} e^{\sigma(i)}\right\|_{\infty} & =\sup \left\{\left\|x_{j}\right\|: j \neq \sigma(i), i=1, \ldots, n\right\} \\
& \leq \sup \left\{\left\|x_{j}\right\|: j>n_{o}\right\} \leq \epsilon
\end{aligned}
$$

e assim a série converge incondicionalmente.

Para verificar que $\left(e^{n}\right)_{n}$ não é base de Schauder para $l_{\infty}$, considero $x \doteq(1,1, \ldots) \in l_{\infty}$. Para cada $\left(\lambda_{n}\right)_{n}$ sequência de escalares temos, para cada $N$ natural, que

$$
\left\|x-\sum_{i=1}^{N} \lambda_{i} e^{i}\right\|_{\infty}=\left\|\left(1-\lambda_{1}, \ldots, 1-\lambda_{N}, 1,1, \ldots\right)\right\|_{\infty} \geq 1
$$

de forma que $\sum_{n=1}^{\infty} \lambda_{n} e^{n}$ não converge $x$. Como $\left(\lambda_{n}\right)_{n}$ é arbitrária, concluimos nossa verificação.

Para definirmos decomposição de Schauder incondicional, se fará necessária a noção de projeção, dada pela definição a seguir.

Definição 1.15. Sejam um $\mathbb{K}$-espaço vetorial $X$ e $S$ um subespaço de $X$. Dizemos que $\Pi: X \rightarrow S$ é uma projeção se $\Pi$ for linear e $\Pi(\Pi(x))=\Pi(x)$, para cada $x \in X$.

A definição de base de Schauder incondicional pode ser reescrita da seguinte forma: "Seja $\left(x_{n}\right)_{n}$ uma sequência em $E$ e $E_{n} \doteq\left[x_{n}\right]$. Dizemos que $\left(x_{n}\right)_{n}$ é base de Schauder 
incondicional para $E$ se para cada $x \in E$ existir uma sequência $\left(y_{n}\right)_{n}$ em $E$ satisfazendo $y_{n} \in E_{n}, \forall n \in \mathbb{N}$, tal que $x=\sum_{n=1}^{\infty} y_{n}$ e a série converge incondicionalmente."

É natural generalizar essa definição da seguinte maneira:

Definição 1.16. Dizemos que um espaço de Banach E tem decomposição de Schauder incondicional (ou FDD incondicional), se existirem $\left\{E_{n}\right\}_{n \in \mathbb{N}}$ subespaços de $E$ de dimensão finita tais que cada $x \in E$ se escreve de maneira única como

$$
x=\sum_{i=1}^{\infty} x_{i},
$$

onde $x_{i} \in E_{i}, \forall i \in \mathbb{N}$, a série é incondicionalmente convergente e as n-ésimas projeções associadas à decomposição definidas por $\Pi_{n}(x) \doteq \sum_{i=1}^{n} x_{i}$ são contínuas.

Exemplo 1.17. Seja E com base de Schauder incondicional. Então E tem FDD incondicional.

Neste caso uma possível decomposição é a formada pelos subespaços gerados por cada elemento da base.

Em particular, os espaços $l_{p}$ com $1 \leq p<\infty$ tem FDD incondicional.

Nem sempre um espaço com FDD incondicional tem base de Schauder, como se vê pelo seguinte exemplo:

Exemplo 1.18. Seja E o espaço de Banach formado pelos operadores compactos do $l_{2}$ que têm representação triangular com respeito à base canônica, isto é, que podem ser escritos matricialmente como

$$
[T]=\left(\begin{array}{cccc}
a_{11} & a_{12} & a_{13} & \cdots \\
& a_{22} & a_{23} & \\
& & a_{33} & \\
0 & & & \ddots
\end{array}\right)
$$

Considero para cada $n \in \mathbb{N}$ o subespaço $B_{n}$ de $E$ formado pelos operadores que se escrevem como

$$
[T]=\left(\begin{array}{cccccc}
0 & \cdots & 0 & a_{1 n} & 0 & \cdots \\
\vdots & & \vdots & \vdots & \vdots & \\
0 & \cdots & 0 & a_{n n} & 0 & \cdots \\
\vdots & & \vdots & 0 & \vdots & \\
& & & \vdots & &
\end{array}\right)
$$


Não é difícil ver que $\left\{B_{n}\right\}_{n \in \mathbb{N}}$ é uma decomposição incondicional para E, e como cada $B_{n}$ tem dimensão finita $\left\{B_{n}\right\}_{n \in \mathbb{N}}$ é uma FDD incondicional para $E$. No entanto, mostra-se em [10] que este espaço não admite base de Schauder.

Observe que $\operatorname{dim} B_{n} \stackrel{n}{\rightarrow} \infty$.

Em um espaço de Banach $(E,\|\cdot\|)$ com FDD incondicional $\left\{E_{n}\right\}_{n}$ podemos introduzir uma outra norma $\|\cdot\|$, em função de $\|\cdot\|$ e de $\left\{E_{n}\right\}_{n}$, da seguinte maneira:

$$
\|\cdot\| \cdot \|: x \in E \mapsto \sup _{m \in \mathbb{N}}\left\{\left\|\sum_{n=1}^{m} x_{n}\right\|\right\} \in \mathbb{R}
$$

onde $x=\sum_{n=1}^{\infty} x_{n}, x_{i} \in E_{i}, \forall i \in \mathbb{N}$.

É evidente que para cada $x \in E,\||| x \mid\| \geq 0$ e que \|\|$x\|\|=0$ se e somente se $x=0$. Para cada $\lambda \in \mathbb{K}$

$$
\begin{aligned}
\|\lambda x\| \| & =\sup _{m \in \mathbb{N}}\left\{\left\|\lambda \sum_{n=1}^{m} x_{m}\right\|\right\} \\
& =\sup _{m \in \mathbb{N}}\left\{|\lambda|\left\|\sum_{n=1}^{m} x_{m}\right\|\right\} \\
& =|\lambda| \sup _{m \in \mathbb{N}}\left\{\left\|\sum_{n=1}^{m} x_{m}\right\|\right\}=|\lambda|\||\|x\|| .
\end{aligned}
$$

Se $x=\sum_{n=1}^{\infty} x_{n}$ e $y=\sum_{n=1}^{\infty} y_{n} \in E$,

$$
\begin{aligned}
\|\| x+y\|\| & =\sup _{m \in \mathbb{N}}\left\{\left\|\sum_{n=1}^{m} x_{m}+y_{m}\right\|\right\} \\
& \leq \sup _{m \in \mathbb{N}}\left\{\left\|\sum_{n=1}^{m} x_{m}\right\|+\left\|\sum_{n=1}^{m} x_{m}\right\|\right\} \\
& \leq \sup _{m \in \mathbb{N}}\left\{\left\|\sum_{n=1}^{m} x_{m}\right\|\right\}+\sup _{m \in \mathbb{N}}\left\{\left\|\sum_{n=1}^{m} x_{m}\right\|\right\}=\|\| x\|+\| y \|
\end{aligned}
$$

Provamos portanto que $\||\cdot|||$ é de fato uma norma sobre $E$.

Proposição 1.19. Seja $(E,\|\cdot\|)$ um espaço de Banach com FDD incondicional $\left\{E_{n}\right\}_{n}$. Então $(E,\||\cdot|\|)$ é um espaço de Banach e existe uma constante $K>0$ tal que para cada $x \in E$,

$$
\|x\| \leq\|\| x\|\mid \leq K\| x \| .
$$


Para demonstrar a proposição acima utilizaremos a seguinte consequência do Teorema da aplicação aberta (1.5):

Lema 1.20. Sejam E um $\mathbb{K}$-espaço vetorial e $\|\cdot\|_{1},\|\cdot\|_{2}$ normas sobre $E$. Se $\left(E,\|\cdot\|_{1}\right)$ $e\left(E,\|\cdot\|_{2}\right)$ forem espaços de Banach e a aplicação identidade

$$
i d:\left(E,\|\cdot\|_{1}\right) \rightarrow\left(E,\|\cdot\|_{2}\right)
$$

for contínua, então as normas $\|\cdot\|_{1} e\|\cdot\|_{2}$ são equivalentes, isto é, existem $K_{1}, K_{2}>0$ tais que

$$
K_{1}\|x\|_{1} \leq\|x\|_{2} \leq K_{2}\|x\|_{1}, \forall x \in E
$$

\section{Demonstração (do lema):}

Como por hipótese $i d:\left(E,\|\cdot\|_{1}\right) \rightarrow\left(E,\|\cdot\|_{2}\right)$ é contínua, defino $K_{2} \doteq\|i d\|>0 \mathrm{e}$ temos que

$$
\|x\|_{2} \leq K_{2}\|x\|_{1}, \forall x \in E .
$$

Pelo Teorema da aplicação aberta $(1.5)$ temos que $i d^{-1}:\left(E,\|\cdot\|_{2}\right) \rightarrow\left(E,\|\cdot\|_{1}\right)$ também é contínua.

Assim, para cada $x \in E$ posso escrever $\|x\|_{1} \leq\left\|i d^{-1}\right\|\|x\|_{2}$, e definindo $K_{1} \doteq\left\|i d^{-1}\right\|^{-1}$ temos que

$$
\|x\|_{2} \geq K_{1}\|x\|_{1}, \forall x \in E
$$

como queríamos.

\section{Demonstração (da proposição):}

Observação: No decorrer desta demonstração, as séries convergentes a que nos referirnos são convergentes na norma original de $E,\|\cdot\|$.

Seja $x \in E$. Temos que $\|x\| \leq\|\| x\|\|$, pois

$$
\|\| x\left\|=\sup _{m \in \mathbb{N}}\left\{\left\|\sum_{n=1}^{m} x_{n}\right\|\right\} \geq\right\| \sum_{n=1}^{\infty} x_{m}\|=\| x \| .
$$

Para provar que $(E,|||\cdot|||)$ é um espaço de Banach, basta provar que cada sequência de Cauchy em $(E,|| \cdot|\||)$ converge.

Seja então $\left(x^{j}\right)_{j}=\left(\sum_{n=1}^{\infty} x_{n}^{j}\right)_{j}$ uma sequência de Cauchy com relação a \|\|$\cdot\|\|$, e vamos mostrar que para cada $n \in \mathbb{N}$, a sequência $\left(x_{n}^{j}\right)_{j}$ é de Cauchy com relação a $\|\cdot\|$. Para 
cada, $j_{1}, j_{2}, k \in \mathbb{N}, k \geq 2$,

$$
\begin{aligned}
\left\|x_{k}^{j_{1}}-x_{k}^{j_{2}}\right\| & =\left\|\sum_{n=1}^{k}\left(x_{n}^{j_{1}}-x_{n}^{j_{2}}\right)-\sum_{n=1}^{k-1}\left(x_{n}^{j_{1}}-x_{n}^{j_{2}}\right)\right\| \\
& \leq 2\|\| \sum_{n=1}^{\infty}\left(x_{n}^{j_{1}}-x_{n}^{j_{2}}\right)\|\| \\
& =2\|\| \sum_{n=1}^{\infty} x_{n}^{j_{1}}-\sum_{n=1}^{\infty} x_{n}^{j_{2}}\|\|
\end{aligned}
$$

Assim, como $\left(x^{j}\right)_{j}$ é de Cauchy com relação a $\|||||$, temos que $\left(x_{k}^{j}\right)_{j}$ é de Cauchy com relação a $\|\cdot\|$. para cada natural $k \geq 2$, e $\left(x_{1}^{j}\right)$ também é de Cauchy com relação a $\|\cdot\|$ pois

$$
\left\|x_{1}^{j_{1}}-x_{1}^{j_{2}}\right\| \leq\left\|\mid \sum_{n=1}^{\infty}\left(x_{n}^{j_{1}}-x_{n}^{j_{2}}\right)\right\|\|=\| \sum_{n=1}^{\infty} x_{n}^{j_{1}}-\sum_{n=1}^{\infty} x_{n}^{j_{2}}\|\| .
$$

Sendo $(E,\|\cdot\|)$ completo, temos para cada $k \in \mathbb{N}$ que $\left(x_{k}^{j}\right)_{j}$ converge para $x_{k}$ em $E$. Vamos mostrar que $\sum_{n=1}^{\infty} x_{n}$ converge e que a sequência $\left(x^{j}\right)_{j}$ converge a $\sum_{n=1}^{\infty} x_{n}$ na norma ||$|\cdot|||$.

Seja $\epsilon>0$. Como $\left(x^{j}\right)_{j}$ é de Cauchy com relação a \|\|$\cdot\|\|$, existe $j_{\epsilon} \in \mathbb{N}$ tal que, se $j_{1}, j_{2} \in \mathbb{N}, j_{1}, j_{2} \geq j_{\epsilon}$, então para cada $m \in \mathbb{N}$ vale

$$
\left\|\sum_{n=1}^{m} x_{n}^{j_{2}}-\sum_{n=1}^{m} x_{n}^{j_{1}}\right\| \leq\left\|\sum_{n=1}^{m} x_{n}^{j_{2}}-\sum_{n=1}^{m} x_{n}^{j_{1}}\right\| \mid<\frac{\epsilon}{3} .
$$

Então, fazendo $j_{2} \rightarrow \infty$ e $j_{1}=j_{\epsilon}$, temos para cada $m \in \mathbb{N}$ que

$$
\left\|\sum_{n=1}^{m} x_{n}-\sum_{n=1}^{m} x_{n}^{j_{\epsilon}}\right\|<\frac{\epsilon}{3}
$$

e assim, para $m_{1}, m_{2} \in \mathbb{N}, m_{2} \geq m_{1}$, temos

$$
\left\|\sum_{n=m_{1}}^{m_{2}} x_{n}-\sum_{n=m_{1}}^{m_{2}} x_{n}^{j_{\epsilon}}\right\| \leq\left\|\sum_{n=1}^{m_{2}} x_{n}-\sum_{n=1}^{m_{2}} x_{n}^{j_{\epsilon}}\right\|+\left\|\sum_{n=1}^{m_{1}-1} x_{n}-\sum_{n=1}^{m_{1}-1} x_{n}^{j_{\epsilon}}\right\| \leq \frac{2 \epsilon}{3} .
$$

Seja agora $m_{\epsilon} \in \mathbb{N}$ tal que para cada $m_{1}, m_{2} \in \mathbb{N}$ com $m_{2} \geq m_{1} \geq m_{\epsilon}$ vale a desigualdade $\left\|\sum_{n=m_{1}}^{m_{2}} x_{n}^{j_{\epsilon}}\right\|<\frac{\epsilon}{3}$. Então

$$
\left\|\sum_{n=m_{1}}^{m_{2}} x_{n}\right\| \leq\left\|\sum_{n=m_{1}}^{m_{2}} x_{n}-\sum_{n=m_{1}}^{m_{2}} x_{n}^{j_{\epsilon}}\right\|+\left\|\sum_{n=m_{1}}^{m_{2}} x_{n}^{j_{\epsilon}}\right\|<\epsilon
$$


sempre que $m_{1}$ e $m_{2}$ são naturais com $m_{2} \geq m_{1} \geq m_{\epsilon}$, e portanto $\sum_{n=1}^{\infty} x_{n}$ converge.

Observe que a inequação 1.1 também é verdadeira para cada $m \in \mathbb{N}$ se substituirmos $j_{\epsilon}$ por um natural $j \geq j_{\epsilon}$. Isto é,

$$
\left\|\sum_{n=1}^{m}\left(x_{n}-x_{n}^{j}\right)\right\|<\frac{\epsilon}{3}, \forall m, j \in \mathbb{N}, j \geq j_{\epsilon} .
$$

Logo, tomando-se o supremo deste valor em $m$ temos que

$$
\|\| \sum_{n=1}^{\infty}\left(x_{n}-x_{n}^{j}\right)\|=\| \sum_{n=1}^{\infty} x_{n}-\sum_{n=1}^{\infty} x_{n}^{j} \| \leq \frac{\epsilon}{3}, \forall j \in \mathbb{N}, j \geq j_{\epsilon} .
$$

Então $\sum_{n=1}^{\infty} x_{n}$ é o limite da sequência $\left(x^{j}\right)_{j}$ com relação a $\|\mid \cdot\| \|$, e podemos concluir que $(E,|||\cdot|||)$ é um espaço de Banach.

Para mostrar que existe $K>0$ tal que para cada $x \in E$ temos $\|x\|\|\leq K\| x \|$, basta verificar que as normas $\|\cdot\| \mathrm{e}\|\| \cdot\|\mid\|$ são equivalentes. Como $\|x\| \leq\|x\|$, para cada $x \in E$, a aplicação identidade $i d:(E, \||\cdot|||) \rightarrow(E,\|\cdot\|)$ é contínua. Logo, como mostramos que $(E,|\|\cdot\||)$ é espaço de Banach , pelo lema 1.20 podemos concluir que as normas $\|\cdot\|$ e || $\cdot \|$ são equivalentes.

A seguir estudamos uma importante propriedade topológica dos espaços de Banach com FDD incondicional.

Proposição 1.21. Seja E um espaço de Banach com FDD incondicional $\left\{E_{n}\right\}_{n \in \mathbb{N}}$. Então E é separável.

Lema 1.22. Seja X um espaço normado qualquer. Se existe um conjunto enumerável $A \subset X$ satisfazendo $\overline{[A]}=X$, então $X$ é separável.

\section{Demonstração (do lema):}

$X$ é um $\mathbb{K}$-espaço normado, com $\mathbb{K}=\mathbb{R}$ ou $\mathbb{C}$. Se $\mathbb{K}=\mathbb{R}$, definimos $Q \doteq \mathbb{Q}$, e se $\mathbb{K}=\mathbb{C}$, definimos $Q \doteq\{a+b i: a, b \in \mathbb{Q}\}$. Em ambos os casos, $Q$ é denso e enumerável em $\mathbb{K}$.

Vamos escrever $A=\left\{a_{n}\right\}_{n \in \mathbb{N}}$, e vamos definir

$$
D_{j} \doteq\left\{\sum_{n=1}^{j} q_{n} a_{n}: q_{1}, \ldots, q_{j} \in Q\right\} \text { e } D \doteq \cup_{j=1}^{\infty} D_{j}
$$


Cada $D_{j}$ é enumerável e portanto $D$ é enumerável. Vamos verificar que $D$ é denso em $X$, concluindo assim a demonstração.

Sejam $x \in X$ e $\epsilon>0$. Como $\overline{[A]}=X$, existem $m \in \mathbb{N}$ e $r_{1}, \ldots, r_{m} \in \mathbb{K}$ tais que

$$
\left\|x-\sum_{n=1}^{m} r_{n} a_{n}\right\| \leq \frac{\epsilon}{2}
$$

Sendo $Q$ denso em $\mathbb{K}$, existem $q_{1}, \ldots, q_{m} \in Q$ tais que, para cada $n \in\{1, \ldots, m\}$ temos $\left|r_{n}-q_{n}\right| \leq \frac{\epsilon}{2 m\left\|a_{n}\right\|}$ se $a_{n} \neq 0$ e $\left|r_{n}-q_{n}\right| \leq \frac{\epsilon}{2 m}$ se $a_{n}=0$. Então

$$
\begin{aligned}
\left\|x-\sum_{n=1}^{m} q_{n} a_{n}\right\| & \leq\left\|x-\sum_{n=1}^{m} r_{n} a_{n}\right\|+\left\|\sum_{n=1}^{m}\left(r_{n}-q_{n}\right) a_{n}\right\| \\
& \leq \frac{\epsilon}{2}+\sum_{n=1}^{m}\left|r_{n}-q_{n}\right|\left\|a_{n}\right\| \leq \frac{\epsilon}{2}+\frac{\epsilon}{2}=\epsilon,
\end{aligned}
$$

como queríamos.

\section{Demonstração (da proposição):}

Para cada $i \in \mathbb{N}$, seja $\left\{e_{i 1}, \ldots, e_{i n_{i}}\right\}$ uma base para $E_{i}$. O conjunto $B \doteq \cup_{i=1}^{\infty}\left\{e_{i 1}, \ldots, e_{i n_{i}}\right\}$ é enumerável, por ser reunião enumerável de conjuntos finitos; provemos que $\overline{[B]}=E$, e poderemos concluir pelo lema 1.22 que $E$ é separável. Sejam então $x \in E$ e $\epsilon>0$ quaisquer e mostremos que existe um elemento $y \in[B]$ tal que $\|x-y\|<\epsilon$.

$x$ se escreve na forma $x=\sum_{i=1}^{\infty} x_{i}$, onde para cada $i \in \mathbb{N}, x_{i}=\alpha_{i 1} e_{i 1}+\cdots+\alpha_{i n_{i}} e_{i n_{i}} \in E_{i}$ e existe $N \in \mathbb{N}$ tal que $\left\|x-\sum_{i=1}^{N} x_{i}\right\|<\epsilon$.

Mas $y \doteq \sum_{i=1}^{N} x_{i}=\sum_{i=1}^{N} \alpha_{i 1} e_{i 1}+\cdots+\alpha_{i n_{i}} e_{i n_{i}} \in[B]$, de onde segue o resultado.

Observação: Em [7] há um exemplo de espaço de Banach que é separável mas não possui FDD incondicional. Esse exemplo é conhecido como exemplo de Enflo.

Como consequência do lema, temos o seguinte:

Corolário 1.23. Seja E um espaço de Banach com base de Schauder. Então E é separável. 


\subsection{Topologia fraca}

Vamos definir em um espaço normado a topologia fraca. Para isto necessitaremos dos conceitos que a seguir.

Definição 1.24. Seja $X$ um espaço normado. Sejam $a \in X, f_{1}, \ldots, f_{n} \in X^{\prime}$ e $\epsilon>0$. Definimos

$$
U\left(a ; f_{1}, \ldots, f_{n} ; \epsilon\right) \doteq\left\{x \in X: \sup _{i=1 \ldots, n}\left|f_{i}(x-a)\right|<\epsilon\right\} .
$$

Diremos que $U\left(a ; f_{1}, \ldots, f_{n} ; \epsilon\right)$ é uma vizinhança fraca de a.

Diremos que $V \subset X$ é um conjunto fracamente aberto de $X$ se para cada $a \in V$ existirem $f_{1}, \ldots, f_{n} \in X^{\prime}$ e $\epsilon>0$ tais que $U\left(a ; f_{1}, \ldots, f_{n} ; \epsilon\right) \subset V$.

Observe que trivialmente $\emptyset$ é fracamente aberto.

Proposição 1.25. O conjunto

$$
\sigma\left(X, X^{\prime}\right) \doteq\{V \subset X: V \text { é fracamente aberto }\}
$$

é uma topologia sobre $X$.

$\sigma\left(X, X^{\prime}\right)$ é chamada de topologia fraca sobre $X$.

\section{Demonstração:}

1) Trivialmente se verifica que $\emptyset, X \subset \sigma\left(X, X^{\prime}\right)$.

2) Sejam $I$ um conjunto de índices não vazio e $\left\{V_{i}\right\}_{i \in I}$ tais que $V_{i} \in \sigma\left(X, X^{\prime}\right), \forall i \in I$. Vamos mostrar que $\cup_{i \in I} V_{i} \in \sigma\left(X, X^{\prime}\right)$.

Se $V_{i}=\emptyset, \forall i \in I$, então $\cup_{i \in I} V_{i}=\emptyset \in \sigma\left(X, X^{\prime}\right)$. Caso contrário, seja $a \in \cup_{i \in I} V_{i}$. Então existe $i_{o} \in I, a \in V_{i_{o}}$. Como $V_{i_{o}} \in \sigma\left(X, X^{\prime}\right)$, existem $f_{1}, \ldots, f_{n} \in X^{\prime}$ e $\epsilon>0$ tais que $U\left(a ; f_{1}, \ldots, f_{n} ; \epsilon\right) \subset V_{i_{o}} \subset \cup_{i \in I} V_{i}$ e portanto $\cup_{i \in I} V_{i} \in \sigma\left(X, X^{\prime}\right)$.

3) Sejam $V_{1}, V_{2} \in \sigma\left(X, X^{\prime}\right)$ e vamos mostrar que $V_{1} \cap V_{2} \in \sigma\left(X, X^{\prime}\right)$.

Se $V_{1} \cap V_{2}=\emptyset$, sabemos que $V_{1} \cap V_{2} \in \sigma\left(X, X^{\prime}\right)$. Suponhamos então que $V_{1} \cap V_{2} \neq \emptyset$, e seja $a \in V_{1} \cap V_{2}$.

Como $V_{1}, V_{2} \in \sigma\left(X, X^{\prime}\right)$, existem $f_{1}, \ldots, f_{n}, g_{1}, \ldots, g_{m} \in X^{\prime}$ e $\epsilon_{1}, \epsilon_{2}>0$ tais que

$$
U\left(a ; f_{1}, \ldots, f_{n} ; \epsilon_{1}\right) \subset V_{1} \text { e } U\left(a ; g_{1}, \ldots, g_{m} ; \epsilon_{2}\right) \subset V_{2}
$$


Vamos mostrar que $U\left(a ; f_{1}, \ldots, f_{n}, g_{1}, \ldots, g_{m} ; \epsilon\right) \subset V_{1} \cap V_{2}$, onde $\epsilon \doteq \min \left\{\epsilon_{1}, \epsilon_{2}\right\}$. De fato, se $x \in U\left(a ; f_{1}, \ldots, f_{n}, g_{1}, \ldots, g_{m} ; \epsilon\right)$, então

$$
\sup \left\{\left|f_{1}(x-a)\right|, \ldots\left|f_{n}(x-a)\right|,\left|g_{1}(x-a)\right|, \ldots,\left|g_{m}(x-a)\right|\right\}<\epsilon \leq \epsilon_{1}
$$

Em particular, $\left|f_{i}(x-a)\right|<\epsilon_{1}, \forall i \in\{1, \ldots, n\}$ e assim temos que $x \in V_{1}$.

De forma análoga provamos que $x \in V_{2}$. Então $U\left(a ; f_{1}, \ldots, f_{n}, g_{1}, \ldots, g_{m} ; \epsilon\right) \subset V_{1} \cap V_{2}$.

Proposição 1.26. Seja $X$ um espaço normado, e seja $\tau(X)$ a topologia induzida pela norma de $X$. Então

$$
\sigma\left(X, X^{\prime}\right) \subset \tau(X)
$$

\section{Demonstração:}

Seja $V \in \sigma\left(X, X^{\prime}\right)$ não vazio, e vamos mostrar que $V \in \tau(X)$. Fixado $a \in V$, basta verificar que existe $r>0$ tal que $B_{r}(a) \subset V$.

Como $V \in \sigma\left(X, X^{\prime}\right)$, existem $f_{1}, \ldots, f_{n} \in X^{\prime}$ e $\epsilon>0$ tais que $U\left(a ; f_{1}, \ldots, f_{n} ; \epsilon\right) \subset V$.

Seja $r=\frac{\epsilon}{\max _{i=1, \ldots, n}\left\{\left\|f_{i}\right\|\right\}+1}$, e vamos provar que $B_{r}(a) \subset U\left(a ; f_{1}, \ldots, f_{n} ; \epsilon\right)$.

Seja $x \in B_{r}(a)$. Então $\|x-a\|<\frac{\epsilon}{\max _{i=1, \ldots, n}\left\{\left\|f_{i}\right\|\right\}+1}$, e desta forma para cada $i \in$ $\{1, \ldots, n\}$,

$$
\left|f_{i}(x-a)\right| \leq\left\|f_{i}\right\|\|x-a\|<\left\|f_{i}\right\| \frac{\epsilon}{\max _{i=1, \ldots, n}\left\{\left\|f_{i}\right\|\right\}+1}<\epsilon
$$

e portanto $\sup _{i=1, \ldots, n}\left\{\left|f_{i}(x-a)\right|\right\}<\epsilon$, de forma que $x \in U\left(a ; f_{1}, \ldots, f_{n} ; \epsilon\right)$, como queríamos.

Precisamos estabelecer a noção de convergência fraca de sequências para podermos posteriormente definir o que é continuidade sequencial fraca:

Definição 1.27. Seja $X$ um espaço normado. Uma sequência $\left(x_{n}\right)_{n}$ é dita fracamente convergente (para $x \in X$ ) se ela é convergente (para $x$ ) com relação à topologia fraca sobre $X$.

Neste caso, escreveremos $x_{n} \stackrel{w}{\rightarrow} x$.

A seguinte caracterização de convergência fraca será muito utilizada quando estivermos trabalhando com polinômios: 
Teorema 1.28. Sejam $X$ um espaço normado, $x \in X$ e $\left(x_{n}\right)_{n}$ uma sequência em $X$. Então $x_{n} \stackrel{w}{\rightarrow} x$ se e somente se $f\left(x_{n}\right) \rightarrow f(x), \forall f \in X^{\prime}$.

\section{Demonstração:}

Suponhamos que $x_{n} \stackrel{w}{\rightarrow} x$ e seja $f \in X^{\prime}$. Dado $\epsilon>0$, existe $n_{o} \in \mathbb{N}$ tal que para cada $n \geq n_{o}$ natural, $x_{n} \in U(x ; f ; \epsilon)$, pelo fato deste conjunto ser fracamente aberto.

Assim, para $n \geq n_{o}$ natural, $\left|f(x)-f\left(x_{n}\right)\right|<\epsilon$. Como $\epsilon>0$ é arbitrário, temos que $f\left(x_{n}\right) \rightarrow f(x)$, como queríamos.

Suponhamos agora que $f\left(x_{n}\right) \rightarrow f(x)$ para cada $f \in X^{\prime}$. Seja $V$ um conjunto fracamente aberto contendo $x$. Então existem $f_{1}, \ldots, f_{k} \in X^{\prime}$ e $\epsilon>0$ tais que

$$
x \in U\left(x ; f_{1}, \ldots, f_{k} ; \epsilon\right) \subset V .
$$

Para cada $i \in\{1, \ldots, k\}$, pelo fato de $f_{i}\left(x_{n}\right) \rightarrow f_{i}(x)$, temos que existe $n_{i} \in \mathbb{N}$ tal que $\left|f_{i}\left(x_{n}\right)-f_{i}(x)\right|<\epsilon$ para cada $n \geq n_{i}$ natural.

Se tomarmos $n_{o} \doteq \max _{i=1, \ldots, k}\left\{n_{i}\right\}$, teremos para cada $n \geq n_{o}$ natural e cada $i \in$ $\{1, \ldots, k\}$ que $\left|f_{i}\left(x_{n}\right)-f_{i}(x)\right|<\epsilon$ e portanto $x_{n} \in U\left(x ; f_{1}, \ldots, f_{k} ; \epsilon\right) \subset V$. Ou seja, $\left(x_{n}\right)_{n}$ converge fracamente a $x$.

Corolário 1.29. Sejam $X$ e $Y$ espaços normados, $T \in \mathcal{L}(X ; Y)$ e $\left(x_{n}\right)_{n}$ uma sequência em $X$ convergindo fracamente a $x$. Então $T\left(x_{n}\right) \stackrel{w}{\rightarrow} T(x)$.

\section{Demonstração:}

Basta verificar que, dado $f \in Y^{\prime}, f\left(T\left(x_{n}\right)\right) \rightarrow f((T(x))$. Mas, já que $T$ é contínua, $f \circ T \in X^{\prime}$, e então podemos usar o Teorema acima para concluir a demonstração.

Corolário 1.30. Sejam $X$ um espaço normado, $x \in X$ e $\left(x_{n}\right)_{n}$ uma sequência em $X$. Se $x_{n} \rightarrow x$, então $x_{n} \stackrel{w}{\rightarrow} x$.

\section{Demonstração:}

Se $x_{n} \rightarrow x$, temos que $f\left(x_{n}\right) \rightarrow f(x), \forall f \in X^{\prime}$, e pelo Teorema anterior podemos concluir que $x_{n} \stackrel{w}{\rightarrow} x$.

Exemplo 1.31. No espaço $l_{p}, 1<p<\infty$, a sequência $\left(e^{k}\right)_{k}$ formada pelos elementos da base canônica de $l_{p}$ é tal que $e^{k} \stackrel{w}{\rightarrow} 0$ mas $\left(e^{k}\right)_{k}$ não converge em $l_{p}$. 
A sequência $\left(e^{k}\right)_{k}$ não converge pois para cada $l, k$ naturais distintos temos que $\| e^{l}-$ $e^{k} \|=\sqrt[p]{2}$, e assim $\left(e^{k}\right)_{k}$ não é de Cauchy.

Para mostrar que $e^{k} \stackrel{w}{\rightarrow} 0$, pelo Teorema anterior basta que, para cada $f \in l_{p}^{\prime}, f\left(e^{k}\right) \rightarrow$ $f(0)=0$.

Fixo $f \in l_{p}^{\prime}$. Então existe $\left(a_{n}\right)_{n} \in l_{q}$, onde $\frac{1}{p}+\frac{1}{q}=1$, tal que $f(x)=\sum_{n=1}^{\infty} a_{n} x_{n}$, para cada $x \in l_{p}$. Logo, $f\left(e^{k}\right)=a_{k}$, e $a_{k} \rightarrow 0$ pelo fato de $\left(a_{n}\right)_{n} \in l_{q}$ e assim concluimos nossa verificação.

A seguir temos duas importantes propriedades das sequências fracamente convergentes:

Teorema 1.32. Sejam $X$ um espaço normado, $x \in X$ e $\left(x_{n}\right)_{n}$ uma sequência em $X$ convergindo fracamente a $x$. Então as seguintes afirmações são verdadeiras:

1) $\left(x_{n}\right)_{n}$ é limitada;

2) O limite de $\left(x_{n}\right)_{n}$ com relação à topologia fraca é único.

Demontração:

1) Definimos para cada $n \in \mathbb{N} F_{n} \in X^{\prime \prime}$ por $F_{n}(f) \doteq f\left(x_{n}\right)$, para cada $f \in X^{\prime}$. Como $x_{n} \stackrel{w}{\rightarrow} x$, para cada $f \in X^{\prime}$ temos que $f\left(x_{n}\right) \rightarrow f(x)$, e assim

$$
\lim _{n \rightarrow \infty} F_{n}(f)=\lim _{n \rightarrow \infty} f\left(x_{n}\right)=f(x)
$$

de forma que para cada $f \in X^{\prime}$ o conjunto $\left\{F_{n}(f): n \in \mathbb{N}\right\}$ é limitado. Como além disso $X^{\prime}$ é um espaço de Banach, podemos usar o princípio da limitação uniforme (1.6) para concluir que existe $M>0$ tal que $\left\|F_{n}\right\| \leq M$, para cada $n \in \mathbb{N}$. Assim,

$$
\left|F_{n}(f)\right| \leq\left\|F_{n}\right\|\|f\| \leq M\|f\|, \forall f \in X^{\prime}, n \in \mathbb{N} .
$$

Vamos supor que $\left\|x_{n}\right\|>0$, para cada $n \in \mathbb{N}$. Pelo corolário 1.3 (do Teorema de Hahnbanach) podemos afirmar que para cada $n \in \mathbb{N}$ existe $f_{n} \in X^{\prime}$ tal que $f_{n}\left(x_{n}\right)=\left\|x_{n}\right\|$ e $\left\|f_{n}\right\|=1$. Então para cada $n \in \mathbb{N}$,

$$
\left\|x_{n}\right\|=\left|f_{n}\left(x_{n}\right)\right|=\left|F_{n}\left(f_{n}\right)\right| \leq M\left\|f_{n}\right\|=M,
$$

ou seja, $\left(x_{n}\right)_{n}$ é limitada. Observe que a desigualdade acima vale trivialmente quando $\left\|x_{n}\right\|=0$.

2) Suponhamos que $x_{n} \stackrel{w}{\rightarrow} y$, para algum $y \in X$ distinto de $x$. Como já sabemos que $x_{n} \stackrel{w}{\rightarrow} x$, temos que para cada $f \in X^{\prime}, f\left(x_{n}\right) \rightarrow f(x)$ e $f\left(x_{n}\right) \rightarrow f(y)$. Como o limite em 
$\mathbb{K}$ é único, concluimos que $f(x)=f(y)$, para cada $f \in X^{\prime}$. Agora, pelo corolário 1.4 (do Teorema de Hahn-Banach) existe $f_{0} \in X^{\prime}$ tal que $f_{0}(x) \neq f_{0}(y)$, uma contradição.

Proposição 1.33. Sejam $X$ um espaço normado, $x \in X$ e $\left(x_{n}\right)_{n}$ uma sequência em $X$. Então $x_{n} \stackrel{w}{\rightarrow} x$ se e somente se as seguintes duas afirmações são verdadeiras:

1) A sequência $\left(\left\|x_{n}\right\|\right)_{n}$ é limitada em $\mathbb{K}$;

2) Existe um conjunto $M \subset X^{\prime}$ tal que $[M]$ é denso em $X^{\prime}$ e $\forall f \in M, f\left(x_{n}\right) \rightarrow f(x)$.

\section{Demonstração:}

Se $x_{n} \stackrel{w}{\rightarrow} x$, as afirmações (1) e (2) seguem diretamente dos Teoremas 1.32 e 1.28 , respectivamente.

Suponhamos agora que $\left(\left\|x_{n}\right\|\right)_{n}$ é limitada e que exista $M \subset X^{\prime}$ satisfazendo as condições de (2). Vamos mostrar que, dado $f \in X^{\prime}, f\left(x_{n}\right) \rightarrow f(x)$.

Seja $\epsilon>0$, e seja $K>0$ tal que $\left\|x_{n}\right\|<K, \forall n \in \mathbb{N}$ e $\|x\|<K$. Como $[M]$ é denso em $X^{\prime}$, existe $g \in M$ tal que $\|g-f\|<\frac{\epsilon}{3 K}$. Como por hipótese $g\left(x_{n}\right) \rightarrow g(x)$, existe $n_{o} \in \mathbb{N}$ tal que para cada $n \geq n_{o}$ natural temos que $\left|g\left(x_{n}\right)-g(x)\right|<\frac{\epsilon}{3}$.

Então, para cada natural $n \geq n_{o}$

$$
\begin{aligned}
\left|f\left(x_{n}\right)-f(x)\right| & \leq\left|f\left(x_{n}\right)-g\left(x_{n}\right)\right|+\left|g\left(x_{n}\right)-g(x)\right|+|g(x)-f(x)| \\
& <\frac{\epsilon}{3 K}\left\|x_{n}\right\|+\frac{\epsilon}{3}+\frac{\epsilon}{3 K}\|x\| \\
& \leq \frac{\epsilon}{3}+\frac{\epsilon}{3}+\frac{\epsilon}{3}=\epsilon
\end{aligned}
$$

e assim podemos concluir que $f\left(x_{n}\right) \rightarrow f(x)$.

O corolário 1.34 nos dá mais explicitamente uma caracterização das sequências fracamente convergentes nos espaços $l_{p}$, com $1<p<\infty$.

Corolário 1.34. Considero o espaço $l_{p}$, com $1<p<\infty$, e sejam $x=\left(x_{1}, x_{2}, \ldots\right)$ um elemento e $\left(x^{k}\right)_{k}$ uma sequência em $l_{p}$, onde cada $x^{k}=\left(x_{1}^{k}, x_{2}^{k}, \ldots\right)$.

Vale então que $x_{k} \stackrel{w}{\rightarrow} x$ se e somente se as seguintes duas afirmações são verdadeiras:

1) A sequência $\left(\left\|x^{k}\right\|\right)_{k}$ é limitada em $\mathbb{K}$;

2) Para cada $n \in \mathbb{N}, x_{n}^{k} \stackrel{k}{\rightarrow} x_{n}$.

Observe que a sequência $\left(k e^{k}\right)_{k}$ em $l_{p}$ é um exemplo de sequência que satisfaz a condição (2) mas não satisfaz a condição (1) do corolário. 


\section{Demonstração:}

Basta tomar $M=\left\{e^{j}\right\}_{j} \subset l_{q}=l_{p}^{\prime}$, com $\frac{1}{p}+\frac{1}{q}=1$, que satisfaz $\overline{\left[\left\{e^{j}\right\}_{j}\right]}=l_{q}$. Para este $M$, a condição (2) da proposição acima é equivalente à condição (2) do corolário, pois para cada $j \in \mathbb{N}$, $e^{j}$ é um representante do funcional $f_{j}:\left(x_{n}\right)_{n} \mapsto x_{j}$. 


\section{Capítulo 2}

\section{Polinômios homogêneos contínuos}

\subsection{Aplicações multilineares}

A definição de polinômio homogêneo em um espaço de Banach é baseada na definição de aplicações multilineares. Esses dois tópicos são apresentados neste capítulo de forma bastante suscinta, de forma que apresentam-se apenas os resultados que serão utilizados no próximo capítulo. Um estudo com mais detalhes pode ser encontrado em [16] e [19].

Definição 2.1. Sejam $m \in \mathbb{N}$ e $X_{1}, \ldots, X_{m}, Y \mathbb{K}$-espaços vetoriais. Uma aplicação $A$ : $X_{1} \times \cdots \times X_{m} \rightarrow Y$ é dita uma aplicação $\boldsymbol{m}$-linear se para cada $i \in\{1, \ldots, m\}$ a aplicação $x_{i} \mapsto A\left(x_{1}, \ldots, x_{i}, \ldots, x_{m}\right)$ for linear de $X_{i}$ em $Y$.

Definimos também $\mathcal{L}_{a}\left(X_{1}, \ldots, X_{m} ; Y\right)$ como sendo o $\mathbb{K}$-espaço vetorial de todas as aplicações m-lineares de $X_{1} \times \cdots \times X_{m}$ em $Y$.

Quando $X=X_{1}=\cdots=X_{m}$, escrevemos $\mathcal{L}_{a}\left({ }^{m} X ; Y\right) \doteq \mathcal{L}_{a}\left(X_{1}, \ldots, X_{m} ; Y\right)$. Por conveniência definimos $\mathcal{L}_{a}\left({ }^{0} X ; Y\right) \doteq Y$.

Quando $Y=\mathbb{K}$, escrevemos $\mathcal{L}_{a}\left({ }^{m} X\right) \doteq \mathcal{L}_{a}\left({ }^{m} X ; \mathbb{K}\right)$.

Para o estudo da continuidade das aplicações m-lineares, estaremos sempre considerando espaços normados, e consideramos em $X_{1} \times \cdots \times X_{m}$ a norma $\|\cdot\|: X_{1} \times \cdots \times X_{m} \rightarrow \mathbb{R}$ definida por $\left\|\left(x_{1}, \ldots, x_{m}\right)\right\| \doteq \sup _{i \in\{1, \ldots, m\}}\left\|x_{i}\right\|$.

Uma caracterização de continuidade de transformações m-lineares se dá pelo seguinte Teorema: 
Teorema 2.2. Sejam $X_{1}, \ldots, X_{m}, Y$ espaços normados e $A \in \mathcal{L}_{a}\left(X_{1}, \ldots, X_{m} ; Y\right)$. Então são equivalentes as seguintes afirmações:

1) A é contínua;

2) A é contínua na origem;

3) Existe $M>0$ tal que $\left\|A\left(x_{1}, \cdots, x_{m}\right)\right\| \leq M\left\|x_{1}\right\| \ldots\left\|x_{m}\right\|$, para cada $\left(x_{1}, \ldots, x_{m}\right) \in$ $X_{1} \times \cdots \times X_{m}$.

\section{Demonstração:}

É evidente que $(1) \Rightarrow(2)$.

Para mostrar que $(2) \Rightarrow(3)$ temos por hipótese $A$ contínua na origem. Logo, existe $\delta>0$ tal que se $\|x\|<\delta$ então $\|A(x)\| \leq 1$. Seja $x=\left(x_{1}, \ldots, x_{n}\right)$ com $x_{i} \neq 0$ para cada $i \in$ $\{1, \ldots, m\}$. Temos que $\left\|\left(\frac{\delta x_{1}}{2\left\|x_{1}\right\|}, \ldots, \frac{\delta x_{n}}{2\left\|x_{n}\right\|}\right)\right\|<\delta, \operatorname{assim}\left\|A\left(\frac{\delta x_{1}}{2\left\|x_{1}\right\|}, \ldots, \frac{\delta x_{n}}{2\left\|x_{n}\right\|}\right)\right\| \leq 1$. Desta forma, $\left\|A\left(x_{1}, \ldots, x_{n}\right)\right\| \leq \frac{2^{m}}{\delta^{m}}\left\|x_{1}\right\| \ldots\left\|x_{m}\right\|$. Se para algum $i \in\{1, \ldots, m\}$ tivermos que $x_{i}=0$, a desigualdade acima também é verdadeira, e portanto a afirmação (3) é verdadeira.

Vamos mostrar que $(3) \Rightarrow(1)$.

Observe que $A(x)-A(y)=A\left(x_{1}-y_{1}, \ldots, x_{m}\right)+\ldots+A\left(y_{1}, \ldots, y_{m-1}, x_{m}-y_{m}\right)$. Desta forma,

$$
\begin{aligned}
\|A(x)-A(y)\| & \leq\left\|A\left(x_{1}-y_{1}, \ldots, x_{m}\right)\right\|+\ldots+\left\|A\left(y_{1}, \ldots, y_{m-1}, x_{m}-y_{m}\right)\right\| \\
& \leq M\left(\left\|x_{1}-y_{1}\right\|\left\|x_{2}\right\| \ldots\left\|x_{m}\right\|+\cdots+\left\|y_{1}\right\| \ldots\left\|y_{m-1}\right\|\left\|x_{m}-y_{m}\right\|\right) .
\end{aligned}
$$

Vamos agora mostrar que, fixado $r>0 A$ é uniformemente contínua em $B_{r}(0) \doteq\{x \in$ $\left.X_{1} \times \cdots \times X_{m}:\|x\|<r\right\}$.

Sejam $x, y \in X$ com $\|x\|,\|y\|<r$, então para cada $i \in\{1, \ldots, m\}$ temos que $\left\|x_{i}\right\|<r$ e $\left\|y_{i}\right\|<r$. Logo,

$$
\|A(x)-A(y)\| \leq M r^{m-1}\left(\left\|x_{1}-y_{1}\right\|+\cdots+\left\|x_{m}-y_{m}\right\|\right) \leq M r^{m-1} m\|x-y\|
$$

e isto nos permite conluir que $A$ é uniformemente contínua sobre $B_{r}(0)$. Como $r$ é arbitrário, $A$ é contínua.

Vamos destacar o seguinte detalhe da demontração de $(3) \Rightarrow(1)$ :

Corolário 2.3. Sejam $X$ e $Y$ espaços normados, $U$ um subconjunto limitado de $X \times$ $\cdots \times X$ e $A \in \mathcal{L}_{a}\left({ }^{m} X ; Y\right)$ contínua. Então existe uma constante $M \geq 0$ tal que para cada 
$x, y \in U$,

$$
\|A(x)-A(y)\| \leq M\|x-y\|
$$

e por conseguinte $A$ é uniformemente contínua sobre $U$.

Observação: Sabemos que as transformações lineares contínuas são uniformemente contínuas em todo o seu domínio. Este resultado não é verdadeiro para transformações multilineares quaisquer, na realidade qualquer aplicação $m$-linear com $m>1$ não nula não é uniformemente contínua em todo o domínio.

Para verificar esse fato, seja $A \in \mathcal{L}_{a}\left(X_{1}, \ldots, X_{m} ; Y\right)$ contínua não nula. Então existem $\epsilon>0$ e $\left(x_{1}, \ldots, x_{m}\right) \in X_{1} \times \cdots \times X_{m}$ tais que $\left\|A\left(x_{1}, \ldots, x_{m}\right)\right\|>\epsilon>0$. Para cada $\delta>0$, podemos escolher $\lambda \in \mathbb{K}$ tal que $\left\|\lambda x_{1}\right\|<\delta$. Mas então temos que

$$
\left\|\left(x_{1}+\lambda x_{1}, \frac{x_{2}}{\lambda}, x_{3}, \ldots, x_{m}\right)-\left(x_{1}, \frac{x_{2}}{\lambda}, x_{3}, \ldots, x_{m}\right)\right\|=\left\|\lambda x_{1}\right\|<\delta
$$

e

$$
\begin{aligned}
\left\|A\left(x_{1}+\lambda x_{1}, \frac{x_{2}}{\lambda}, x_{3}, \ldots, x_{m}\right)-A\left(x_{1}, \frac{x_{2}}{\lambda}, x_{3}, \ldots, x_{m}\right)\right\| & =\left\|A\left(\lambda x_{1}, \frac{x_{2}}{\lambda}, x_{3}, \ldots, x_{m}\right)\right\| \\
& =\left\|A\left(x_{1}, x_{2}, \ldots, x_{m}\right)\right\|>\epsilon .
\end{aligned}
$$

Logo, $A$ não é uniformemente contínua.

Definição 2.4. Sejam $X_{1}, \ldots, X_{m}, Y$ espaços normados. Denotamos por $\mathcal{L}\left(X_{1}, \ldots, X_{m} ; Y\right)$ o subespaço formado pelas transformações multilineares continuas de $\mathcal{L}_{a}\left(X_{1}, \ldots, X_{m} ; Y\right)$. Quando $X_{1}=\cdots=X_{m}=X$, escrevemos $\mathcal{L}\left({ }^{m} X ; Y\right)=\mathcal{L}(X, \ldots, X ; Y)$ e quando $Y=\mathbb{K}$ escrevemos $\mathcal{L}\left({ }^{m} X\right) \doteq \mathcal{L}\left({ }^{m} X ; \mathbb{K}\right)$.

O espaço $\mathcal{L}\left(X_{1}, \ldots, X_{m} ; Y\right)$ é um espaço normado se munido da norma dada pela seguinte proposição:

Proposição 2.5. $\|\cdot\|: \mathcal{L}\left(X_{1}, \ldots, X_{m} ; Y\right) \rightarrow \mathbb{R}$ definida por

$$
\|A\| \doteq \sup \left\{\left\|A\left(x_{1}, \ldots, x_{m}\right)\right\|:\left(x_{1}, \ldots, x_{m}\right) \in X_{1} \times \cdots \times X_{m},\left\|\left(x_{1}, \ldots, x_{m}\right)\right\| \leq 1\right\}
$$

é uma norma em $\mathcal{L}\left(X_{1}, \ldots, X_{m} ; Y\right)$, e para cada $A \in \mathcal{L}\left(X_{1}, \ldots, X_{m} ; Y\right)$,

$$
\|A\|=\inf \left\{M \geq 0:\left\|A\left(x_{1}, \ldots, x_{m}\right)\right\| \leq M\left\|x_{1}\right\| \ldots\left\|x_{m}\right\|, \forall\left(x_{1}, \ldots, x_{m}\right) \in X_{1} \times \cdots \times X_{m}\right\} .
$$




\section{Demonstração:}

Observemos inicialmente que para cada $A \in \mathcal{L}\left(X_{1}, \ldots, X_{m} ; Y\right)$ temos que

$0 \leq \sup \left\{\left\|A\left(x_{1}, \ldots, x_{m}\right)\right\|:\left(x_{1}, \ldots, x_{m}\right) \in X_{1} \times \cdots \times X_{m},\left\|\left(x_{1}, \ldots, x_{m}\right)\right\| \leq 1\right\}<\infty$,

pois como já vimos $A$ leva conjuntos limitados em conjuntos limitados e portanto $\|\cdot\|$ está bem definida.

Vamos verificar que $\|A\|=0 \Leftrightarrow A=0$. A implicação à esquerda é trivial. Para mostrarmos a outra implicação seja $A \in \mathcal{L}\left(X_{1}, \ldots, X_{m} ; Y\right)$ tal que $\|A\|=0$ e $x=$ $\left(x_{1}, \ldots, x_{m}\right) \in X_{1} \times \cdots \times X_{m}$ qualquer, e verifiquemos que $A(x)=0$.

Se $x_{i}=0$ para algum $i \in\{1, \ldots, m\}$, evidentemente $A(x)=0$.

Se $x_{i} \neq 0$ para cada $i \in\{1, \ldots, m\}$, temos que $\left\|\left(\frac{x_{1}}{\left\|x_{1}\right\|}, \ldots, \frac{x_{m}}{\left\|x_{m}\right\|}\right)\right\|=1$, e logo como $\|A\|=0$ temos $A\left(\frac{x_{1}}{\left\|x_{1}\right\|}, \ldots, \frac{x_{m}}{\left\|x_{m}\right\|}\right)=0$.

Assim,

$$
\|A(x)\|=\left\|A\left(\frac{x_{1}}{\left\|x_{1}\right\|}, \ldots, \frac{x_{m}}{\left\|x_{m}\right\|}\right)\right\|\left\|x_{1}\right\| \ldots\left\|x_{m}\right\|=0
$$

e podemos concluir que $A=0$.

Para cada $\lambda \in \mathbb{K}$

$$
\|\lambda A\|=\sup \{\|\lambda A(x)\|:\|x\| \leq 1\}=|\lambda| \sup \{\|A(x)\|:\|x\| \leq 1\}=\mid \lambda\|A\| .
$$

Falta verificar a desigualdade triangular. Sejam $A$ e $B \in \mathcal{L}\left(X_{1}, \ldots, X_{m} ; Y\right)$. Para cada $x=\left(x_{1}, \ldots, x_{m}\right) \in X_{1} \times \cdots \times X_{m}$ de norma menor ou igual a 1 temos que

$$
\|A(x)+B(x)\| \leq\|A(x)\|+\|B(x)\| \leq\|A\|+\|B\|
$$

e portanto

$$
\|A+B\|=\sup \left\{\|A(x)+B(x)\|: x \in X_{1} \times \cdots \times X_{m},\|x\| \leq 1\right\} \leq\|A\|+\|B\| .
$$

Vamos verificar agora que para cada $A \in \mathcal{L}\left(X_{1}, \ldots, X_{m} ; Y\right),\|A\|=\inf \{M>0$ : $\left.\left\|A\left(x_{1}, \ldots, x_{m}\right)\right\| \leq M\left\|x_{1}\right\| \ldots\left\|x_{m}\right\|, \forall\left(x_{1}, \ldots, x_{m}\right) \in X_{1} \times \cdots \times X_{m}\right\}$. Seja $M \geq 0$ tal que $\left\|A\left(x_{1}, \ldots, x_{m}\right)\right\| \leq M\left\|x_{1}\right\| \ldots\left\|x_{m}\right\|, \forall\left(x_{1}, \ldots, x_{m}\right) \in X_{1} \times \cdots \times X_{m}$. Então para cada $x=\left(x_{1}, \ldots, x_{m}\right) \in X_{1} \times \cdots \times X_{m}$ com $\|x\| \leq 1$ temos que $\|A(x)\| \leq M$, e assim $\|A\| \leq M$. Logo,

$\|A\| \leq \inf \left\{M \geq 0:\left\|A\left(x_{1}, \ldots, x_{m}\right)\right\| \leq M\left\|x_{1}\right\| \ldots\left\|x_{m}\right\|, \forall\left(x_{1}, \ldots, x_{m}\right) \in X_{1} \times \cdots \times X_{m}\right\}$. 
Mostremos agora que $\|A\|$ é a maior cota inferior para $\left\{M \geq 0:\left\|A\left(x_{1}, \ldots, x_{m}\right)\right\| \leq\right.$ $\left.M\left\|x_{1}\right\| \ldots\left\|x_{m}\right\|, \forall\left(x_{1}, \ldots, x_{m}\right) \in X_{1} \times \cdots \times X_{m}\right\}$. Para tal, vamos fixar $\epsilon>0$ qualquer e mostrar que $\|A\|+\epsilon$ não é cota inferior para $\left\{M \geq 0:\left\|A\left(x_{1}, \ldots, x_{m}\right)\right\| \leq\right.$ $\left.M\left\|x_{1}\right\| \ldots\left\|x_{m}\right\|, \forall\left(x_{1}, \ldots, x_{m}\right) \in X_{1} \times \cdots \times X_{m}\right\}$. Basta mostrar que $M \doteq\|A\|+\frac{\epsilon}{2}$ é tal que $\left\|A\left(x_{1}, \ldots, x_{m}\right)\right\| \leq\left(\|A\|+\frac{\epsilon}{2}\right)\left\|x_{1}\right\| \ldots\left\|x_{m}\right\|, \forall\left(x_{1}, \ldots, x_{m}\right) \in X_{1} \times \cdots \times X_{m}$. A desigualdade vale trivialmente se $\left\|x_{i}\right\|=0$ para algum $i \in\{1, \ldots, m\}$. Consideremos então $\left\|x_{1}\right\|, \ldots,\left\|x_{m}\right\|$ não nulos. Então $\left\|\left(\frac{x_{1}}{\left\|x_{1}\right\|}, \ldots, \frac{x_{m}}{\left\|x_{m}\right\|}\right)\right\|=1$, e pela definição de $\|A\|$ temos que

$$
\left\|A\left(\frac{x_{1}}{\left\|x_{1}\right\|}, \ldots, \frac{x_{m}}{\left\|x_{m}\right\|}\right)\right\| \leq\|A\|<\|A\|+\frac{\epsilon}{2}
$$

Logo,

$$
\left\|x_{1}\right\| \ldots\left\|x_{m}\right\|\left\|A\left(\frac{x_{1}}{\left\|x_{1}\right\|}, \ldots, \frac{x_{m}}{\left\|x_{m}\right\|}\right)\right\|=\left\|A\left(x_{1}, \ldots, x_{m}\right)\right\|<\left(\|A\|+\frac{\epsilon}{2}\right)\left\|x_{1}\right\| \ldots\left\|x_{m}\right\|,
$$

como queríamos.

Nem toda transformação m-linear contínua em cada variável é continua, de acordo com o seguinte exemplo:

Exemplo 2.6. Seja $E \doteq C_{L_{1}}[0,1]$. Então a transformação 2-linear $B(f, g) \doteq \int_{0}^{1} f(t) g(t) d t$ é contínua em cada variável mas não é contínua.

Se impusermos a condição adicional de os espaços de partida serem de Banach, a continuidade passa a ser equivalente à continuidade em cada variável, como está formalizado no Teorema abaixo:

Teorema 2.7. Sejam $E_{1}, \ldots, E_{m}$ espaços de Banach e $Y$ um espaço normado. Então $A \in \mathcal{L}_{a}\left(E_{1}, \ldots, E_{m} ; Y\right)$ é contínua se e somente se o é em cada variável.

\section{Demonstração:}

Suponhamos que $A$ é contínua. As funções $x_{i} \mapsto A\left(x_{1}, \ldots, x_{i}, \ldots, x_{m}\right)$ são contínuas pois são restrições de $A$ a $\left\{x_{1}\right\} \times \cdots \times\left\{x_{i-1}\right\} \times E_{i} \times\left\{x_{i}+1\right\} \times \cdots \times\left\{x_{m}\right\} \subset E_{1} \times \cdots \times E_{m}$.

Vamos mostrar a outra implicação primeiro no caso em que $A$ é bilinear. Seja $A$ : $E_{1} \times E_{2} \rightarrow Y$ bilinear contínua em cada variável. Para cada $y$ em $E_{2}$ vamos definir $A_{y}(x) \doteq A(x, y)$, uma aplicação linear contínua por hipótese. Para cada $x \in E_{1}, A_{x}(y) \doteq$ 
$A(x, y)$ também é linear e contínua, e portanto existe $M_{x}>0$ tal que $\left\|A_{x}(y)\right\| \leq M_{x}\|y\|$, para cada $y \in E_{2}$.

Temos que $\left\|A_{y}(x)\right\|=\|A(x, y)\|=\left\|A_{x}(y)\right\| \leq M_{x}\|y\|$. Em particular, se $y \in E_{2}$ é tal que $\|y\| \leq 1$, então $\left\|A_{y}(x)\right\| \leq M_{x}$.

Vamos considerar a família $\mathcal{F} \doteq\left\{A_{y}: y \in E_{2},\|y\| \leq 1\right\}$. Então $\left\|A_{y}(x)\right\| \leq M_{x}$, para cada $A_{y}$ em $\mathcal{F}$. Pelo princípio da limitação uniforme (1.6), existe $M>0$ tal que $\left\|A_{y}\right\| \leq M$, para cada $A_{y} \in \mathcal{F}$. Então para cada $(x, y) \in E_{1} \times E_{2}$ tal que $\|x\| \leq 1 \mathrm{e}$ $\|y\| \leq 1$, temos que $\|A(x, y)\|=\left\|A_{y}(x)\right\| \leq\left\|A_{y}\right\| \leq M$, e logo para cada $(x, y) \in E_{1} \times E_{2}$ temos $\|A(x, y)\| \leq M\|x\|\|y\|$. Então $A$ é contínua.

Vamos provar a tese por indução: suponhamos que todo operador $(m-1)$-linear contínuo em cada variável é contínuo. Então para cada $x_{m} \in E_{m}$ o operador $A_{x_{m}} \in$ $\mathcal{L}_{a}\left(E_{1} \times \cdots \times E_{m-1} ; Y\right)$ definido por $A_{x_{m}}\left(x_{1}, \ldots, x_{m-1}\right) \doteq A\left(x_{1}, \ldots, x_{m-1}, x_{m}\right)$ é contínuo em cada variável, portanto contínuo. Logo, existe $M_{x_{m}}$ tal que

$$
\left\|A\left(x_{1}, \ldots, x_{m}\right)\right\|=\left\|A_{x_{m}}\left(x_{1}, \ldots, x_{m-1}\right)\right\| \leq M_{x_{m}}\left\|x_{1}\right\| \ldots\left\|x_{m-1}\right\| .
$$

Se adicionalmente $\left\|x_{i}\right\| \leq 1$ para cada $i \in\{1, \ldots, m-1\}$ temos que $\left\|A\left(x_{1}, \ldots, x_{m-1}, x_{m}\right)\right\| \leq$ $M_{x_{m}}$. Agora vamos definir, para cada $\left(x_{1}, \ldots, x_{m-1}\right) \in E_{1} \times \cdots \times E_{m-1}$, o operador linear $A_{x_{1}, \ldots, x_{m-1}} \in \mathcal{L}\left(E_{m} ; Y\right)$ por $A_{x_{1}, \ldots, x_{m-1}}\left(x_{m}\right) \doteq A\left(x_{1}, \ldots, x_{m-1}, x_{m}\right)$ e consideremos a família $\mathcal{G}=\left\{A_{x_{1}, \ldots, x_{m-1}}: x_{i} \in E_{i} \mathrm{e}\left\|x_{i}\right\| \leq 1, \forall i \in\{1, \ldots, m-1\}\right\}$. Então, por um raciocínio similar ao feito anteriormente nesta demonstração, para cada $x_{m}$ em $E_{m}$ com $\left\|x_{m}\right\| \leq 1$ existe $M_{x_{m}}$ satisfazendo $\left\|A_{x_{1}, \ldots, x_{m}}\left(x_{m}\right)\right\|=\left\|A\left(x_{1}, \ldots, x_{m}\right)\right\| \leq M_{x_{m}}$, para cada $\left(x_{1}, \ldots, x_{m-1}\right) \in E_{1} \times \cdots \times E_{m-1}$ com $\left\|x_{i}\right\| \leq 1, \forall i \in\{1, \ldots, m-1\}$. Assim, novamente pelo princípio da limitação uniforme (1.6), $\left\|A_{x_{1}, \ldots, x_{m}}\right\| \leq M$, para cada $A_{x_{1}, \ldots, x_{m}}$ em $\mathcal{G}$. Isto é, $\left\|x_{i}\right\| \leq 1, \forall i \in\{1, \ldots, m\}$ implica em $\left\|A\left(x_{1}, \ldots, x_{m}\right)\right\| \leq M$, e portanto $A$ é contínua.

Corolário 2.8. Seja $A \in \mathcal{L}_{a}\left(X_{1}, \ldots, X_{m} ; Y\right)$ onde $X_{i}$ é de dimensão finita, para cada $i \in\{1, \ldots, m\}$. Então A é contínua.

\section{Demonstração:}

Para cada $i \in\{1, \ldots, m\}$ o espaço $X_{i}$ é de Banach por ser de dimensão finita, e pelo mesmo motivo temos também que $A$ é contínua em cada variável. Portanto, pelo Teorema anterior, $A$ é contínua. 
Teorema 2.9. Sejam $X_{1}, \ldots, X_{m}, Y_{1}, \ldots, Y_{n}, Z$ espaços normados. Então os espaços

$$
\mathcal{L}\left(X_{1}, \ldots, X_{m}, Y_{1}, \ldots, Y_{n} ; Z\right) \text { e } \mathcal{L}\left(X_{1}, \ldots, X_{m} ; \mathcal{L}\left(Y_{1}, \ldots, Y_{n} ; Z\right)\right)
$$

são isométricos.

\section{Demonstração:}

Vamos mostrar que a função

$$
\phi: \mathcal{L}\left(X_{1}, \ldots, X_{m}, Y_{1}, \ldots, Y_{n} ; Z\right) \rightarrow \mathcal{L}\left(X_{1}, \ldots, X_{m} ; \mathcal{L}\left(Y_{1}, \ldots, Y_{n} ; Z\right)\right)
$$

definida por $\phi(A)(x)(y) \doteq A(x, y)$, para cada $x=\left(x_{1}, \ldots, x_{m}\right) \in X_{1} \times \cdots \times X_{m}$ e cada $y=\left(y_{1}, \ldots, y_{n}\right) \in Y_{1} \times \cdots \times Y_{n}$, é uma isometria.

Precisamos mostrar que a definição de $\phi$ acima é coerente: seja

$$
A \in \mathcal{L}\left(X_{1}, \ldots, X_{m}, Y_{1}, \ldots, Y_{n} ; Z\right)
$$

e vamos mostrar que de fato

$$
\phi(A) \in \mathcal{L}\left(X_{1}, \ldots, X_{m} ; \mathcal{L}\left(Y_{1}, \ldots, Y_{n} ; Z\right)\right) .
$$

Fixemos $x=\left(x_{1}, \ldots, x_{m}\right) \in X_{1} \times \cdots \times X_{m}$. É evidente que a aplicação $\phi(A)(x)$ é n-linear, e é contínua de norma menor ou igual a $\|A\|\left\|x_{1}\right\| \ldots\left\|x_{m}\right\|$ pois para cada $y=$ $\left(y_{1}, \ldots, y_{n}\right) \in Y_{1} \times \cdots \times Y_{n},\|\phi(A)(x)(y)\|=\|A(x, y)\| \leq\|A\|\left\|x_{1}\right\| \ldots\left\|x_{m}\right\|\left\|y_{1}\right\| \ldots\left\|y_{n}\right\|$. É facil ver que $\phi(A)$ é m-linear. Então pelo fato de que para $x=\left(x_{1}, \ldots, x_{m}\right) \in X_{1} \times \cdots \times X_{m}$ arbitrário temos $\|\phi(A)(x)\| \leq\|A\|\left\|x_{1}\right\| \ldots\left\|x_{m}\right\|$, segue que $\phi(A)$ é contínua. Mostramos portanto que $\phi(A) \in \mathcal{L}\left(X_{1}, \ldots, X_{m} ; \mathcal{L}\left(Y_{1}, \ldots, Y_{n} ; Z\right)\right)$ e que $\|\phi(A)\| \leq\|A\|$, para cada $A \in \mathcal{L}\left(X_{1}, \ldots, X_{m}, Y_{1}, \ldots, Y_{n} ; Z\right)$.

$\phi$ é linear, pois se $A, B \in \mathcal{L}\left(X_{1}, \ldots, X_{m}, Y_{1}, \ldots, Y_{n} ; Z\right)$ e $\lambda \in \mathbb{K}$, então para cada $(x, y) \in X_{1} \times \cdots \times X_{m} \times Y_{1} \times \cdots \times Y_{n}$ temos que

$$
\phi(A+\lambda B)(x)(y)=A(x, y)+\lambda B(x, y)=\phi(A)(x)(y)+\lambda \phi(B)(x)(y) .
$$

Como $\|\phi(A)\| \leq\|A\|$ para cada $A \in \mathcal{L}\left(X_{1}, \ldots, X_{m}, Y_{1}, \ldots, Y_{n} ; Z\right)$, segue que $\phi$ é contínua.

Para verificar que $\phi$ é injetora, basta mostrar que $\phi^{-1}(0)=\{0\}$. De fato, se $\phi(A)=0$, temos que $A(x, y)=\phi(A)(x)(y)=0$, para cada $x \in X_{1} \times \ldots \times X_{m}, y \in Y_{1} \times \ldots \times Y_{n}$, isto é, $A=0$. 
Para provar que $\phi$ é sobrejetora fixemos $B \in \mathcal{L}\left(X_{1}, \ldots, X_{m} ; \mathcal{L}\left(Y_{1}, \ldots, Y_{n} ; Z\right)\right)$ e vamos mostrar que $A: X_{1} \times \cdots \times X_{m} \times Y_{1} \times \cdots \times Y_{n} \rightarrow Z$ definida por $A(x, y) \doteq B(x)(y)$ é tal que $A \in \mathcal{L}\left(X_{1}, \ldots, X_{m}, Y_{1}, \ldots, Y_{n} ; Z\right)$ e $\phi(A)=B$. É fácil ver que $A$ é multilinear. Vamos mostrar que, para cada $(x, y)=\left(x_{1}, \ldots, x_{m}, y_{1}, \ldots, y_{n}\right) \in X_{1} \times \cdots \times X_{m} \times Y_{1} \times \cdots \times Y_{n}$, $A$ é contínua em $(x, y)$. De fato,

$$
\|A(x, y)\|=\|B(x)(y)\| \leq\|B(x)\|\left\|y_{1}\right\| \ldots\left\|y_{n}\right\| \leq\|B\|\left\|x_{1}\right\| \ldots\left\|x_{m}\right\|\left\|y_{1}\right\| \ldots\left\|y_{n}\right\|
$$

observando-se que a primeira desigualdade é devida à continuidade de $B(x)$, e a segunda desigualdade é devida à continuidade de $B$. Podemos concluir então que $A$ é contínua, e evidentemente $\phi(A)=B$, pela definição de $\phi$. Observe também que $\|A\| \leq\|B\|=\|\phi(A)\|$. Como acabamos de mostrar que $\phi$ é uma bijeção entre $\mathcal{L}\left(X_{1}, \ldots, X_{m}, Y_{1}, \ldots, Y_{n} ; Z\right)$ e $\mathcal{L}\left(X_{1}, \ldots, X_{m} ; \mathcal{L}\left(Y_{1}, \ldots, Y_{n} ; Z\right)\right)$, podemos afirmar que

$$
\|A\| \leq\|\phi(A)\|, \forall A \in \mathcal{L}\left(X_{1}, \ldots, X_{m}, Y_{1}, \ldots, Y_{n} ; Z\right) .
$$

Mostramos anteriormente que $\|\phi(A)\| \leq\|A\|, \forall A \in \mathcal{L}\left(X_{1}, \ldots, X_{m}, Y_{1}, \ldots, Y_{n} ; Z\right)$, e portanto $\phi$ preserva norma.

Como $\phi$ é linear, contínua, bijetora, e preserva norma, segue que $\phi^{-1}$ é contínua e $\phi$ é uma isometria, como queríamos.

Uma consequência notável deste Teorema é o seguinte corolário:

Corolário 2.10. Sejam $X_{1}, \ldots, X_{m}$ espaços normados e $F$ um espaço de Banach.

Então $\mathcal{L}\left(X_{1}, \ldots, X_{m} ; F\right)$ é espaço de Banach.

\section{Demonstração:}

Vamos mostrar que $\mathcal{L}\left(X_{1}, \cdots, X_{m} ; F\right)$ é completo por indução.

É sabido, para o caso $m=1$, que $\mathcal{L}\left(X_{1} ; F\right)$ é completo. Vamos supor que $\mathcal{L}\left(X_{1}, \ldots, X_{m} ; F\right)$ é completo, e precisamos mostrar que $\mathcal{L}\left(X_{0}, X_{1}, \ldots, X_{m} ; F\right)$ é completo.

$\mathcal{L}\left(X_{0} ; \mathcal{L}\left(X_{1}, \ldots, X_{m} ; F\right)\right)$ é completo pelo fato de $\mathcal{L}\left(X_{1}, \ldots, X_{m} ; F\right)$ ser espaço de Banach, e pelo Teorema anterior $\mathcal{L}\left(X_{0} ; \mathcal{L}\left(X_{1}, \ldots, X_{m} ; F\right)\right)$ é isométrico a $\left.\mathcal{L}\left(X_{0}, X_{1}, \ldots, X_{m} ; F\right)\right)$. Pela preservação da completude por isometrias, concluímos a demonstração. 


\subsection{Aplicações Multilineares Simétricas}

Vamos estudar agora este importante subespaço das aplicações multilineares. Mais adiante veremos que os espaços de polinômios são isomorfos aos espaços de transformações multilineares simétricas.

Definição 2.11. Sejam $X, Y \mathbb{K}$-espaços vetoriais. Uma aplicação $m$-linear $A: X^{m} \rightarrow Y$ é dita simétrica se para cada $x_{1}, \ldots, x_{m} \in X$ tivermos que

$$
A\left(x_{1}, \ldots, x_{m}\right)=A\left(x_{\sigma(1)}, \ldots, x_{\sigma(m)}\right), \forall \sigma \in S_{m},
$$

onde $S_{m}$ é o grupo de permutações de $m$ elementos.

Denotamos por $\mathcal{L}_{\text {as }}\left({ }^{m} X ; Y\right)$ o espaço das transformações $m$-lineares simétricas de $X^{m}$ em $Y$, e se $X$ e $Y$ forem normados denotamos por $\mathcal{L}_{s}\left({ }^{m} X ; Y\right)$ o espaço das transformações m-lineares simétricas contínuas de $X^{m}$ em $Y$. Quando $Y=\mathbb{K}$, escrevemos $\mathcal{L}_{\text {as }}\left({ }^{m} X ; \mathbb{K}\right) \doteq \mathcal{L}_{\text {as }}\left({ }^{m} X\right)$ e $\mathcal{L}_{s}\left({ }^{m} X ; \mathbb{K}\right) \doteq \mathcal{L}_{s}\left({ }^{m} X\right)$

Para simplificar notações, utilizamos a seguinte convenção, para qualquer $A$ multilinear:

$$
A(\underbrace{x_{1}, \ldots, x_{1}}_{n_{1} \text { vezes }}, \underbrace{x_{2}, \ldots, x_{2}}_{n_{2} \text { vezes }}, \ldots, \underbrace{x_{k}, \ldots, x_{k}}_{n_{k} \text { vezes }}) \doteq A x_{1}^{n_{1}} \ldots x_{k}^{n_{k}}
$$

A seguir está enunciada a fórmula de Leibniz, cuja demonstração omitiremos pois é muito técnica e está bem escrita em [19], mas que tem importantes consequências para o estudo dos polinômios em espaços de Banach:

Teorema 2.12 (Fórmula de Leibniz). Sejam $X, Y \mathbb{K}$-espaços vetoriais e $A \in \mathcal{L}_{a s}\left({ }^{m} X ; Y\right)$, e seja $k \in \mathbb{N}$. Então para cada $x_{1}, \ldots, x_{k} \in X$ vale que

$$
A\left(x_{1}+\cdots+x_{k}\right)^{m}=\sum_{n_{1}+\cdots+n_{k}=m} \frac{m !}{n_{1} ! \ldots n_{k} !} A x_{1}^{n_{1}} \ldots x_{k}^{n_{k}}
$$

onde cada $n_{1}, \ldots, n_{k} \in \mathbb{N}_{0}$.

Usaremos com mais frequência a versão da fórmula de Leibniz para o caso $k=2$ :

Corolário 2.13 (Fórmula binomial). Sejam $X, Y \mathbb{K}$-espaços vetoriais e $A \in \mathcal{L}_{a s}\left({ }^{m} X ; Y\right)$. Então para cada $x, y \in X$ vale que

$$
A(x+y)^{m}=\sum_{k=0}^{m}\left(\begin{array}{c}
m \\
k
\end{array}\right) A x^{m-k} y^{k} .
$$




\section{Demonstração:}

Basta observar que $\sum_{n_{1}+n_{2}=m} \frac{m !}{n_{1} ! n_{2} !} A x^{n_{1}} y^{n_{2}}=\sum_{n=0}^{m} \frac{m !}{n !(m-n) !} A x^{n} y^{m-n}$ e o resultado é a aplicação direta da fórmula de Leibniz.

Outra consequência da fórmula de Leibniz é a fórmula de polarização, apresentada no próximo Teorema.

Teorema 2.14 (Fórmula de polarização). Sejam $X, Y \mathbb{K}$-espaços vetoriais e $A \in \mathcal{L}_{a s}\left({ }^{m} X ; Y\right)$.

Então para cada $x_{1}, \ldots, x_{m} \in X$ vale que

$$
A\left(x_{1}, \ldots, x_{m}\right)=\frac{1}{m ! 2^{m}} \sum_{\substack{\epsilon_{i}= \pm 1 \\ 1 \leq i \leq m}} \epsilon_{1} \ldots \epsilon_{m} A\left(\epsilon_{1} x_{1}+\cdots+\epsilon_{m} x_{m}\right)^{m} .
$$

\section{Demonstração:}

Pela fórmula de Leibiniz, temos para cada $x_{1}, \ldots, x_{m} \in X$ que

$$
\begin{aligned}
A\left(\epsilon_{1} x_{1}+\cdots+\epsilon_{m} x_{m}\right)^{m} & =\sum_{n_{1}+\cdots+n_{m}=m} \frac{m !}{n_{1} ! \ldots n_{m} !} A\left(\epsilon_{1} x_{1}\right)^{n_{1}} \ldots\left(\epsilon_{m} x_{m}\right)^{n_{m}} \\
& =\sum_{n_{1}+\cdots+n_{m}=m} \frac{m !}{n_{1} ! \cdots n_{m} !} \epsilon_{1}^{n_{1}} \ldots \epsilon_{m}^{n_{m}} A x_{1}^{n_{1}} \ldots x_{m}^{n_{m}} .
\end{aligned}
$$

Então

$$
\begin{aligned}
& \sum_{\substack{\epsilon_{i}= \pm 1 \\
1 \leq i \leq m \\
1 \leq}} \epsilon_{1} \cdots \epsilon_{m} A\left(\epsilon_{1} x_{1}+\cdots+\epsilon_{m} x_{m}\right)^{m} \\
= & \sum_{\substack{\epsilon_{i}= \pm 1 \\
1 \leq i \leq m}} \epsilon_{1} \ldots \epsilon_{m} \sum_{n_{1}+\cdots+n_{m}=m} \frac{m !}{n_{1} ! \ldots n_{m} !} \epsilon_{1}^{n_{1}} \ldots \epsilon_{m}^{n_{m}} A x_{1}^{n_{1}} \ldots x_{m}^{n_{m}} \\
= & \sum_{n_{1}+\cdots+n_{m}=m} \frac{m !}{n_{1} ! \ldots n_{m} !} A x_{1}^{n_{1}} \ldots x_{m}^{n_{m}} \sum_{\substack{\epsilon_{i}= \pm 1 \\
1 \leq i \leq m}} \epsilon_{1}^{n_{1}+1} \ldots \epsilon_{m}^{n_{m}+1} .
\end{aligned}
$$

Quando $n_{j}$ é zero para algum $j \in \mathbb{N}$, temos que $\epsilon_{j}^{n_{j}+1}=\epsilon_{j}^{1}$ e assim

$$
\sum_{\substack{\epsilon_{i}= \pm 1 \\ 1 \leq i \leq m}} \epsilon_{1}^{n_{1}+1} \ldots \epsilon_{m}^{n_{m}+1}=\sum_{\epsilon_{j}= \pm 1} \epsilon_{j} \sum_{\substack{\epsilon_{i}= \pm 1 \\ i \neq j}} \epsilon_{1}^{n_{1}+1} \ldots \epsilon_{j-1}^{n_{j-1}+1} \epsilon_{j+1}^{n_{j+1}+1} \ldots \epsilon_{m}^{n_{m}+1}=0 .
$$


Desta forma, todas as parcelas da somatória

$$
\sum_{n_{1}+\cdots+n_{m}=m} \frac{m !}{n_{1} ! \ldots n_{m} !} A x_{1}^{n_{1}} \ldots x_{m}^{n_{m}} \sum_{\substack{\epsilon_{i}= \pm 1 \\ 1 \leq i \leq m}} \epsilon_{1}^{n_{1}+1} \ldots \epsilon_{m}^{n_{m}+1}
$$

se anulam, exceto a parcela em que $n_{1}=\cdots=n_{m}=1$. Como

$$
\sum_{\substack{\epsilon_{i}= \pm 1 \\ 1 \leq i \leq m}} \epsilon_{1}^{1+1} \ldots \epsilon_{m}^{1+1}=\sum_{\substack{\epsilon_{i}= \pm 1 \\ 1 \leq i \leq m}} \epsilon_{1}^{2} \ldots \epsilon_{m}^{2}=\sum_{\substack{\epsilon_{i}= \pm 1 \\ 1 \leq i \leq m}} 1=2^{m}
$$

podemos concluir que

$$
\begin{aligned}
& \sum_{\substack{\epsilon_{i}= \pm 1 \\
1 \leq i \leq m}} \epsilon_{1} \cdots \epsilon_{m} A\left(\epsilon_{1} x_{1}+\cdots+\epsilon_{m} x_{m}\right)^{m} \\
= & \sum_{n_{1}+\cdots+n_{m}=m} \frac{m !}{n_{1} ! \ldots n_{m} !} A x_{1}^{n_{1}} \ldots x_{m}^{n_{m}} \sum_{\substack{\epsilon_{i}= \pm 1 \\
1 \leq i \leq m}} \epsilon_{1}^{n_{1}+1} \ldots \epsilon_{m}^{n_{m}+1} \\
= & m ! A\left(x_{1}, \ldots, x_{m}\right) 2^{m},
\end{aligned}
$$

de onde podemos deduzir a fórmula de polarização.

Observação: Através dessa fórmula podemos concluir que uma transformação multilinear simétrica em $X$ é unicamente determinada por seus valores na diagonal de $X^{m}$, que é definida como o conjunto $\left\{\left(x_{1}, \cdots, x_{m}\right) \in X^{m}: x_{1}=\cdots=x_{m}\right\}$.

\subsection{Polinômios}

Nesta seção vamos, a partir das transformações multilineares, definir os polinômios homogêneos e estabelecer a relação biunívoca entre os polinômios homogêneos e as transformações multilineares simétricas.

Definição 2.15. Sejam $X$ e $Y \mathbb{K}$-espaços vetoriais. Dizemos que $P: X \rightarrow Y$ é um polinômio homogêneo de grau $\boldsymbol{m}$ (ou polinômio $\boldsymbol{m}$-homogêneo) e escrevemos $P \in \mathcal{P}_{a}\left({ }^{m} X ; Y\right)$ se existir $A \in \mathcal{L}_{a}\left({ }^{m} X ; Y\right)$ tal que $P(x)=A x^{m}$, para cada $x \in X$. 
Dizemos então que A é uma transformação m-linear associada a $P$, e que $P$ é $o$ polinômio m-homogêneo associado a $A$, e denotamos $P=\hat{A}$.

Quando $Y=\mathbb{K}$, denotamos $\mathcal{P}_{a}\left({ }^{m} X\right) \doteq \mathcal{P}_{a}\left({ }^{m} X ; \mathbb{K}\right)$.

É fácil verificar que o conjunto $\mathcal{P}_{a}\left({ }^{m} X ; Y\right)$ é um $\mathbb{K}$-espaço vetorial, bem como $\mathcal{P}_{a}(X ; Y)$, definido a seguir:

Definição 2.16. Sejam $X$ e $Y \mathbb{K}$-espaços vetoriais. Dizemos que $P: X \rightarrow Y$ é um polinômio (de grau $m$ ) se existem $m \in \mathbb{N}$ e $P_{i} \in \mathcal{P}_{a}\left({ }^{i} X ; Y\right), i \in\{0, \ldots, m\}, P_{m} \neq 0$ tais que

$$
P=P_{0}+P_{1}+\cdots+P_{m}
$$

Denotamos por $\mathcal{P}_{a}(X ; Y)$ o $\mathbb{K}$-espaço vetorial dos polinômios (de grau qualquer), e quando $Y=\mathbb{K}$ escrevemos $\mathcal{P}_{a}(X) \doteq \mathcal{P}_{a}(X ; \mathbb{K})$.

Na próxima proposição, vamos verificar que são únicos os polinômios que satisfazem 2.1. Observemos que, se $X$ e $Y$ são $\mathbb{K}$-espaços vetoriais e $P \in \mathcal{P}\left({ }^{m} X ; Y\right)$, então para cada $x \in X$ e cada $\lambda \in \mathbb{K}$ temos que $P(\lambda x)=\lambda^{m} P(x)$.

Proposição 2.17. Sejam $X$ e $Y \mathbb{K}$-espaços vetoriais. Então $\mathcal{P}_{a}(X ; Y)$ é a soma direta algébrica dos espaços $\mathcal{P}_{a}\left({ }^{m} X ; Y\right)$, com $m \in \mathbb{N}$.

\section{Demonstração:}

Basta provar que, se $P \in \mathcal{P}(X ; Y)$ é tal que $P=P_{0}+\cdots+P_{m}=0, P_{i} \in \mathcal{P}_{a}\left({ }^{i} X ; Y\right)$ para cada $i \in\{0, \ldots, m\}$, então $P_{0}=\cdots=P_{m}=0$.

Para cada $x \in X$ e $r>0$ temos que

$$
\begin{aligned}
P_{0}(r x)+P_{1}(r x)+\cdots+P_{m}(r x)=0 & \Rightarrow r^{0} P_{0}(x)+r P_{1}(r x)+\cdots+r^{m} P_{m}(x)=0 \\
& \Rightarrow \frac{1}{r^{m}} P_{0}(x)+\frac{1}{r^{m-1}} P_{1}(x)+\cdots+P_{m}(x)=0 .
\end{aligned}
$$

Como $r>0$ é arbitrário e o lado esquerdo desta última igualdade converge a $P_{m}(x)$ quando $r \rightarrow \infty$, temos que $P_{m}(x)=0$, para cada $x \in X$. Assim, $P=P_{0}+\cdots+P_{m-1}=0$. Repetindo este recurso, podemos concluir que $P_{0}=\cdots=P_{m}=0$.

Proposição 2.18. Seja $P \in \mathcal{P}_{a}\left({ }^{m} X, Y\right)$, então existe uma única $A \in \mathcal{L}_{a s}\left({ }^{m} E ; F\right)$ tal que para cada $x \in X, P(x)=A x^{m}$. 
Demonstração: Seja $P \in \mathcal{P}_{a}\left({ }^{m} E ; F\right)$. Então existe $A \in \mathcal{L}_{a}\left({ }^{m} E ; F\right) \operatorname{com} P(x)=$ $A x^{m}$. Vamos gerar uma aplicação simétrica a partir desta $A$. Defino, para cada $x_{1}, \ldots, x_{m} \in$ $X$

$$
A_{s}\left(x_{1}, \ldots, x_{m}\right) \doteq \frac{1}{m !} \sum_{\sigma \in S_{m}} A\left(x_{\sigma(1)}, \ldots, x_{\sigma(m)}\right) .
$$

É fácil verificar que $A_{s} \in \mathcal{L}_{a s}\left({ }^{m} X ; Y\right)$ e que $A_{s} x^{m}=A x^{m}=P(x)$ para cada $x \in X$.

Vamos verificar a unicidade desta solução. Se $A_{s}^{\prime} \in \mathcal{L}_{a s}\left({ }^{m} E ; F\right)$ é tal que $P(x)=A_{s}^{\prime} x^{m}$ para cada $x \in X$, então $A_{s}^{\prime} x^{m}=A_{s} x^{m}$, para cada $x \in X$, e pela fórmula de polarização (2.14) concluimos que $A_{s}^{\prime}=A_{s}$.

Observação: A aplicação $A_{s}$ definida na demontração acima é dita a simetrização de $A$.

Considerando este resultado, podemos definir:

Definição 2.19. Sejam $X$ e $Y \mathbb{K}$-espaços vetoriais, e seja $P \in \mathcal{P}_{a}\left({ }^{m} X ; Y\right)$. Denotamos por $\check{P}$ a única transformação m-linear simétrica de $X$ em $Y$ tal que, para cada $x \in X$, $\check{P} x^{m}=P(x)$. Dizemos que $\check{P}$ é a tranformação m-linear simétrica associada a $P$.

Observemos que, dado um polinômio homogêneo $P$, a fórmula de polarização $(2.14)$ nos permite obter $\check{P}$.

Proposição 2.20. Sejam $X$ e $Y$ espaços vetoriais. Então a função $\left.\varphi: \mathcal{L}_{a s}{ }^{m} X ; Y\right) \rightarrow$ $\mathcal{P}_{a}\left({ }^{m} X ; Y\right)$ definida por $\varphi(A) \doteq \hat{A}$ é um isomorfismo sobrejetor de espaços vetoriais.

\section{Demonstração:}

A aplicação $\varphi$ é bijetora pela proposição 2.18. Assim, basta verificar $\varphi$ é linear. Sejam $A_{1}, A_{2} \in L_{a s}\left({ }^{m} E ; F\right)$ e $\lambda \in \mathbb{K}$. Então para cada $x \in X$,

$$
\begin{gathered}
\varphi\left(A_{1}+\lambda A_{2}\right)(x)=\left(\widehat{A_{1}+\lambda A_{2}}\right)(x)=\left(A_{1}+\lambda A_{2}\right) x^{m}=A_{1} x^{m}+\left(\lambda A_{2}\right) x^{m} \\
=A_{1} x^{m}+\lambda\left(A_{2} x^{m}\right)=\widehat{A_{1}}(x)+\lambda \widehat{A_{2}}(x)=\varphi\left(A_{1}\right)(x)+\lambda \varphi\left(A_{2}\right)(x),
\end{gathered}
$$

como queríamos.

Quando $X$ e $Y$ são espaços normados, denotamos por $\mathcal{P}\left({ }^{m} X ; Y\right)$ o subespaço vetorial de $\mathcal{P}_{a}\left({ }^{m} X ; Y\right)$ formado pelos polinômios $m$-homogêneos contínuos, e por $\mathcal{P}(X ; Y)$ o 
subespaço vetorial de $\mathcal{P}_{a}(X ; Y)$ formado pelos polinômios contínuos. Temos a seguinte caracterização para os polinômios homogêneos contínuos, semelhante aos casos linear e multilinear:

Teorema 2.21. Sejam $X, Y$ espaços normados, $A \in \mathcal{L}_{a s}\left({ }^{m} X ; Y\right)$ e $P \in \mathcal{P}_{a}\left({ }^{m} X ; Y\right)$ com $\hat{A}=P$. Então são equivalentes as seguintes afirmações:

1) $A \in \mathcal{L}_{s}\left({ }^{m} X ; Y\right)$;

2) $P \in \mathcal{P}\left({ }^{m} X ; Y\right)$;

3) Pé contínuo na origem;

4) Existe $M>0$ tal que $\|P(x)\| \leq M\|x\|^{m}$, para cada $x \in X$.

\section{Demontração:}

$((1) \Rightarrow(2))$ Definindo $f: x \in X \rightarrow(x, \ldots, x) \in X^{m}$, temos que $P=A \circ f$. Como $f$ e $A$ são contínuas, $P$ também é.

$((2) \Rightarrow(3))$ Trivial.

$((3) \Rightarrow(4)) P(0)=0$, e por hipótese $P$ é contínuo na origem. Então dado $\epsilon=1$ existe $\delta>0$ tal que $\|x\|<\delta \Rightarrow\|P(x)\| \leq 1$. Para cada $x \in X$ não nulo, temos que $\left\|\frac{\delta x}{2\|x\|}\right\|<\delta$ e desta forma $\left\|P\left(\frac{\delta}{2} \frac{x}{\|x\|}\right)\right\| \leq 1$. Como

$$
\left\|P\left(\frac{\delta}{2} \frac{x}{\|x\|}\right)\right\|=\frac{\delta^{m}}{2^{m}}\left\|P\left(\frac{x}{\|x\|}\right)\right\|=\frac{\delta^{m}}{2^{m}\|x\|^{m}}\|P(x)\|,
$$

segue que $\|P(x)\| \leq \frac{2^{m}}{\delta^{m}}\|x\|^{m}$, como queríamos.

$$
((4) \Rightarrow(1)) \text { Seja } M>0 \text { tal que }\|P(x)\| \leq M\|x\|^{m} \text {, para cada } x \in X \text {. Sejam }
$$


$x_{1}, \ldots, x_{m} \in X$ satisfazendo $\left\|x_{i}\right\| \leq \frac{1}{m}$, para cada $i \in\{1, \ldots, m\}$. Então

$$
\begin{aligned}
\left\|A\left(x_{1}, \ldots, x_{m}\right)\right\| & \leq \frac{1}{m ! 2^{m}} \sum_{\substack{\epsilon_{i}= \pm 1 \\
1 \leq i \leq m}}\left|\epsilon_{1} \ldots \epsilon_{m}\right|\left\|A\left(\epsilon_{1} x_{1}+\cdots+\epsilon_{m} x_{m}\right)^{m}\right\| \\
& \leq \frac{1}{m ! 2^{m}} \sum_{\epsilon_{i}= \pm 1}\left\|P\left(\epsilon_{1} x_{1}+\cdots+\epsilon_{m} x_{m}\right)\right\| \\
& \leq \frac{1}{m ! 2^{m}} \sum_{\epsilon_{i}= \pm 1} M\left\|\left(\epsilon_{1} x_{1}+\cdots+\epsilon_{m} x_{m}\right)\right\|^{m} \\
& \leq \frac{1}{m ! 2^{m}} \sum_{\epsilon_{i}= \pm 1}^{1 \leq i \leq m} \\
& =\frac{1}{m ! 2^{m}} 2^{m} M=\frac{M}{m !} .
\end{aligned}
$$

Sejam agora $x_{1}, \ldots, x_{m} \in X$ não nulos. Então para cada $i \in\{1, \ldots, m\},\left\|\frac{x_{i}}{m\left\|x_{i}\right\|}\right\| \leq \frac{1}{m}$. Pelo que acabamos de mostrar temos que $\left\|A\left(\frac{x_{1}}{m\left\|x_{1}\right\|}, \ldots, \frac{x_{m}}{m\left\|x_{m}\right\|}\right)\right\| \leq \frac{M}{m !}$. Então

$$
\frac{\left\|A\left(x_{1}, \ldots, x_{m}\right)\right\|}{m^{m}\left\|x_{1}\right\| \ldots\left\|x_{m}\right\|}=\left\|A\left(\frac{x_{1}}{m\left\|x_{1}\right\|}, \ldots, \frac{x_{m}}{m\left\|x_{m}\right\|}\right)\right\| \leq \frac{M}{m !}
$$

e portanto

$$
\left\|A\left(x_{1}, \ldots, x_{m}\right)\right\| \leq \frac{M m^{m}}{m !}\left\|x_{1}\right\| \ldots\left\|x_{m}\right\| .
$$

Como a desigualdade acima vale também para o caso em que $x_{i}=0$ para algum $i \in\{1, \ldots, m\}$, podemos concluir pelo Teorema 2.2 que $A$ é contínua.

Como consequência do corolário 2.3, temos o seguinte resultado análogo ao caso multilinear:

Proposição 2.22. Sejam $X$ e $Y$ espaços normados, $U \subset X$ um conjunto limitado $e$ $P \in \mathcal{P}\left({ }^{m} X ; Y\right)$. Então existe uma constante $M \geq 0$ tal que para cada $x, y \in U$,

$$
\|P(x)-P(y)\| \leq M\|x-y\|
$$

e consequentemente $P$ é uniformemente contínuo sobre conjuntos limitados. 


\section{Demonstração:}

Seja $A \in \mathcal{L}\left({ }^{m} X ; Y\right)$ tal que $\hat{A}=P$. Pelo corolário 2.3 , temos que existe $M \geq 0$ tal que para cada $x_{1}, \ldots, x_{m}, y_{1}, \ldots, y_{m} \in U$,

$$
\left\|A\left(x_{1}, \ldots, x_{m}\right)-A\left(y_{1}, \ldots, y_{m}\right)\right\| \leq M\left\|\left(x_{1}-y_{1}, \ldots, x_{m}-y_{m}\right)\right\| .
$$

Em particular, para cada $x, y \in U$

$$
\|P(x)-P(y)\|=\|A(x, \ldots, x)-A(y, \ldots, y)\| \leq M\|(x-y, \ldots, x-y)\|=M\|x-y\|,
$$

como queríamos.

Sobre o $\mathbb{K}$-espaço vetorial $\left.\mathcal{P}{ }^{m} X ; Y\right)$ podemos definir a norma dada pela proposição seguinte.

Proposição 2.23. Sejam $X, Y$ espaços normados. Então $\|\cdot\|: \mathcal{P}\left({ }^{m} X ; Y\right) \rightarrow \mathbb{R}$ definida por

$$
\|P\| \doteq \sup \{\|P(x)\|:\|x\| \leq 1\}
$$

é uma norma sobre $\mathcal{P}\left({ }^{m} X ; Y\right)$, e para cada $P \in \mathcal{P}\left({ }^{m} X ; Y\right)$ temos que

$$
\|P\|=\inf \left\{M \geq 0:\|P(x)\| \leq M\|x\|^{m}, \forall x \in X\right\} .
$$

\section{Demonstração:}

A demonstração é análoga à da proposição 2.5.

Estabelecida a norma no espaço dos polinômios homogêneos contínuos, temos o seguinte aprimoramento da proposição 2.20 :

Proposição 2.24. Sejam $X$ e $Y$ espaços normados. Então para cada $A \in \mathcal{L}_{s}\left({ }^{m} X ; Y\right)$ temos que

$$
\|\widehat{A}\| \leq\|A\| \leq \frac{m^{m}}{m !}\|\widehat{A}\|
$$

e a função $\phi: A \in \mathcal{L}_{s}\left({ }^{m} X ; Y\right) \rightarrow \hat{A} \in \mathcal{P}\left({ }^{m} X ; Y\right)$ é um isomorfismo topológico.

Demonstração: Vimos na proposição 2.20 que $\varphi: A \in \mathcal{L}_{a s}\left({ }^{m} X ; Y\right) \rightarrow \hat{A} \in \mathcal{P}_{a}\left({ }^{m} X ; Y\right)$ é um isomorfismo de espaços vetoriais. Como $\phi=\left.\varphi\right|_{\left.\mathcal{L}_{s}{ }^{m} X ; Y\right)}$, $\phi$ é linear e injetora. Se $P$ pertence a $\mathcal{P}\left({ }^{m} X ; Y\right)$, pela proposição 2.18 existe $A \in \mathcal{L}_{a s}\left({ }^{m} X ; Y\right)$ tal que $\widehat{A}=P$. Pela proposição $2.21 A$ é contínua, e podemos concluir que $\phi$ é sobrejetora. 
Fixemos $A \in \mathcal{L}_{s}\left({ }^{m} X ; Y\right)$ e vamos verificar a desigualdade em questão. Para $m=0$ ou 1 , a desigualdade vale trivialmente. Consideremos então $m \geq 2$. Temos para cada $x \in X$ que $\|\widehat{A}(x)\|=\left\|A x^{m}\right\| \leq\|A\|\|x\|^{m}$. Logo, $\|\widehat{A}\| \leq\|A\|$. Sejam $x_{1}, \ldots, x_{m} \in E$, com $\left\|x_{i}\right\| \leq 1$ para cada $i \in\{1, \ldots, m\}$. Então

$$
\begin{aligned}
\left\|A\left(x_{1}, \ldots, x_{m}\right)\right\| & \leq \frac{1}{m ! 2^{m}} \sum_{\substack{\epsilon_{i}= \pm 1 \\
1 \leq i \leq m}}\left|\epsilon_{1} \ldots \epsilon_{m}\right|\left\|A\left(\epsilon_{1} x_{1}+\cdots+\epsilon_{m} x_{m}\right)^{m}\right\| \\
& \leq \frac{1}{m ! 2^{m}} \sum_{\substack{\epsilon_{i}= \pm 1 \\
1 \leq i \leq m}}\left\|\widehat{A}\left(\epsilon_{1} x_{1}+\cdots+\epsilon_{m} x_{m}\right)\right\| \\
& \leq \frac{1}{m ! 2^{m}} \sum_{\epsilon_{i}= \pm 1}\|\widehat{A}\|\left\|\epsilon_{1} x_{1}+\cdots+\epsilon_{m} x_{m}\right\|^{m} \\
& \leq \frac{1}{m ! 2^{m}} \sum_{\epsilon_{i}= \pm 1}\|\widehat{A}\|\left(\left\|\epsilon_{1} x_{1}\right\|+\cdots+\left\|\epsilon_{m} x_{m}\right\|\right)^{m} \\
& \leq \frac{1}{m ! 2^{m}} 2^{m}\|\widehat{A}\| m^{m}=\frac{m^{m}}{m !}\|A\|
\end{aligned}
$$

e podemos concluir que $\|A\| \leq \frac{m^{m}}{m !}\|\widehat{A}\|$.

O próximo exemplo ilustra a necessidade da constante $\frac{m^{m}}{m !}$ na inequação 2.2 .

Exemplo 2.25. Seja $A \in \mathcal{L}\left({ }^{m} l_{1} ; \mathbb{K}\right)$ definida para cada $x^{j}=\left(x_{n}^{j}\right)_{n} \in l_{1}, j \in\{1, \ldots, m\}$ por

$$
A\left(x^{1}, \ldots, x^{m}\right)=\frac{1}{m !} \sum_{\sigma \in S_{m}} x_{1}^{\sigma(1)} \ldots x_{m}^{\sigma(m)}
$$

Então $\|A\|=\frac{1}{m !}$ e $\|\hat{A}\|=\frac{1}{m^{m}}$, de forma que $\|A\|=\frac{m^{m}}{m !}\|\hat{A}\|$.

Sem grande dificuldade pode ser verificado esse resultado. Os cálculos detalhados podem ser encontrados em [19].

Corolário 2.26. Sejam $X$ um espaço normado e $F$ um espaço de Banach. Então $\mathcal{P}\left({ }^{m} E ; F\right)$ é um espaço de Banach. Em particular, $\mathcal{P}\left({ }^{m} X\right)$ é um espaço de Banach.

Demonstração: 
Vimos que $\mathcal{L}\left({ }^{m} X ; F\right)$ é completo. Vamos verificar que $\mathcal{L}_{s}\left({ }^{m} X ; F\right) \subset \mathcal{L}\left({ }^{m} X ; F\right)$ é fechado: seja $A \in \overline{\mathcal{L}_{s}\left({ }^{m} X ; F\right)}$, e mostremos que $A$ é simétrica.

Seja então $\left(A_{n}\right)_{n}$ uma sequência em $\mathcal{L}_{s}\left({ }^{m} X ; F\right)$ tal que $\left\|A_{n}-A\right\| \rightarrow 0$. Fixemos $\left(x_{1}, \ldots, x_{m}\right)$ em $X^{m}$. Para cada $n \in \mathbb{N}$, temos

$$
\left\|A_{n}\left(x_{1}, \ldots x_{m}\right)-A\left(x_{1}, \ldots, x_{m}\right)\right\| \leq\left\|A_{n}-A\right\|\left\|x_{1}\right\| \ldots\left\|x_{m}\right\| .
$$

Como $\left\|A_{n}-A\right\| \rightarrow 0$, temos que $A_{n}\left(x_{1}, \ldots x_{m}\right) \rightarrow A\left(x_{1}, \ldots x_{m}\right)$. Mas, para qualquer $\sigma \in S_{m}$

$$
A_{n}\left(x_{1}, \ldots x_{m}\right)=A_{n}\left(x_{\sigma(1)}, \ldots, x_{\sigma(m)}\right) \rightarrow A\left(x_{\sigma(1)}, \ldots, x_{\sigma(m)}\right) .
$$

Então, pela unicidade do limite, $A\left(x_{1}, \cdots, x_{m}\right)=A\left(x_{\sigma(1)}, \cdots, x_{\sigma(m)}\right)$. Como $\left(x_{1}, \ldots, x_{m}\right)$ e $\sigma$ são arbitrários, concluimos que $A$ é simétrica.

Como pela proposição $2.24 \mathcal{P}\left({ }^{m} X ; Y\right)$ é topologicamente isomorfo a $\mathcal{L}_{s}\left({ }^{m} X ; Y\right)$, e portanto é fechado, como queríamos.

Teorema 2.27 (Teorema de Banach-Steinhaus para polinômios homogêneos). Sejam E um espaço de Banach, $Y$ um espaço normado e $\left(P_{n}\right)_{n}$ uma sequência em $\mathcal{P}\left({ }^{m} E ; Y\right)$ tal que, para cada $x \in X$, a sequência $\left(P_{n}(x)\right)_{n}$ é convergente.

Então, chamando $P(x) \doteq \lim _{n \rightarrow \infty} P_{n}(x)$, temos que $P \in \mathcal{P}\left({ }^{m} E ; Y\right)$.

\section{Demonstração:}

Pela fórmula de polarização $(2.14)$, para cada $x_{1}, \ldots, x_{m} \in E$ temos que

$$
\begin{aligned}
\lim _{n \rightarrow \infty} \check{P}_{n}\left(x_{1}, \ldots, x_{m}\right) & =\lim _{n \rightarrow \infty} \frac{1}{m ! 2^{m}} \sum_{\substack{\epsilon_{i}= \pm 1 \\
1 \leq i \leq m}} \epsilon_{1} \ldots \epsilon_{m} \check{P}_{n}\left(\epsilon_{1} x_{1}+\cdots+\epsilon_{m} x_{m}\right)^{m} \\
& =\frac{1}{m ! 2^{m}} \sum_{\substack{\epsilon_{i}= \pm 1 \\
1 \leq i \leq m}} \epsilon_{1} \ldots \epsilon_{m} \lim _{n \rightarrow \infty} P_{n}\left(\epsilon_{1} x_{1}+\cdots+\epsilon_{m} x_{m}\right) \\
& =\frac{1}{m ! 2^{m}} \sum_{\substack{\epsilon_{i}= \pm 1 \\
1 \leq i \leq m}} \epsilon_{1} \ldots \epsilon_{m} P\left(\epsilon_{1} x_{1}+\cdots+\epsilon_{m} x_{m}\right)
\end{aligned}
$$

de forma que $\left(\check{P}_{n}\left(x_{1}, \cdots, x_{m}\right)\right)_{n}$ é convergente.

Definamos, $A\left(x_{1}, \ldots, x_{m}\right) \doteq \lim _{n \rightarrow \infty} \check{P}_{n}\left(x_{1}, \ldots, x_{m}\right)$, para cada $x_{1}, \ldots, x_{m} \in E$. Não é difícil ver que $A \in \mathcal{L}_{a s}\left({ }^{m} E ; Y\right)$, e que para cada $x \in X$ temos que $P(x)=\lim _{n \rightarrow \infty} P_{n}(x)=$ $\lim _{n \rightarrow \infty} \check{P}_{n} x^{m}=A x^{m}$. 
Basta verificar então que $A$ é contínua, o que neste caso equivale pelo Teorema 2.7 a verificar que $A$ é contínua em cada variável; para tal, fixemos $i \in\{1, \ldots, m\}$ e $x_{1}, \ldots, x_{i-1}, x_{i+1}, \ldots, x_{m} \in E$, e quero provar que

$$
T: y \in E \mapsto A\left(x_{1}, \ldots, x_{i-1}, y, x_{i+1}, \ldots, x_{m}\right) \in Y
$$

é contínua. Mas para cada $y \in E$,

$$
T(y)=\lim _{n \rightarrow \infty} \check{P}_{n}\left(x_{1}, \ldots, x_{i-1}, y, x_{i+1}, \ldots, x_{m}\right),
$$

e como a aplicação $\check{P}_{n}\left(x_{1}, \ldots, x_{i-1}, \cdot, x_{i+1}, \ldots, x_{m}\right)$ é contínua podemos concluir pelo Teorema de Banach-Steinhaus para aplicações lineares (1.7) que $T$ é contínua.

Mostramos anteriormente que todo polinômio se escreve de maneira única como soma de polinômios homogêneos. Vamos mostrar que, analogamente, todo polinômio contínuo se escreve de maneira única como soma de polinômios homogêneos contínuos:

Proposição 2.28. Sejam $X$ e $Y$ espaços normados. Então $\mathcal{P}(X ; Y)$ é a soma direta algébrica dos espaços $\mathcal{P}\left({ }^{i} X ; Y\right)$, com $i \in \mathbb{N}$.

\section{Demonstração:}

Seja $P \in \mathcal{P}(X ; Y)$. Pela proposição $2.17, P$ se escreve de maneira única como $P=$ $P_{0}+\cdots+P_{m}$, com cada $P_{i} \in \mathcal{P}_{a}\left({ }^{i} X ; Y\right)$ e $P_{m} \neq 0$. Vamos mostrar que cada $P_{j}$ é contínuo.

O resultado é trivial para $m=0$. Suponhamos o resultado válido para cada polinômio de grau menor que $m$, e vamos mostrar que é válido também para $P$ de grau $m$.

Para cada $x \in X$ e cada $\lambda \in \mathbb{K}$, temos que

$$
P(\lambda x)=\sum_{j=0}^{m} P_{j}(\lambda x)=\sum_{j=0}^{m} \lambda^{j} P_{j}(x)
$$

de forma que se definirmos $Q: X \rightarrow Y$ por

$$
Q(x)=P(\lambda x)-\lambda^{m} P(x)=\sum_{j=0}^{m} \lambda^{j} P_{j}(x)-\lambda^{m} \sum_{j=0}^{m} P_{j}(x)=\sum_{j=0}^{m}\left(\lambda^{j}-\lambda^{m}\right) P_{j}(x)
$$

temos que $Q$ é contínuo de grau menor que $m$. Segue por hipótese de indução que para cada $j \in\{0 \ldots, m-1\}$ o polinômio $\left(\lambda^{j}-\lambda^{m}\right) P_{j}$ é contínuo. Assim, tomando $\lambda \in \mathbb{K}$ tal que $\left(\lambda^{j}-\lambda^{m}\right) \neq 0$, podemos concliur que $P_{j}$ é contínuo para cada $j \in\{0, \ldots, m-1\}$. 
Como $P$ também é contínuo e $P_{m}=P-P_{0}-\ldots-P_{m-1}$, segue que $P_{m}$ é contínuo, e assim concluimos a demonstração.

Proposição 2.29. Sejam $X$ e $Y$ espaços normados, $U \subset X$ um conjunto limitado $e$ $P \in \mathcal{P}(X ; Y)$. Então existe uma constante $M \geq 0$ tal que para cada $x, y \in U$,

$$
\|P(x)-P(y)\| \leq M\|x-y\|
$$

e consequentemente $P$ é uniformemente contínuo sobre conjuntos limitados.

\section{Demonstração:}

Sejam $P_{0} \in \mathcal{P}\left({ }^{0} X ; Y\right), \ldots, P_{m} \in \mathcal{P}\left({ }^{m} X ; Y\right)$ tais que $P=P_{0}+\cdots+P_{m}$. Pela proposição 2.22 temos que existe $M^{\prime} \geq 0$ tal que, para cada $i \in\{0, \ldots, m\}$,

$$
\left\|P_{i}(x)-P_{i}(y)\right\| \leq M^{\prime}\|x-y\|, \forall x, y \in U \text {. }
$$

Para cada $x, y \in U$,

$$
\begin{aligned}
\|P(x)-P(y)\| & =\left\|P_{0}(x)-P_{0}(y)+\cdots+P_{m}(x)-P_{m}(y)\right\| \\
& \leq\left\|P_{0}(x)-P_{0}(y)\right\|+\cdots+\left\|P_{m}(x)-P_{m}(y)\right\| \\
& \leq(m+1) M^{\prime}\|x-y\| .
\end{aligned}
$$

Basta tomar $M \doteq(m+1) M^{\prime}$.

Finalizando esta seção, apresentamos um resultado de [1] sobre polinômios de $\mathcal{P}\left({ }^{N} E\right)$. A demonstração não faz parte deste estudo; esse resultado será utilizado em um exemplo no capítulo 3.

Proposição 2.30. Seja $P \in \mathcal{P}\left({ }^{N} E\right)$, onde $N \geq 1$ e E é um espaço de Banach de dimensão infinita sobre $\mathbb{C}$. Então $P^{-1}(0)$ é um conjunto ilimitado.

\subsection{Polinômios fracamente sequencialmente contínuos}

Nesta seção iniciamos nosso estudo sobre continuidade sequencial fraca de polinômios. Definimos essa propriedade pontualmente, e vamos estudar a relação entre os polinômios fracamente sequencialmente contínuos na origem e aqueles que o são em todo o domínio. No capítulo 3, daremos prosseguimento a esse estudo. 
Definição 2.31. Um polinômio $P \in \mathcal{P}(E)$ é dito fracamente sequencialmente contínuo em $x \in E$ se para toda sequência $\left(x_{n}\right)_{n}$ convergindo fracamente para $x$ tivermos que $P\left(x_{n}\right) \rightarrow P(x)$. Se isto ocorrer para cada $x \in E$, diremos que $P$ é fracamente sequencialmente continuo.

Os subespaços de $P\left({ }^{N} E\right)$ formados pelos polinômios fracamente sequencialmente contínuos e pelos polinômios fracamente sequencialmente contínuos na origem são denotados respectivamente por $\mathcal{P}_{w s c}\left({ }^{N} E\right)$ e $\mathcal{P}_{w s c o}\left({ }^{N} E\right)$.

É facil ver que de fato $\mathcal{P}_{\text {wsc }}\left({ }^{N} E\right)$ e $\mathcal{P}_{\text {wsco }}\left({ }^{N} E\right)$ constituem subespaços de $\mathcal{P}\left({ }^{N} E\right)$. Vamos verificar que são subespaços fechados. Este fato é consequência da seguinte proposição:

Proposição 2.32. Seja $\left(P_{j}\right)_{j}$ uma sequência em $\mathcal{P}\left({ }^{N} E\right)$ convergindo a $P \in \mathcal{P}\left({ }^{N} E\right)$, e seja $x \in E$. Se para cada $j \in \mathbb{N}, P_{j}$ é fracamente sequencialmente contínuo em $x$, então $P$ é fracamente sequencialmente contínuo em $x$.

\section{Demonstração:}

Seja $\left(x_{n}\right)_{n}$ uma sequência convergindo fracamente a $x$. Temos que $P_{j}\left(x_{n}\right) \stackrel{n}{\rightarrow} P_{j}(x)$, $P_{j} \rightarrow P$ e existe $M>0$ tal que $\|x\|^{N} \leq M$ e $\left\|x_{n}\right\|^{N} \leq M \forall n \in \mathbb{N}$, já que $\left(x_{n}\right)_{n}$ é limitada em $E$. Como $P_{j} \rightarrow P$, existe $j_{o} \in \mathbb{N}$ tal que $\left\|P_{j_{o}}-P\right\| \leq \frac{\epsilon}{3 M}$ e $\left|P_{j_{o}}(x)-P(x)\right| \leq \frac{\epsilon}{3}$. Então

$$
\left|\left(P_{j_{o}}-P\right)\left(x_{n}\right)\right|=\left\|x_{n}\right\|^{N}\left|\left(P_{j_{o}}-P\right)\left(\frac{x_{n}}{\left\|x_{n}\right\|}\right)\right| \leq M \frac{\epsilon}{3 M}=\frac{\epsilon}{3},
$$

para todo $n \in \mathbb{N}$. Para esse cálculo consideramos $x_{n} \neq 0$, mas a desigualdade vale trivialmente quando $x_{n}=0$.

Já que $P_{j_{o}}\left(x_{n}\right) \rightarrow P_{j_{o}}(x)$, existe $n_{o}$ natural tal que para cada $n>n_{o}$ natural $\mid P_{j_{o}}\left(x_{n}\right)-$ $P_{j_{o}}(x) \mid \leq \frac{\epsilon}{3}$. Assim, para cada natural $n>n_{o}$

$\left|P\left(x_{n}\right)-P(x)\right| \leq\left|P\left(x_{n}\right)-P_{j_{o}}\left(x_{n}\right)\right|+\left|P_{j_{o}}\left(x_{n}\right)-P_{j_{o}}(x)\right|+\left|P_{j_{o}}(x)-P(x)\right| \leq \frac{\epsilon}{3}+\frac{\epsilon}{3}+\frac{\epsilon}{3}=\epsilon$.

Portanto, $P$ é fracamente sequencialmente contínuo em $x$.

Consequentemente temos:

Corolário 2.33. $\mathcal{P}_{w s c}\left({ }^{N} E\right)$ e $\mathcal{P}_{w s c 0}\left({ }^{N} E\right)$ são subespaços fechados de $\mathcal{P}\left({ }^{N} E\right)$.

Para estudar o próximo exemplo, bem como outros exemplos de polinômios em $l_{p}$, recordemos o seguinte resultado: 
Proposição 2.34. Sejam $p, q \in[1, \infty]$ tais que $p<q$ e $x \in l_{p}$. Então $x \in l_{q}$ e

$$
\|x\|_{q} \leq\|x\|_{p}
$$

Exemplo 2.35. Considero o espaço $l_{p}$, e seja $N \geq p$.

O polinômio $P \in \mathcal{P}\left({ }^{N} l_{p}\right)$ definido por $P(x) \doteq \sum_{n=1}^{\infty}\left(x_{n}\right)^{N}$ não é fracamente sequencialmente contínuo na origem.

Mostremos primeiro que $P$ está bem definido. Definindo para cada $i \in \mathbb{N} P_{i} \doteq$ $\sum_{n=1}^{i}\left(x_{n}\right)^{N}$, temos que cada $P_{i}$ é um elemento de $\mathcal{P}\left({ }^{N} E\right)$.

Pela proposição 2.34, para cada $x \in l_{p}$ a série $\sum_{n=1}^{\infty}\left(x_{n}\right)^{N}$ converge. Então para cada $x \in l_{p}$ temos que $P_{i}(x) \rightarrow P(x)$, e pelo Teorema de Banach-Steinhaus para polinômios (2.27) podemos concluir que $P \in \mathcal{P}\left({ }^{N} E\right)$.

Para verificar que $P$ não é fracamente sequencialmente contínuo na origem, basta observar que a sequência $e^{k}$ converge fracamente a 0 mas $P\left(e^{k}\right)=1$ para todo $k \in \mathbb{N}$, e desta forma $P\left(e^{k}\right) \not \rightarrow P(0)=0$.

É fundamental a condição $N \geq p$ para a existência de um polinômio homogêneo de grau $N$ que não é fracamente sequencialmente contínuo em $l_{p}$, de acordo com o seguinte resultado:

Teorema 2.36 (Teorema de Pitt). Seja $P \in \mathcal{P}\left({ }^{N} l_{p}\right)$, com $1<p<\infty$.

Se $N<p$, então $P$ é fracamente sequencialmente contínuo.

O Teorema acima decorre de um resultado de Pitt sobre aplicações bilineares que se encontra em [18]. Esse resultado posteriormente foi generalizado para aplicações multilineares quaisquer e polinômios homogêneos.

\subsection{Complexificação de polinômios}

$\operatorname{Seja~}(X,\|\cdot\|)$ um $\mathbb{R}$-espaço normado. Vamos definir em $X \times X$ as operações de soma de elementos de $X$ e de multiplicação de um elemento de $X$ por um escalar complexo da seguinte maneira: 
1) $\left(x_{1}, y_{1}\right)+\left(x_{2}, y_{2}\right) \doteq\left(x_{1}+x_{2}, y_{1}+y_{2}\right), \forall\left(x_{1}, y_{1}\right),\left(x_{2}, y_{2}\right) \in X \times X$

2) $(a+b i)(x, y) \doteq(a x-b y, b x+a y), \forall(a+b i) \in \mathbb{C}, \forall(x, y) \in X \times X$.

Verifica-se facilmente que $X \times X$ munido dessas operações é um $\mathbb{C}$-espaço vetorial, que iremos chamar de complexificação de $X$ e denotar por $X_{\mathbb{C}}{ }^{1}$

O raciocínio a seguir visa estabelecer uma notação análoga em $X_{\mathbb{C}}$ à que temos em $\mathbb{C}$. Seja $(x, y) \in X_{\mathbb{C}}$ e consideremos a equação de incógnitas $x_{1}$ e $x_{2}$ em $X$

$$
(x, y)=\left(x_{1}, 0\right)+i\left(x_{2}, 0\right) .
$$

Temos que $\left(x_{1}, 0\right)+i\left(x_{2}, 0\right)=\left(x_{1}, 0\right)+(0+1 i)\left(x_{2}, 0\right)=\left(x_{1}, 0\right)+\left(0, x_{2}\right)=\left(x_{1}, x_{2}\right)$, e desta forma a única solução dessa equação é $x_{1}=x$ e $x_{2}=y$. Podemos então concluir que $(x, y)$ se escreve de forma única como

$$
(x, y)=(x, 0)+i(y, 0) .
$$

Vamos definir $i X \doteq\{i y: y \in X\}$. iX é um $\mathbb{R}$-espaço vetorial normado se munido das operações naturais provenientes de $X$ e de norma $\|\cdot\|: i x \mapsto\|x\|$. Identificando $\{(x, 0): x \in X\} \operatorname{com} X$ e $\{i(y, 0): y \in X\} \operatorname{com} i X$, podemos tratar $X_{\mathbb{C}}$ como $X \oplus i X$, e escrever cada elemento $(x, y) \in X_{C}$ na forma $x+i y$ de maneira única.

Em $X_{\mathbb{C}}$ podemos introduzir a norma

$$
\|\cdot\|_{\mathbb{C}}: x+i y \mapsto\|x\|+\|y\| .
$$

É muito fácil verificar que $\|\cdot\|_{\mathbb{C}}$ é de fato uma norma sobre $X_{\mathbb{C}}$, de forma que vamos omitir esses cálculos.

Proposição 2.37. Se $(E,\|\cdot\|)$ é um $\mathbb{R}$-espaço de Banach, $\left(E_{\mathbb{C}},\|\cdot\|_{\mathbb{C}}\right)$ é um $\mathbb{C}$-espaço de Banach.

\section{Demonstração:}

Seja $\left(z_{n}\right)_{n}=\left(z_{n}^{0}+i z_{n}^{1}\right)_{n}$ uma sequência de Cauchy em $E_{\mathbb{C}}$, e verifiquemos que $\left(z_{n}\right)_{n}$ é convergente.

Vamos mostrar primeiro que as sequências $\left(z_{n}^{0}\right)_{n}$ e $\left(z_{n}^{1}\right)_{n}$ são convergentes em $E$ : seja $\epsilon>0$. Como $\left(z_{n}\right)_{n}$ é de Cauchy, existe $n_{o} \in \mathbb{N}$ tal que para cada $m, n \geq n_{o}$

$$
\left\|z_{n}^{0}-z_{m}^{0}\right\| \leq\left\|z_{n}^{0}-z_{m}^{0}\right\|+\left\|z_{n}^{1}-z_{m}^{1}\right\|=\left\|z_{n}^{0}-z_{m}^{0}+i\left(z_{n}^{1}-z_{m}^{1}\right)\right\|_{\mathbb{C}}=\left\|z_{n}-z_{m}\right\|_{\mathbb{C}}<\epsilon
$$

\footnotetext{
${ }^{1} \mathrm{O}$ espaço $X_{\mathbb{C}}$ também pode ser construido utilizando-se o conceito de produto tensorial, de forma bastante simplificada. Tem-se que $X_{\mathbb{C}}=X_{\mathbb{R}} \otimes_{\mathbb{R}} \mathbb{C}$.
} 
e portanto $\left(z_{n}^{0}\right)_{n}$ é de Cauchy em $E$. Analogamente, $\left(z_{n}^{1}\right)_{n}$ também é de Cauchy em $E$. Logo, como $E$ é espaço de Banach, existem em $E z^{0}$ e $z^{1}$ tais que $\left(z_{n}^{0}\right)_{n}$ converge a $z^{0}$ e $\left(z_{n}^{1}\right)_{n}$ converge a $z^{1}$.

Então $\left(z_{n}\right)_{n}$ converge a $\left(z^{0}+i z^{1}\right) \in E_{\mathbb{C}}$, já que

$$
\left\|z_{n}^{0}-z^{0}+i\left(z_{n}^{1}-z^{1}\right)\right\|=\left\|z_{n}^{0}-z^{0}\right\|+\left\|z_{n}^{1}-z^{1}\right\| \stackrel{n}{\rightarrow} 0 .
$$

O Teorema a seguir mostra como estender uma aplicação multilinear $A$ de um $\mathbb{R}$-espaço normado $X$ a uma aplicação multilinear $A_{\mathbb{C}}$ em $X_{\mathbb{C}}$, e as relações de continuidade entre $A$ e $A_{\mathbb{C}}$.

Teorema 2.38. Sejam $X$ um $\mathbb{R}$-espaço normado e $A \in \mathcal{L}_{a}\left({ }^{m} X\right)$. Então existe uma única aplicação $A_{\mathbb{C}} \in \mathcal{L}_{a}\left({ }^{m} X_{\mathbb{C}}\right)$ tal que $A_{\mathbb{C}}$ restrito a $X \times \cdots \times X$ é igual a $A$, isto é,

$$
A_{\mathbb{C}}\left(x_{1}, \ldots, x_{m}\right)=A\left(x_{1}, \ldots, x_{m}\right), \text { para cada } x_{1}, \ldots, x_{m} \in X
$$

$e A_{\mathbb{C}}$ é contínua se e somente se $A$ é contínua.

\section{Demonstração:}

Seja $A \in \mathcal{L}_{a}\left({ }^{m} X\right)$. Definimos $A_{\mathbb{C}}: X \times \cdots \times X \rightarrow \mathbb{C}$ da seguinte maneira:

$$
A_{\mathbb{C}}\left(x_{1}^{0}+i x_{1}^{1}, \ldots, x_{m}^{0}+i x_{m}^{1}\right) \doteq \sum_{\substack{\epsilon_{j}=0 \text { ou } 1 \\ 1 \leq j \leq m}} i^{\sum_{j} \epsilon_{j}} A\left(x_{1}^{\epsilon_{1}}, \ldots, x_{m}^{\epsilon_{m}}\right), \forall x_{1}^{0}+x_{1}^{1}, \ldots, x_{m}^{0}+i x_{m}^{1} \in X_{\mathbb{C}}
$$

Se $x_{1}^{1}=\cdots=x_{m}^{1}=0$, temos que $A\left(x_{1}^{\epsilon_{1}}, \ldots, x_{m}^{\epsilon_{m}}\right)=0$ sempre que $\epsilon_{j}=1$ para algum $j$, e desta forma $A_{\mathbb{C}}\left(x_{1}^{0}, \ldots, x_{m}^{0}\right)=A\left(x_{1}^{0}, \ldots, x_{m}^{0}\right)$. Podemos concluir então que que $A_{\mathbb{C}}$ restrito a $X \times \cdots \times X$ é igual a $A$.

Vamos mostrar que $A_{\mathbb{C}}$ é $m$-linear. Sejam $k \in\{1, \ldots, m\}, x_{1}^{0}+i x_{1}^{1}, \ldots, x_{m}^{0}+i x_{m}^{1}, y_{1}^{0}+$ $i y_{1}^{1}, \ldots, y_{m}^{0}+i y_{m}^{1} \in X_{\mathbb{C}}$ e $(a+b i) \in \mathbb{C}$. 
1)

$$
\begin{aligned}
& A_{\mathbb{C}}\left(x_{1}^{0}+i x_{1}^{1}, \ldots, x_{k}^{0}+i x_{k}^{1}+y_{k}^{0}+i y_{k}^{1}, \ldots, x_{m}^{0}+i x_{m}^{1}\right) \\
= & A_{\mathbb{C}}\left(x_{1}^{0}+i x_{1}^{1}, \ldots, x_{k}^{0}+y_{k}^{0}+i\left(x_{k}^{1}+y_{k}^{1}\right), \ldots, x_{m}^{0}+i x_{m}^{1}\right) \\
= & \sum_{\substack{\epsilon_{j}=0 \text { ou } 1 \\
i}} i^{\sum_{j} \epsilon_{j}} A\left(x_{1}^{\epsilon_{1}}, \ldots, x_{k}^{\epsilon_{k}}+y_{k}^{\epsilon_{k}}, \ldots, x_{m}^{\epsilon_{m}}\right) \\
= & \sum_{\substack{\epsilon_{j}=0 \text { ou } 1 \\
1 \leq j \leq m}} i^{\sum_{j} \epsilon_{j}} A\left(x_{1}^{\epsilon_{1}}, \ldots, x_{k}^{\epsilon_{k}}, \ldots, x_{m}^{\epsilon_{m}}\right)+\sum_{\substack{\epsilon_{j}=0 \text { ou } 1 \\
1 \leq j \leq m}} i^{\sum_{j} \epsilon_{j}} A\left(x_{1}^{\epsilon_{1}}, \ldots, y_{k}^{\epsilon_{k}}, \ldots, x_{m}^{\epsilon_{m}}\right) \\
= & A_{\mathbb{C}}\left(x_{1}^{0}+i x_{1}^{1}, \ldots, x_{k}^{0}+i x_{k}^{1}, \ldots, x_{m}^{0}+i x_{m}^{1}\right)+A_{\mathbb{C}}\left(x_{1}^{0}+i x_{1}^{1}, \ldots, y_{k}^{0}+i y_{k}^{1}, \ldots, x_{m}^{0}+i x_{m}^{1}\right) ;
\end{aligned}
$$

2)

$$
\begin{aligned}
& A_{\mathbb{C}}\left(x_{1}^{0}+i x_{1}^{1}, \ldots,(a+b i)\left(x_{k}^{0}+i x_{k}^{1}\right), \ldots, x_{m}^{0}+i x_{m}^{1}\right) \\
& =A_{\mathbb{C}}\left(x_{1}^{0}+i x_{1}^{1}, \ldots, a x_{k}^{0}-b x_{k}^{1}+i\left(b x_{k}^{0}+a x_{k}^{1}\right), \ldots, x_{m}^{0}+i x_{m}^{1}\right) \\
& =\sum_{\epsilon_{j}=0 \text { ou } 1} i^{\sum_{j \neq k} \epsilon_{j}} A\left(x_{1}^{\epsilon_{1}}, \ldots, a x_{k}^{0}-b x_{k}^{1}, \ldots, x_{m}^{\epsilon_{m}}\right) \\
& j \neq k \\
& +\sum i^{1+\sum_{j \neq k} \epsilon_{j}} A\left(x_{1}^{\epsilon_{1}}, \ldots, b x_{k}^{0}+a x_{k}^{1}, \ldots, x_{m}^{\epsilon_{m}}\right) \\
& \epsilon_{j}=0 \text { ou } 1 \\
& j \neq k \\
& \begin{array}{c}
=a \sum_{\substack{\epsilon_{j}=0 \text { ou } 1 \\
j \neq k}} i^{\sum_{j \neq k} \epsilon_{j}} A\left(x_{1}^{\epsilon_{1}}, \ldots, x_{k}^{0}, \ldots, x_{m}^{\epsilon_{m}}\right)-b \sum_{\substack{\epsilon_{j}=0 \text { ou } 1 \\
j \neq k}} i^{\sum_{j \neq k} \epsilon_{j}} A\left(x_{1}^{\epsilon_{1}}, \ldots, x_{k}^{1}, \ldots, x_{m}^{\epsilon_{m}}\right) \\
+b \sum_{\substack{\epsilon_{j}=0 \text { ou } 1 \\
j \neq k}} i^{1+\sum_{j \neq k} \epsilon_{j}} A\left(x_{1}^{\epsilon_{1}}, \ldots, x_{k}^{0}, \ldots, x_{m}^{\epsilon_{m}}\right)+a \sum_{\substack{\epsilon_{j}=0 \text { ou } 1 \\
j \neq k}} i^{1+\sum_{j \neq k} \epsilon_{j}} A\left(x_{1}^{\epsilon_{1}}, \ldots, x_{k}^{1}, \ldots, x_{m}^{\epsilon_{m}}\right)
\end{array} \\
& =(a+b i) \sum_{\substack{\epsilon_{j}=0 \text { ou } 1 \\
j \neq k}} i^{\sum_{j \neq k} \epsilon_{j}} A\left(x_{1}^{\epsilon_{1}}, \ldots, x_{k}^{0}, \ldots, x_{m}^{\epsilon_{m}}\right) \\
& +(-b+a i) \sum_{\substack{\epsilon_{j}=0 \text { ou } 1 \\
j \neq k}} i^{\sum_{j \neq k} \epsilon_{j}} A\left(x_{1}^{\epsilon_{1}}, \ldots, x_{k}^{1}, \ldots, x_{m}^{\epsilon_{m}}\right) \\
& =(a+b i) \sum_{\substack{\epsilon_{j}=0 \text { ou } 1 \\
j \neq k}} i^{\sum_{j \neq k} \epsilon_{j}} A\left(x_{1}^{\epsilon_{1}}, \ldots, x_{k}^{0}, \ldots, x_{m}^{\epsilon_{m}}\right) \\
& +(a+b i) \sum_{\substack{\epsilon_{j}=0 \text { ou } 1 \\
j \neq k}} i^{1+\sum_{j \neq k} \epsilon_{j}} A\left(x_{1}^{\epsilon_{1}}, \ldots, x_{k}^{1}, \ldots, x_{m}^{\epsilon_{m}}\right)
\end{aligned}
$$




$$
\begin{aligned}
& =(a+b i) \sum_{\substack{\epsilon_{j}=0 \text { ou } 1 \\
1 \leq j \leq m}} i^{\sum_{j} \epsilon_{j}} A\left(x_{1}^{\epsilon_{1}}, \ldots, x_{k}^{\epsilon_{k}}, \ldots, x_{m}^{\epsilon_{m}}\right) \\
& =(a+b i) A_{\mathbb{C}}\left(x_{1}^{0}+i x_{1}^{1}, \ldots, x_{k}^{0}+i x_{k}^{1}, \ldots, x_{m}^{0}+i x_{m}^{1}\right) .
\end{aligned}
$$

Pela maneira que $A_{\mathbb{C}}$ foi definida, temos que se $A$ é contínua então $A_{\mathbb{C}}$ também é. Reciprocamente, se $A_{\mathbb{C}}$ for contínua, $A$ também será por ser uma restrição de $A_{\mathbb{C}}$.

Corolário 2.39. Sejam $X$ um $\mathbb{R}$-espaço vetorial e $P \in \mathcal{P}_{a}\left({ }^{m} X\right)$. Então existe uma única aplicação $P_{\mathbb{C}} \in \mathcal{P}_{a}\left({ }^{m} X_{\mathbb{C}}\right)$ tal que $P_{\mathbb{C}}$ restrito a $X$ é igual a $P$, isto é,

$$
P_{\mathbb{C}}(x)=P(x), \text { para cada } x \in X,
$$

e $P_{\mathbb{C}}$ é contínuo se e somente se $P$ é contínuo.

$P_{\mathbb{C}}$ é chamado de complexificação de $P$.

\section{Demonstração:}

Pela proposição 2.24, $P$ está associado biunivocamente a uma aplicação $A \in \mathcal{L}_{a s}\left({ }^{m} X\right)$, de forma que para cada $x \in X, P(x)=A x^{m}$. Pelo Teorema 2.38, existe uma única aplicação $A_{\mathbb{C}} \in \mathcal{L}_{a}\left({ }^{m} X_{\mathbb{C}}\right)$ tal que para cada $x_{1}, \ldots, x_{m} \in X, A_{\mathbb{C}}\left(x_{1}, \ldots, x_{m}\right)=A\left(x_{1}, \ldots, x_{m}\right)$. E novamente pela proposição 2.24 existe um único polinômio $P_{\mathbb{C}} \in \mathcal{P}_{a}\left({ }^{m} X_{\mathbb{C}}\right)$ tal que para cada $x \in X, P_{\mathbb{C}}(x)=A_{\mathbb{C}} x^{m}$. Então $P_{\mathbb{C}}$ é de fato o único polinômio de $\mathcal{P}_{a}\left({ }^{m} X_{\mathbb{C}}\right)$ tal que $P_{\mathbb{C}}(x)=P(x), \forall x \in X$.

Além disso, pela proposição 2.24 as relações biunívocas entre $A$ e $P$ e entre $A_{\mathbb{C}}$ e $P_{\mathbb{C}}$ preservam continuidade,e pelo Teorema $2.38 A$ é contínua se e somente se $A_{\mathbb{C}}$ o é. Portanto, $P_{\mathbb{C}}$ é contínuo se e somente se $P$ o é.

Observação: De acordo com a definição de $A_{\mathbb{C}}, P_{\mathbb{C}}$ será escrito da seguinte forma em função de $P$ :

$$
P_{\mathbb{C}}\left(x^{0}+i x^{1}\right)=\sum_{\substack{\epsilon_{j}=0 \text { ou } 1 \\ 1 \leq j \leq m}} i^{\sum_{j} \epsilon_{j}} \check{P}\left(x^{\epsilon_{1}}, \ldots, x^{\epsilon_{m}}\right), \forall x^{0}+i x^{1} \in X_{\mathbb{C}}
$$




\section{Capítulo 3}

\section{O conjunto $C_{P}$}

Este capítulo é dedicado ao estudo do conjunto de continuidade sequencial fraca de um dado polinômio de $\mathcal{P}\left({ }^{N} E\right)$. Os resultados aqui colocados foram obtidos por R. Aron e V. Dimant em [2]. Na Seção 3.1 realizamos um estudo das propriedades gerais deste conjunto e algumas consequências interessantes quando $E$ é um espaço de Banach qualquer. Também analisamos alguns exemplos e questionamentos naturais acerca de tais conjuntos.

$\mathrm{Na}$ Seção seguinte são analizados os resultados e questionamentos no caso em que o espaço $E$ satisfaz certas condições adicionais, a saber, separabilidade e presença de FDD incondicional.

O exemplo a seguir motiva o estudo do conjunto de continuidade sequencial fraca de um dado polinômio:

Exemplo 3.1. Seja $P \in \mathcal{P}\left({ }^{3} l_{2}\right)$ definido para cada $x=\left(x_{n}\right)_{n} \in l_{2}$ por $P(x)=x_{1} \sum_{n=1}^{\infty} x_{n}^{2}$.

Então $P$ é fracamente sequencialmente contínuo em $x$ se e somente se $x_{1}=0$.

Seja $x \in l_{2}$ com $x_{1}=0$ e seja $\left(x^{k}\right)_{k}$ uma sequência em $l_{2}$ tal que $x^{k} \stackrel{w}{\rightarrow} x$. Vamos mostrar que $\left|P\left(x^{k}\right)-P(x)\right| \rightarrow 0$.

$\left|P\left(x^{k}\right)-P(x)\right|=\left|x_{1}^{k} \sum_{n=1}^{\infty}\left(x_{n}^{k}\right)^{2}-x_{1} \sum_{n=1}^{\infty} x_{n}^{2}\right|=\left|x_{1}^{k} \sum_{n=1}^{\infty}\left(x_{n}^{k}\right)^{2}\right|=\left|x_{1}^{k}\right|\left|\sum_{n=1}^{\infty}\left(x_{n}^{k}\right)^{2}\right|$.

Pelo corolário 1.34 temos que $x_{1}^{k} \stackrel{k}{\rightarrow} x_{1}=0$ e que existe $M \geq 0$ tal que $\left|\sum_{i=1}^{\infty}\left(x_{i}^{k}\right)^{2}\right| \leq$ $\left\|x^{k}\right\|_{2}^{2} \leq M$, para todo $k$ natural, portanto $\left|P\left(x^{k}\right)-P(x)\right|=\left|x_{1}^{k}\right|\left|\sum_{n=1}^{\infty}\left(x_{n}^{k}\right)^{2}\right| \leq\left|x_{1}^{k}\right| M \rightarrow 0$, como queríamos. 
Para mostrarmos a outra implicação, basta mostrar que, se $x=\left(x_{n}\right)_{n} \in l_{2}$ é tal que $x_{1} \neq 0$, então existe uma sequência $\left(x^{k}\right)_{k}$ em $l_{2}$ convergindo fracamente a $x$ tal que $P\left(x^{k}\right) \not \rightarrow P(x)$. Seja $\left(x^{k}\right)$ definida por $x^{k} \doteq\left(x_{1}, \ldots, x_{k-1}, x_{k}+1, x_{k+1}, \ldots\right)$. Então $x^{k} \stackrel{w}{\rightarrow} x$ e para $k>1$ temos que

$$
\begin{aligned}
\left|P\left(x^{k}\right)-P(x)\right| & =\left|x_{1}^{k} \sum_{n=1}^{\infty}\left(x_{n}^{k}\right)^{2}+x_{1} \sum_{n=1}^{\infty}\left(x_{n}\right)^{2}\right| \\
& =\left|x_{1}\left(\sum_{n \neq k}\left(x_{n}\right)^{2}+\left(x_{k}+1\right)^{2}-\sum_{n=1}^{\infty}\left(x_{n}\right)^{2}\right)\right| \\
& =\left|x_{1}\left(\sum_{n \neq k}\left(x_{n}\right)^{2}+\left(x_{k}\right)^{2}+2 x_{k}+1-\sum_{n=1}^{\infty}\left(x_{n}\right)^{2}\right)\right| \\
& =\left|x_{1}\left(2 x_{k}+1\right)\right| .
\end{aligned}
$$

Consideremos $\epsilon \in] 0,\left|x_{1}\right|\left[\right.$. Sabemos que $x_{k} \stackrel{k}{\rightarrow} 0$, e assim $\left|P\left(x^{k}\right)-P(x)\right|=\mid x_{1}\left(2 x_{k}+\right.$ $1)|\rightarrow| x_{1} \mid>\epsilon$. Portanto, $P\left(x^{k}\right) \not \rightarrow P(x)$, como queríamos.

Definição 3.2. Seja $P \in \mathcal{P}(E)$. Denotamos por $C_{P}$ o conjunto $\{x \in E: P$ é fracamente sequencialmente contínuo em $x\}$.

Embora a definição do conjunto $C_{P}$ abrange polinômios contínuos quaisquer, vamos focalizar nosso estudo em polinômios homogêneos.

Observe que para cada espaço de Banach $E$ onde todos os polinômios são fracamente sequencialmente contínuos, teremos que todo polinômio homogêneo contínuo $P$ nesse espaço satisfará $C_{P}=E$. Desta forma, não estudaremos os conjuntos do tipo $C_{P}$ nesses espaços, embora vale notar que a existência de espaços assim caracterizados motivou o estudo dos conjuntos do tipo $C_{P}$. Exemplos de espaços em que todo polinômio é fracamente sequencialmente contínuo são os espaços de dimensão finita e espaços com a propriedade de Dunford-Pettis ${ }^{1}$.

\footnotetext{
${ }^{1}$ Dizemos que $E$ satisfaz a propriedade de Dunford-Pettis se para toda $\left(x_{n}\right)_{n}$ sequência fracamente convergente a 0 em $E$ e toda sequência $\left(x_{n}^{*}\right)_{n}$ fracamente convergente a aplicação nula em $E^{\prime}$ tivermos que $\lim _{n \rightarrow \infty} x_{n}^{*}\left(x_{n}\right)=0$. Outras caracterizações desse tipo de espaço se encontram em [12]. Ryan mostrou em 1979 (ver [20]) que a proriedade de Dunford-Pettis é equivalente à propriedade de Dunford-Pettis polinomial, e deste resultado pode-se concluir que todos os polinômios contínuos em $E$ são fracamente sequencialmente contínuos.
} 
Vamos estudar primeiro o conjunto $C_{P}$ no caso em que $P$ é um polinômio 2-homogêneo:

Proposição 3.3. Se $P \in \mathcal{P}\left({ }^{2} E\right)$ é fracamente sequencialmente contínuo em 0 , então $P$ é fracamente sequencialmente contínuo em $E$. Isto é, $\mathcal{P}_{\text {wsc }}\left({ }^{2} E\right)=\mathcal{P}_{\text {wsco }}\left({ }^{2} E\right)$.

\section{Demonstração:}

Sejam $P \in \mathcal{P}_{\text {wsc } 0}\left({ }^{2} E\right)$ e $x \in E$. Seja $\left(x_{n}\right)_{n}$ sequência em $E$ tal que $x_{n} \stackrel{w}{\rightarrow} x$. Mostremos que $P\left(x_{n}\right)-P(x) \rightarrow 0$.

$P\left(x_{n}\right)-P(x)=\check{P}\left(x_{n}, x_{n}\right)-\check{P}(x, x)=\check{P}\left(x_{n}-x, x_{n}\right)+\check{P}\left(x, x_{n}-x\right) \mathrm{e}$

$P\left(x_{n}-x\right)=\check{P}\left(x_{n}-x, x_{n}-x\right)=\check{P}\left(x_{n}-x, x_{n}\right)-\check{P}\left(x_{n}-x, x\right)$.

Sendo $\check{P}$ simétrica,

$P\left(x_{n}\right)-P(x)=P\left(x_{n}-x\right)+2 \check{P}\left(x_{n}-x, x\right)$.

Como $x_{n}-x \stackrel{w}{\rightarrow} 0$ e $P$ é fracamente sequencialmente contínuo em 0 , temos que $P\left(x_{n}-\right.$ $x) \rightarrow 0$.

Temos que $2 \check{P}(\cdot, x) \in E^{\prime}$ e $x_{n}-x \stackrel{w}{\rightarrow} 0$, $\log 2 \check{P}\left(x_{n}-x, x\right) \rightarrow 0$.

Portanto $P\left(x_{n}\right)-P(x)=P\left(x_{n}-x\right)+2 \check{P}\left(x_{n}-x, x\right) \rightarrow 0$, como queríamos.

Vemos pelo exemplo 3.1 que não temos resultado análogo para polinômios de grau 3.

\subsection{Propriedades gerais de $C_{P}$}

Seja $P \in \mathcal{P}\left({ }^{N} E\right)$. Vamos definir para cada $j \in\{0, \ldots, N\}$ e cada $x \in E$ o polinômio $\phi_{j}(x) \in \mathcal{P}\left({ }^{N-j} E\right)$ por $\phi_{j}(x)(y) \doteq \check{P} x^{j} y^{N-j}$. Observemos que $\phi_{j}$ é um polinômio $j$-homogêneo contínuo de $E$ em $\mathcal{P}\left({ }^{N-j} E\right)$, associado à aplicação multilinear simétrica $A \in \mathcal{L}_{s}\left({ }^{j} E ; P\left({ }^{N-j} E\right)\right)$ definida por $A\left(x_{1}, \ldots, x_{j}\right)(y)=\check{P} x_{1} \ldots x_{j} y^{N-j}$.

Estabelecido esse conceito, vamos iniciar nosso estudo sobre as propriedades gerais de $C_{P}$.

Proposição 3.4. Seja $P \in \mathcal{P}\left({ }^{N} E\right)$. Então:

1) $C_{P}$ é fechado;

2) Se $x \in C_{P}$, então $\lambda x \in C_{P}, \forall \lambda \in \mathbb{K}$. Em particular, se $C_{P} \neq \emptyset$, então $0 \in C_{P}$.

3) $C_{P}=\cap_{j=0}^{N-2}\left\{x \in E \mid \phi_{j}(x) \in \mathcal{P}_{w s c 0}\left({ }^{N-j} E\right)\right\}$.

\section{Demonstração:}


(1) Seja $x \in E$ e $\left(y_{n}\right)_{n}$ sequência em $C_{P}$ convergindo em norma para $x$. Precisamos mostrar que $x \in C_{P}$ : para tal, seja $\left(x_{n}\right)_{n}$ sequência em $E$ tal que $x_{n} \stackrel{w}{\rightarrow} x$, e vamos provar que $P\left(x_{n}\right) \rightarrow P(x)$.

Seja $\epsilon>0$. Como $y_{n}$ converge a $x$, então existe $j_{o} \in \mathbb{N}$ tal que $\left\|y_{j_{o}}-x\right\|<\epsilon$. Como $x_{n} \stackrel{w}{\rightarrow} x$, segue que $y_{j_{o}}+\left(x_{n}-x\right) \stackrel{w}{\rightarrow} y_{j_{o}}$ e pelo fato de $y_{j_{o}} \in C_{P}$, temos que $P\left(y_{j_{o}}+\left(x_{n}-x\right)\right) \rightarrow P\left(y_{j_{o}}\right)$. Logo, existe $n_{o} \in \mathbb{N}$ tal que $\left|P\left(y_{j_{o}}+\left(x_{n}-x\right)\right)-P\left(y_{j_{o}}\right)\right|<\epsilon$, para cada $n>n_{o}$.

Para cada $n>n_{o}$ natural então

$\left|P\left(x_{n}\right)-P(x)\right| \leq\left|P\left(x_{n}\right)-P\left(y_{j_{o}}+\left(x_{n}-x\right)\right)\right|+\left|P\left(y_{j_{o}}+\left(x_{n}-x\right)\right)-P\left(y_{j_{o}}\right)\right|+\left|P\left(y_{j_{o}}\right)-P(x)\right|$.

Como as sequências $\left(x_{n}\right)_{n},\left(y_{n}\right)_{n}$ e $\left(y_{j_{o}}+\left(x_{n}-x\right)\right)_{n}$ são fracamente convergentes, são limitadas pela proposição 1.32. Então pela proposição 2.22 existe $M \geq 0$ tal que para todo $u, v \in\left\{x_{n}: n \in \mathbb{N}\right\} \cup\left\{y_{n}: n \in \mathbb{N}\right\} \cup\left\{y_{j_{o}}+\left(x_{n}-x\right): n \in \mathbb{N}\right\} \cup\{x\}$ temos que $|P(u)-P(v)| \leq M\|u-v\|$. Desta forma, para cada $n \geq n_{o}$ natural

$$
\begin{aligned}
& \left|P\left(x_{n}\right)-P\left(y_{j_{o}}+\left(x_{n}-x\right)\right)\right| \leq M\left\|x_{n}-y_{j_{o}}-\left(x_{n}-x\right)\right\|=M\left\|x-y_{j_{o}}\right\| \leq M \epsilon ; \\
& \left|P\left(y_{j_{o}}+\left(x_{n}-x\right)\right)-P\left(y_{j_{o}}\right)\right| \leq \epsilon \mathrm{e} \\
& \left|P\left(y_{j_{o}}\right)-P(x)\right| \leq M\left\|y_{j_{o}}-x\right\| \leq M \epsilon .
\end{aligned}
$$

Logo, $\left|P\left(x_{n}\right)-P(x)\right| \leq(2 M+1) \epsilon, \forall n \geq n_{o}$ natural, como queríamos.

(2) Seja $x \in C_{P}$ e $\lambda \in \mathbb{K}$ não nulo, e vamos mostrar que $\lambda x \in C_{P}$.

Seja $\left(x_{n}\right)_{n}$ uma sequência tal que $x_{n} \stackrel{w}{\rightarrow} \lambda x$. Como $x \in C_{P}$ e $\frac{x_{n}}{\lambda} \stackrel{w}{\rightarrow} x$, então $\frac{1}{\lambda^{N}} P\left(x_{n}\right)=$ $P\left(\frac{x_{n}}{\lambda}\right) \rightarrow P(x)$, e logo $P\left(x_{n}\right) \rightarrow \lambda^{N} P(x)=P(\lambda x)$, como queríamos.

Sendo o resultado válido para cada $\lambda \neq 0$, e como $\frac{1}{n} x \rightarrow 0$, pelo item (1) temos que $0 \in C_{P}$. Portanto, $\lambda x \in C_{P}, \forall \lambda \in \mathbb{K}$.

(3) Seja $x \in \cap_{j=0}^{N-2}\left\{x \in E: \phi_{j}(x) \in \mathcal{P}_{w s c 0}\left({ }^{N-j} E\right)\right\}$, e mostremos que $x \in C_{P}$. Para tal, seja $\left(x_{n}\right)_{n}$ sequência convergindo fracamente a $x$. Pela fórmula binomial (2.13),

$$
P\left(x_{n}\right)=P\left(x+\left(x_{n}-x\right)\right)=\sum_{j=0}^{N}\left(\begin{array}{c}
N \\
j
\end{array}\right) \check{P} x^{j}\left(x_{n}-x\right)^{N-j} .
$$

Para cada $j \in\{1, \ldots, N-2\}, \phi_{j}(x)$ é fracamente sequencialmente contínuo na origem, $\operatorname{logo}\left(\begin{array}{c}N \\ j\end{array}\right) \check{P} x^{j}\left(x_{n}-x\right)^{N-j}=\left(\begin{array}{c}N \\ j\end{array}\right) \phi_{j}(x)\left(x_{n}-x\right) \stackrel{n}{\rightarrow} 0$. A aplicação $y \mapsto\left(\begin{array}{c}N \\ N-1\end{array}\right) \check{P} x^{N-1} y$ é linear, de forma que $\left(\begin{array}{c}N \\ N-1\end{array}\right) \check{P} x^{j}\left(x_{n}-x\right) \rightarrow 0$. Desta forma, obtemos que $P\left(x_{n}\right) \rightarrow$ 
$\left(\begin{array}{l}N \\ N\end{array}\right) \check{P} x^{N}\left(x_{n}-x\right)^{0}=P(x)$, como queríamos.

Seja agora $x \in C_{P}$, e vamos mostrar que, para cada $j \in\{0, \ldots, N-2\}, \phi_{j}(x)$ é fracamente sequencialmente contínuo na origem. Pelo item anterior, basta mostrar que $x \in C_{\phi_{j}(x)}$. Fixemos então $j \in\{0, \ldots, N-2\}$ e seja $\left(x_{n}\right)_{n}$ uma sequência tal que $x_{n} \stackrel{w}{\rightarrow} x$.

Pela fórmula de polarização (2.14),

$$
\phi_{j}(x)\left(x_{n}\right)=\check{P} x^{j} x_{n}^{N-j}=\frac{1}{N ! 2^{N}} \sum_{\substack{\epsilon_{i}= \pm 1 \\ 1 \leq i \leq N}} \epsilon_{1} \ldots \epsilon_{N} P\left(\left(\epsilon_{1}+\cdots+\epsilon_{j}\right) x+\left(\epsilon_{j+1}+\cdots+\epsilon_{N}\right) x_{n}\right) \text {. }
$$

Como $\left(\epsilon_{1}+\cdots+\epsilon_{j}\right) x+\left(\epsilon_{j+1}+\cdots+\epsilon_{N}\right) x_{n} \stackrel{w}{\rightarrow}\left(\epsilon_{1}+\cdots+\epsilon_{N}\right) x$ e, pelo item anterior, $\left(\epsilon_{1}+\cdots+\epsilon_{N}\right) x \in C_{P}$, segue que $P\left(\left(\epsilon_{1}+\cdots+\epsilon_{j}\right) x+\left(\epsilon_{j+1}+\cdots+\epsilon_{N}\right) x_{n}\right) \rightarrow P\left(\left(\epsilon_{1}+\cdots+\right.\right.$ $\left.\left.\epsilon_{N}\right) x\right), \forall \epsilon_{i}= \pm 1, \forall i \in\{1, \ldots, N\}$. Assim,

$$
\phi_{j}(x)\left(x_{n}\right) \stackrel{n}{\rightarrow} \frac{1}{N ! 2^{N}} \sum_{\substack{\epsilon_{i}= \pm 1 \\ 1 \leq i \leq N}} \epsilon_{1} \ldots \epsilon_{N} P\left(\left(\epsilon_{1}+\cdots+\epsilon_{j}\right) x\right)=P(x)=\phi_{j}(x)(x) .
$$

Observações: O item (1) também é válido para polinômios de $\mathcal{P}(E)$ (não necessariamente homogêneos). A demonstração é a mesma, exceto onde usamos a proposição 2.22; aí usamos a proposição 2.29 .

O item (2) não é válido para polinômios quaisquer de $\mathcal{P}(E)$ : se considerarmos em $l_{p}$ $(1<p<\infty)$ o polinômio $P$ definido para cada $x=\left(x_{n}\right)_{n} \in l_{p}$ por

$$
P(x) \doteq\left(\left(x_{1}\right)^{2}-1\right) \sum_{n=1}^{\infty}\left(x_{n}\right)^{2}
$$

usando uma técnica semelhante à usada no exemplo 3.1 mostramos que $C_{P}=\left\{\left(x_{n}\right)_{n} \in\right.$ $l_{p}: x_{1}=1$ ou $\left.x_{1}=-1\right\}$. Portanto (2) não é válida para este polinômio.

Corolário 3.5. Se E é um espaço de Banach tal que $\mathcal{P}\left({ }^{i} E\right)=\mathcal{P}_{w s c}\left({ }^{i} E\right), \forall i \in\{1, \ldots, r-1\}$ e $\mathcal{P}\left({ }^{r} E\right) \neq \mathcal{P}_{\text {wsc }}\left({ }^{r} E\right)$, então valem as seguintes afirmações:

1) $\forall P \in \mathcal{P}\left({ }^{r} E\right)$, ou $C_{P}=\emptyset$ ou $C_{P}=E$;

2) $\forall P \in \mathcal{P}\left({ }^{r+1} E\right)$, ou $C_{P}=\emptyset$ ou $C_{P}$ é um subespaço de $E$.

\section{Demonstração}

(1) Pelo item (3) da proposição acima, $C_{P}=\cap_{j=0}^{r-2}\left\{x \in E: \phi_{j}(x) \in \mathcal{P}_{w s c 0}\left({ }^{r-j} E\right)\right\}$. Por hipótese, para cada $x \in E, \phi_{j}(x) \in \mathcal{P}_{w s c}\left({ }^{r-j} E\right), \forall j \in\{1, \ldots, r-2\}$. Assim $\{x \in E$ : $\left.\phi_{j}(x) \in \mathcal{P}_{w s c 0}\left({ }^{r-j} E\right)\right\}=E, \forall j \in\{1, \ldots, r-2\}$. 
Resta mostrar então que $\left\{x \in E: \phi_{0}(x) \in \mathcal{P}_{w s c 0}\left({ }^{r} E\right)\right\}=\emptyset$ ou $E$. Mas $\phi_{0}(x)(y) \doteq$ $\check{P} x^{0} y^{r}=P(y)$, isto é, $\phi_{0}(x) \equiv P, \forall x \in E$. Portanto, se $P$ é fracamente sequencialmente contínuo na origem, $\left\{x \in E: \phi_{0}(x) \in \mathcal{P}_{w s c 0}\left({ }^{r} E\right)\right\}=E$, e caso contrário $\left\{x \in E: \phi_{0}(x) \in\right.$ $\left.\mathcal{P}_{\text {wsc } 0}\left({ }^{r} E\right)\right\}=\emptyset$, como queríamos.

(2) Como $P$ é $(\mathrm{r}+1)$-homogêneo, agora $C_{P}=\cap_{j=0}^{r-1}\left\{x \in E: \phi_{j}(x) \in \mathcal{P}_{w s c 0}\left({ }^{r+1-j} E\right)\right\}$. Usando o mesmo raciocínio do item (1), $\left\{x \in E: \phi_{j}(x) \in \mathcal{P}_{w s c 0}\left({ }^{r+1-j} E\right)\right\}=E$ para cada $j \in\{2, \ldots, r-1\}$ e $\left\{x \in E: \phi_{0}(x) \in \mathcal{P}_{w s c 0}\left({ }^{r+1} E\right)\right\}=\emptyset$ ou $E$. Assim, basta mostrar que $\left\{x \in E \mid \phi_{1}(x) \in \mathcal{P}_{w s c 0}\left({ }^{r} E\right)\right\}=\phi_{1}^{-1}\left(\mathcal{P}_{w s c 0}\left({ }^{r} E\right)\right)$ é um subespaço de $E$.

Mas $\mathcal{P}_{\text {wsc } 0}\left({ }^{r} E\right)$ é um subespaço de $\mathcal{P}\left({ }^{r} E\right)$ e $\phi_{j} \in \mathcal{L}\left(E ; \mathcal{P}\left({ }^{r} E\right)\right)$, e assim podemos concluir que $\phi_{1}^{-1}\left(\mathcal{P}_{\text {wsco }}\left({ }^{r} E\right)\right)$ é um subespaço por ser imagem inversa de subespaço por transformação linear.

Observe que, se $E=l_{p}$ e $N$ é o menor inteiro $\geq p$, pelo Teorema de Pitt (2.36) todo polinômio de grau $\leq N-1$ é fracamente sequencialmente contínuo, e que o polinômio $P \in \mathcal{P}\left({ }^{N} l_{p}\right)$ definido por $P(x) \doteq \sum_{i=1}^{\infty}\left(x_{i}\right)^{N}$ não é fracamente sequencialmente contínuo, já que $e^{k} \stackrel{w}{\rightarrow} 0$ mas $P\left(e^{k}\right) \not \nrightarrow 0$. Portanto esse espaço está nas hipóteses do corolário, com $r=N$.

Na demonstração da próxima proposição vamos utilizar o seguinte lema:

Lema 3.6. Seja $P \in \mathcal{P}\left({ }^{r} E ; F\right)$. Se $\left\{\gamma_{1}, \ldots, \gamma_{r+1}\right\} \subset E^{\prime}$ é um conjunto linearmente independente e $S$ é um subespaço de $F$ tais que $P\left(\gamma_{j}^{-1}(0)\right) \subset S$ para cada $j \in\{1, \ldots, r+1\}$, então $P(E) \subset S$.

\section{Demonstração:}

Demonstremos por indução. Provemos inicialmente que o resultado é válido para $r=1$ : neste caso, $P$ é linear e $\left\{\gamma_{1}, \gamma_{2}\right\} \subset E^{\prime}$ é um conjunto linearmente independente com $P\left(\gamma_{1}^{-1}(0)\right) \subset S$ e $P\left(\gamma_{2}^{-1}(0)\right) \subset S$. Observe que $\gamma_{1}^{-1}(0)$ e $\gamma_{2}^{-1}(0)$ são hiperplanos de $E$, distintos devido à independência linear de $\left\{\gamma_{1}, \gamma_{2}\right\}$. Existe portanto $v \in \gamma_{2}^{-1}(0) \backslash \gamma_{1}^{-1}(0) \mathrm{e}$ temos que $E=\gamma_{1}^{-1}(0)+[v]$.

Seja $x \in E$, e mostremos que $P(x) \in S$. Pela decomposição acima, $x$ se escreve como $x=x_{1}+x_{2}$, onde $x_{1} \in \gamma_{1}^{-1}(0)$ e $x_{2} \in[v] \subset \gamma_{2}^{-1}(0)$. Como $P$ é linear, $P(x)=$ $P\left(x_{1}+x_{2}\right)=P\left(x_{1}\right)+P\left(x_{2}\right)$. Por hipótese, $P\left(x_{1}\right)$ e $P\left(x_{2}\right) \in S$ e $S$ é subespaço, e portanto $P\left(x_{1}\right)+P\left(x_{2}\right) \in S$. 
Suponhamos agora que o resultado é válido para todo $k \leq r-1$, e mostremos que valerá também para $k=r$. Seja então $x \in E$ e consideremos as hipóteses tais como estão enunciadas no lema. Mostremos que $P(x) \in S$. Observemos inicialmente que pela proposição $1.9, \cap_{i=2}^{r+1} \gamma_{i}^{-1}(0)$, só poderia estar contido em $\gamma_{1}^{-1}(0)$ se existissem $\alpha_{2}, \ldots, \alpha_{r+1}$ tais que $\gamma_{1}=\sum_{i=2}^{r+1} \alpha_{i} \gamma_{i}$ e isto não ocorre por hipótese, então existe $v \in\left(\cap_{i=2}^{r+1} \gamma_{i}^{-1}(0)\right) \backslash \gamma_{1}^{-1}(0)$ e como no caso $r=1$ temos que $E=\gamma_{1}^{-1}(0)+[v]$. Desta forma, $x$ se escreve como $x=x_{1}+e$, onde $x_{1} \in \gamma_{1}^{-1}(0)$ e $e \in[v] \subset \cap_{i=2}^{r+1} \gamma_{i}^{-1}(0)$.

$$
P(x)=\sum_{j=0}^{r}\left(\begin{array}{l}
r \\
j
\end{array}\right) \check{P} x_{1}^{j} e^{r-j}=P\left(x_{1}\right)+\sum_{j=1}^{r-1}\left(\begin{array}{l}
r \\
j
\end{array}\right) \check{P} x_{1}^{j} e^{r-j}+P(e) \text {. Como } P\left(x_{1}\right) \text { e } P(e) \in S
$$

e $S$ é subespaço, basta mostrar que para cada $j \in\{1, \ldots, r-1\}$ fixado, $\check{P} x_{1}^{j} e^{r-j}$ está em $S$.

Para tal consideramos o polinômio $P_{j} \in \mathcal{P}\left({ }^{j} E ; F\right)$ definido por $P_{j}(y) \doteq \check{P} y^{j} e^{r-j}$.

Mostremos que para cada $i \in\{2, \ldots, r-1\}$ temos que $P_{j}\left(\gamma_{i}^{-1}(0)\right) \subset S$. Fixo então $s \in\{2, \ldots, r-1\}$ e seja $y \in \gamma_{s}^{-1}(0)$. Pela fórmula de polarização (2.14),

$$
\check{P} y^{j} e^{r-j}=\frac{1}{r ! 2^{r}} \sum_{\substack{\epsilon_{i}= \pm 1 \\ 1 \leq i \leq r}} \epsilon_{1} \ldots \epsilon_{r} P\left(\left(\epsilon_{1}+\cdots+\epsilon_{j}\right) y+\left(\epsilon_{j+1}+\cdots+\epsilon_{r}\right) e\right) .
$$

Como y e $e \in \gamma_{s}^{-1}(0)$, segue que $\left(\epsilon_{1}+\cdots+\epsilon_{j}\right) y+\left(\epsilon_{j+1}+\cdots+\epsilon_{r}\right) e \in \gamma_{s}^{-1}(0)$ e assim $P\left(\left(\epsilon_{1}+\cdots+\epsilon_{j}\right) y+\left(\epsilon_{j+1}+\cdots+\epsilon_{r}\right) e\right) \in S, \forall \epsilon_{i}= \pm 1,1 \leq i \leq r$.

Portanto, $P_{j}(y)=\check{P} y^{j} e^{r-j} \in S$, como queríamos.

Em particular, $P_{j}\left(\gamma_{i}^{-1}(0)\right) \subset S, \forall i \in\{2, \ldots, j\}$, e assim por hipótese de indução $P_{j}(E) \subset S$, concluindo-se assim a demonstração do lema.

Proposição 3.7. Seja $P \in \mathcal{P}\left({ }^{N} E\right)$ tal que existe $\left\{\gamma_{1}, \ldots, \gamma_{N-1}\right\} \subset E^{\prime}$ linearmente independente com $\gamma_{i}^{-1}(0) \subset C_{P}, \forall i \in\{1, \ldots, N-1\}$. Então $C_{P}=E$.

\section{Demonstração:}

Pelo item (3) da proposição 3.4, basta mostrar que, para cada $j \in\{0, \ldots, N-2\}$, $\phi_{j}(E) \subset \mathcal{P}_{w s c 0}\left({ }^{N-j} E\right)$.

Fixado $j \in\{0, \ldots, N-2\}$, por hipótese $\gamma_{i}^{-1}(0) \subset C_{P}, \forall i \in\{1, \ldots, N-1\}$, e logo $\phi_{j}\left(\gamma_{i}^{-1}(0)\right) \subset \mathcal{P}_{w s c 0}\left({ }^{N-j} E\right), \forall i \in\{1, \ldots, N-1\}$. Como cada $\phi_{j} \in \mathcal{P}\left({ }^{j} E ; \mathcal{P}\left({ }^{N-j} E\right)\right)$ e pelo fato de $\mathcal{P}_{w s c 0}\left({ }^{N-j} E\right)$ ser um subespaço de $\mathcal{P}\left({ }^{N-j} E\right)$, estão satisfeitas as hipóteses do lema com $r=j$, e podemos portanto concluir que $\phi_{j}(E) \subset \mathcal{P}_{w s c 0}\left({ }^{N-j} E\right)$, como queríamos. 
Podemos generalizar a proposição acima da seguinte forma:

Corolário 3.8. Se E é um espaço de Banach tal que $P\left({ }^{i} E\right)=\mathcal{P}_{w s c}\left({ }^{i} E\right), \forall i \in\{1, \ldots, s\}$ e se $P \in \mathcal{P}\left({ }^{N} E\right)$ (com $\left.N>s\right)$ é tal que existe $\left\{\gamma_{1}, \ldots, \gamma_{N-s}\right\} \subset E^{\prime}$ linearmente independente com $\gamma_{i}^{-1}(0) \subset C_{P}, \forall i \in\{1, \ldots, N-s\}$, então $C_{P}=E$.

A demonstração deste fato transcorre diretamente da demonstração da proposição, observando-se que neste caso $\phi_{j}(E) \subset \mathcal{P}_{\text {wsc } 0}\left({ }^{N-j} E\right), \forall j \in\{N-s, \ldots, N-2\}$, pois $P\left({ }^{i} E\right)=$ $\mathcal{P}_{w s c}\left({ }^{i} E\right), \forall i \in\{1, \ldots, s\}$. Para cada $j \in\{0, \ldots, N-s-1\}$, podemos usar o mesmo raciocínio que na demonstração da proposição para provar que $\phi_{j}(E) \subset \mathcal{P}_{w s c 0}\left({ }^{N-j} E\right)$.

A seguir são apresentados alguns exemplos de aplicações da proposição 3.7 .

Exemplo 3.9. Seja $P \in P\left({ }^{5} l_{2}\right)$ definido por $P(x) \doteq x_{1} x_{2} x_{3} \sum_{n=1}^{\infty}\left(x_{n}\right)^{2}$. Então não existe $Q$ homogêneo contínuo de grau $<5 \mathrm{em} \mathrm{l}_{2} \operatorname{com} C_{P}=C_{Q}$.

Primeiro vamos verificar que $C_{P}=\left\{x \in l_{2}: x_{1}=0\right.$ ou $x_{2}=0$ ou $\left.x_{3}=0\right\}$ : seja $x \in\left\{\left(x_{n}\right)_{n} \in l_{2}: x_{1}=0\right.$ ou $x_{2}=0$ ou $\left.x_{3}=0\right\}$ e $\left(x^{k}\right)_{k}$ uma sequência em $l_{2}$ tal que $x^{k} \stackrel{w}{\rightarrow} x$. Mostremos que $\left|P\left(x^{k}\right)-P(x)\right| \rightarrow 0$.

Para cada $k \in \mathbb{N}$,

$$
\left|P\left(x^{k}\right)-P(x)\right|=\left|x_{1}^{k} x_{2}^{k} x_{3}^{k} \sum_{n=1}^{\infty}\left(x_{n}^{k}\right)^{2}\right|=\left|x_{1}^{k}\right|\left|x_{2}^{k}\right|\left|x_{3}^{k}\right|\left|\sum_{n=1}^{\infty}\left(x_{n}^{k}\right)^{2}\right| .
$$

Suponhamos sem perda de generalidade $x_{1}=0$. Pelo corolário 1.34 temos que $x_{1}^{k} \stackrel{k}{\rightarrow}$ $x_{1}=0, x_{2}^{k} \stackrel{k}{\rightarrow} x_{2}$ e $x_{3}^{k} \stackrel{k}{\rightarrow} x_{3}$ e que existe $M \geq 0$ tal que $\left|x_{2}^{k}\right| \leq M,\left|x_{3}^{k}\right| \leq M$ e $\left|\sum_{i=1}^{\infty}\left(x_{i}^{k}\right)^{2}\right| \leq$ $\left\|x^{k}\right\|_{2}^{2} \leq M$, para todo $k$ natural. Então temos que

$$
\left|P\left(x^{k}\right)-P(x)\right|=\left|x_{1}^{k}\right|\left|x_{2}^{k}\right|\left|x_{3}^{k}\right|\left|\sum_{n=1}^{\infty}\left(x_{n}^{k}\right)^{2}\right| \leq M^{3}\left|x_{1}^{k}\right| \rightarrow 0,
$$

como queríamos.

Para mostrarmos a outra inclusão, vamos mostrar que, se $x=\left(x_{n}\right)_{n}$ é tal que $x_{1} \neq 0$, $x_{2} \neq 0$ e $x_{3} \neq 0$, , então $x \notin C_{P}$. Para tal devemos exibir uma sequência $\left(x^{k}\right)_{k}$ convergindo 
fracamente a $x$ tal que $P\left(x^{k}\right) \nrightarrow \rightarrow P(x)$. Seja $\left(x^{k}\right)_{k}$ definida por $x^{k}=\left(x_{1}, \ldots, x_{k-1}, x_{k}+\right.$ $\left.1, x_{k+1}, \ldots\right)=x+e^{k}$. Então $x^{k} \stackrel{w}{\rightarrow} x$ e para $k>3$ temos que

$$
\begin{aligned}
\left|P\left(x^{k}\right)-P(x)\right| & =\left|x_{1}^{k} x_{2}^{k} x_{3}^{k} \sum_{n=1}^{\infty}\left(x_{n}^{k}\right)^{2}-x_{1} x_{2} x_{3} \sum_{n=1}^{\infty}\left(x_{n}\right)^{2}\right| \\
& =\left|x_{1} x_{2} x_{3}\left(\sum_{n \neq k}\left(x_{n}\right)^{2}+\left(x_{k}+1\right)^{2}-\sum_{n=1}^{\infty}\left(x_{n}\right)^{2}\right)\right| \\
& =\left|x_{1}\right|\left|x_{2}\right|\left|x_{3}\right|\left|2 x_{k}+1\right| .
\end{aligned}
$$

Tomando $\epsilon \in] 0,\left|x_{1}\right|\left|x_{2}\right|\left|x_{3}\right|\left[\right.$ e sabendo que $x_{k} \stackrel{k}{\rightarrow} 0$, temos que $\left|P\left(x^{k}\right)-P(x)\right|=$ $\left|x_{1}\right|\left|x_{2}\right|\left|x_{3}\right|\left|2 x_{k}+1\right| \rightarrow\left|x_{1}\right|\left|x_{2}\right|\left|x_{3}\right|>\epsilon$, portanto $P\left(x^{k}\right) \nrightarrow \rightarrow P(x)$, como queríamos.

Suponhamos agora que exista $Q \in \mathcal{P}\left({ }^{r} l_{2}\right), r<5 \operatorname{com} C_{Q}=C_{P}$. Definindo funcionais lineares $\gamma_{1}: x \mapsto x_{1}, \gamma_{2}: x \mapsto x_{2}$ e $\gamma_{3}: x \mapsto x_{3}$, temos que $\gamma_{i}^{-1}(0) \subset C_{P}$ para $i=1,2,3$.

Mas então $\gamma_{i}^{-1}(0) \subset C_{Q}$ para $i=1,2,3$, e pela proposição 3.7 deveríamos ter $C_{Q}=l_{2}$, uma contradição.

Exemplo 3.10. Seja $P \in P\left({ }^{6} l_{3}\right)$ definido por $P(x) \doteq x_{1} x_{2} x_{3} \sum_{n=1}^{\infty}\left(x_{n}\right)^{3}$. Então não existe $Q$ homogêneo contínuo de grau $<6 \mathrm{em} \mathrm{l}_{2} \operatorname{com} C_{P}=C_{Q}$.

Da mesma forma que no exemplo anterior prova-se que $C_{P}=\left\{x \in l_{2}: x_{1}=0\right.$ ou $x_{2}=0$ ou $\left.x_{3}=0\right\}$. Definindo também $\gamma_{1}, \gamma_{2}$ e $\gamma_{3}$ como no exemplo anterior, temos novamente que, se existisse $Q$ r-homogêneo contínuo, $r<6$, com $C_{Q}=C_{P}$, teríamos $\gamma_{i}^{-1}(0) \subset C_{Q}$ para $i=1,2,3$. Lembrando que pelo Teorema de $\operatorname{Pitt}(2.36) \mathcal{P}\left({ }^{2} l_{3}\right)=\mathcal{P}_{w s c}\left({ }^{2} l_{3}\right)$, podemos usar o corolário 3.8 e concluir que $C_{Q}=l_{2}$, novamente contradição.

O Teorema a seguir nos dá uma fórmula para o conjunto de continuidade sequencial fraca para o produto de polinômios homogêneos contínuos, e nos será útil mais adiante, como veremos, inclusive para fazer afirmações sobre irredutibilidade de polinômios homogêneos contínuos.

Lembramos que um polinômio $P \in \mathcal{P}(E)$ é dito redutível se existem polinômios $Q$ e $R$ não constantes tais que $P=Q \cdot R$, onde por definição $Q . R: x \mapsto Q(x) R(x)$, e caso contrário $P$ é dito irredutível. Observe que, se $P$ é homogêneo e se escreve na forma $P=Q \cdot R$, então $Q$ e $R$ são homogêneos. 
Teorema 3.11. Se $P \in \mathcal{P}\left({ }^{N} E\right)$ e $Q \in \mathcal{P}\left({ }^{M} E\right)$, então

$$
C_{P, Q}=\left(C_{P} \cap C_{Q}\right) \cup\left(C_{P} \cap P^{-1}(0)\right) \cup\left(C_{Q} \cap Q^{-1}(0)\right) .
$$

Observe que $P . Q \in \mathcal{P}\left({ }^{N+M} E\right)$. Para demonstrar este Teorema se faz necessário o seguinte lema:

Lema 3.12. Sejam $P \in \mathcal{P}\left({ }^{N} E\right)$ e $x \in E$. Suponhamos que para cada sequência $\left(x_{n}\right)_{n}$ em E com $x_{n} \stackrel{w}{\rightarrow}$ x exista uma subsequência $\left(x_{n_{k}}\right)_{k}$ de $\left(x_{n}\right)_{n} \operatorname{com} P\left(x_{n_{k}}\right) \stackrel{k}{\rightarrow} P(x)$. Então $x \in C_{P}$.

\section{Demonstração (do lema):}

Vamos demonstrar o lema por absurdo. Para tal, dado $x \in E$ tal que $x \notin C_{P}$, precisamos exibir uma sequência $\left(y_{k}\right)_{k}$ convergindo fracamente para $x$ tal que para toda subsequência $\left(y_{k_{j}}\right)_{k}$ de $\left(y_{k}\right)_{k}$ temos que $P\left(y_{k_{j}}\right) \not \rightarrow P(x)$.

Como $x \notin C_{P}$, então existe uma sequência $\left(x_{n}\right)_{n}$ tal que $x_{n} \stackrel{w}{\rightarrow} x$ e $P\left(x_{n}\right) \not \rightarrow P(x)$. Como $P\left(x_{n}\right) \nrightarrow \rightarrow P(x)$, existe $\epsilon>0$ e uma subsequência $\left(x_{n_{k}}\right)_{k}$ de $\left(x_{n}\right)_{n}$ tal que $\mid P\left(x_{n_{k}}\right)-$ $P(x) \mid>\epsilon$, para cada $k \in \mathbb{N}$.

Definindo $\left(y_{k}\right)_{k} \doteq\left(x_{n_{k}}\right)_{k}$, temos que $\left|P\left(y_{k}\right)-P(x)\right|>\epsilon$ para cada $k \in \mathbb{N}$, de forma que para nenhuma subsequência $\left(y_{k_{j}}\right)_{j}$ de $\left(y_{k}\right)_{k}$ podemos ter $P\left(y_{k_{j}}\right) \rightarrow P(x)$. Como além disso $y_{k} \stackrel{w}{\rightarrow} x$, concluimos a demonstração.

\section{Demonstração do Teorema:}

Mostremos primeiro que $C_{P, Q} \supset\left(C_{P} \cap C_{Q}\right) \cup\left(C_{P} \cap P^{-1}(0)\right) \cup\left(C_{Q} \cap Q^{-1}(0)\right)$.

Sejam $x \in\left(C_{P} \cap C_{Q}\right) \cup\left(C_{P} \cap P^{-1}(0)\right) \cup\left(C_{Q} \cap Q^{-1}(0)\right)$ e $\left(x_{n}\right)_{n}$ uma sequência tal que $x_{n} \stackrel{w}{\rightarrow} x$.

Se $x \in C_{P} \cap C_{Q}$, então $P\left(x_{n}\right) \rightarrow P(x)$ e $Q\left(x_{n}\right) \rightarrow Q(x)$, de forma que $(P . Q)\left(x_{n}\right) \rightarrow$ $(P . Q)(x)$. Logo, $x \in C_{P . Q}$.

Se $x \in C_{P} \cap P^{-1}(0)$, então $P\left(x_{n}\right) \rightarrow P(x)=0$. Como $\left\{x_{n}\right\}_{n \in \mathbb{N}}$ é um conjunto limitado e polinômios contínuos levam limitados em limitados, o conjunto $\left\{Q\left(x_{n}\right)\right\}_{n \in \mathbb{N}}$ é limitado em $\mathbb{K}$. Logo, $\lim _{n \rightarrow \infty} P\left(x_{n}\right) Q\left(x_{n}\right)=0=P(x) Q(x)$ e $\operatorname{assim} x \in C_{P . Q}$.

Da mesma forma, se $x \in C_{Q} \cap Q^{-1}(0)$ teremos $x \in C_{P . Q}$.

Para mostrarmos a outra inclusão, considero $x \in C_{P . Q}$ e seja $\left(x_{n}\right)_{n}$ uma sequência tal que $x_{n} \stackrel{w}{\rightarrow} x$. Por hipótese, $P\left(x_{n}\right) Q\left(x_{n}\right) \rightarrow P(x) Q(x)$. 
Como $x_{n} \stackrel{w}{\rightarrow} x$, então para cada $t \in \mathbb{K}$ temos que $x+t\left(x_{n}-x\right) \stackrel{w}{\rightarrow} x$ e consequentemente $P\left(x+t\left(x_{n}-x\right)\right) Q\left(x+t\left(x_{n}-x\right)\right) \rightarrow P(x) Q(x)$.

Pela fórmula binomial (2.13),

$$
\begin{aligned}
P\left(x+t\left(x_{n}-x\right)\right) & =\sum_{j=0}^{N}\left(\begin{array}{c}
N \\
j
\end{array}\right) \check{P} x^{j}\left(t\left(x_{n}-x\right)\right)^{N-j} \\
& =\sum_{j=0}^{N} t^{N-j}\left(\begin{array}{c}
N \\
j
\end{array}\right) \check{P} x^{j}\left(x_{n}-x\right)^{N-j}
\end{aligned}
$$

e

$$
Q\left(x+t\left(x_{n}-x\right)\right)=\sum_{l=0}^{M} t^{M-l}\left(\begin{array}{c}
M \\
l
\end{array}\right) \check{Q} x^{l}\left(x_{n}-x\right)^{M-l} .
$$

Para cada $j \in\{0, \ldots, N\}$, a sequência $\left(\left(\begin{array}{c}N \\ j\end{array}\right) \check{P} x^{j}\left(x_{n}-x\right)^{N-j}\right)_{n \in \mathbb{N}}$ é limitada em $\mathbb{K}$, já que é a imagem de um conjunto limitado por um polinômio homogêneo contínuo. O mesmo vale para as sequências $\left(\left(\begin{array}{c}M \\ l\end{array}\right) \check{Q} x^{l}\left(x_{n}-x\right)^{M-l}\right)_{n \in \mathbb{N}}, \operatorname{com} l \in\{0, \ldots, M\}$. Como toda sequência limitada em $\mathbb{K}$ admite subsequência convergente, e por estarmos trabalhando com uma quantidade finita de sequências limitadas em $\mathbb{K}$, podemos concluir que $\left(x_{n}\right)_{n}$ admite uma subsequência $\left(x_{n_{k}}\right)_{k}$ tal que para cada $j \in\{0, \ldots, N\}$, e cada $l \in\{0, \ldots, M\}$, as sequências

$$
\left(\left(\begin{array}{c}
N \\
j
\end{array}\right) \check{P} x^{j}\left(x_{n_{k}}-x\right)^{N-j}\right)_{k \in \mathbb{N}} \text { e }\left(\left(\begin{array}{c}
M \\
l
\end{array}\right) \check{Q} x^{l}\left(x_{n_{k}}-x\right)^{M-l}\right)_{k \in \mathbb{N}}
$$

convergem.

Para cada $j \in\{0, \ldots, N\}$ e cada $l \in\{0, \ldots, M\}$, definimos

$$
\alpha_{j} \doteq \lim _{k \rightarrow \infty}\left(\begin{array}{c}
N \\
j
\end{array}\right) \check{P} x^{j}\left(x_{n_{k}}-x\right)^{N-j} \text { e } \beta_{l} \doteq \lim _{k \rightarrow \infty}\left(\begin{array}{c}
M \\
l
\end{array}\right) \check{Q} x^{l}\left(x_{n_{k}}-x\right)^{M-l} .
$$

Observe que $\alpha_{N}=P(x)$ e $\beta_{M}=Q(x)$.

Então $P\left(x+t\left(x_{n_{k}}-x\right)\right) \stackrel{k}{\rightarrow} \sum_{j=0}^{N} t^{N-j} \alpha_{j}$ e $Q\left(x+t\left(x_{n_{k}}-x\right)\right) \stackrel{k}{\rightarrow} \sum_{l=0}^{M} t^{M-l} \beta_{l}$ e assim $(P . Q)\left(x+t\left(x_{n_{k}}-x\right)\right) \rightarrow\left(\sum_{j=0}^{N} t^{N-j} \alpha_{j}\right)\left(\sum_{l=0}^{M} t^{M-l} \beta_{l}\right)$. Mas também $(P . Q)\left(x+t\left(x_{n_{k}}-x\right)\right) \rightarrow$ $P(x) Q(x)=\alpha_{N} \beta_{M}$, e assim pela unicidade do limite

$$
\left(\sum_{j=0}^{N} t^{N-j} \alpha_{j}\right)\left(\sum_{l=0}^{M} t^{M-l} \beta_{l}\right)=\alpha_{N} \beta_{M}
$$


Temos que o produto de dois polinômios em $\mathbb{K}$ é igual a uma constante. Portanto ou os dois são constantes, ou um deles é nulo, isto é, pelo menos uma das seguintes afirmações é verdadeira:

(1) $\alpha_{0}=\cdots=\alpha_{N-1}=\beta_{0}=\cdots=\beta_{M-1}=0, \log \lim _{k \rightarrow \infty} P\left(x_{n_{k}}\right)=\lim _{k \rightarrow \infty} P\left(x+\left(x_{n_{k}}-\right.\right.$ $x))=\lim _{k \rightarrow \infty} \sum_{j=0}^{N}\left(\begin{array}{c}N \\ j\end{array}\right) \check{P} x^{j}\left(x_{n_{k}}-x\right)^{N-j}=\sum_{j=0}^{N}\left(\begin{array}{c}N \\ j\end{array}\right) \alpha_{j}=\alpha_{N}=P(x)$ e da mesma forma $\lim _{k \rightarrow \infty} Q\left(x_{n_{k}}\right)=\beta_{M}=Q(x)$

(2) $\alpha_{0}=\cdots=\alpha_{N}=0$, logo $\lim _{k \rightarrow \infty} P\left(x_{n_{k}}\right)=0=P(x)$;

(3) $\beta_{0}=\cdots=\beta_{M}=0$, logo $\lim _{k \rightarrow \infty} Q\left(x_{n_{k}}\right)=0=Q(x)$.

Resumindo, temos a seguinte situação: $x \in C_{P . Q}$ e cada sequência $\left(x_{n}\right)_{n}$ convergindo fracamente a $x$ satisfaz pelo menos uma das seguintes condições:

(1) $\left(x_{n}\right)_{n}$ admite uma subsequência $\left(x_{n_{k}}\right)_{k}$ tal que as sequências dadas por 3.1 convergem, $P\left(x_{n_{k}}\right) \rightarrow P(x)$ e $Q\left(x_{n_{k}}\right) \rightarrow Q(x)$;

(2) $\left(x_{n}\right)_{n}$ admite uma subsequência $\left(x_{n_{k}}\right)_{k}$ tal que as sequências dadas por 3.1 convergem e $P\left(x_{n_{k}}\right) \rightarrow P(x)=0$;

(3) $\left(x_{n}\right)_{n}$ admite uma subsequência $\left(x_{n_{k}}\right)_{k}$ tal que as sequências dadas por 3.1 convergem e $Q\left(x_{n_{k}}\right) \rightarrow Q(x)=0$.

Queremos mostrar que $x \in\left(C_{P} \cap C_{Q}\right) \cup\left(C_{P} \cap P^{-1}(0)\right) \cup\left(C_{Q} \cap Q^{-1}(0)\right)$.

primeiro caso: Suponhamos que $P(x) \neq 0$ e $Q(x) \neq 0$.

Neste caso, cada sequência convergindo fracamente a $x$ não satisfaz (2) nem (3), de forma que satisfaz (1). Então, pelo lema 3.12, $x \in C_{P} \cap C_{Q}$, como queríamos.

segundo caso: Suponhamos que $P(x)=0$ e $Q(x) \neq 0$.

Aqui, cada sequência $\left(x_{n}\right)_{n}$ convergindo fracamente a $x$ não satisfaz (3), e portanto admite uma subsequência $\left(x_{n_{k}}\right)_{k}$ tal que $P\left(x_{n_{k}}\right) \rightarrow P(x)=0$, e de novo pelo mesmo lema concluimos que $x \in C_{P} \cap P^{-1}(0)$.

terceiro caso: $P(x) \neq 0$ e $Q(x)=0$.

Analogamente ao segundo caso, aqui temos que $x \in C_{Q} \cap Q^{-1}(0)$.

quarto caso: $P(x)=Q(x)=0$.

Suponhamos por absurdo que existam duas sequências $\left(x_{n}\right)_{n}$ e $\left(y_{n}\right)_{n}$ convergindo fracamente a $x$ tais que $\left(x_{n}\right)_{n}$ satisfaz (2) mas não satisfaz (3) com a subsequência $\left(x_{n_{k}}\right)_{k}$ e $\left(y_{n}\right)_{n}$ satisfaz (3) e não satisfaz (2) com a subsequência $\left(y_{n_{k}}\right)_{k}$. Vamos definir $\left(u_{k}\right)_{k} \doteq\left(x_{n_{k}}\right)_{k} \mathrm{e}$ $\left(v_{k}\right)_{k} \doteq\left(y_{n_{k}}\right)_{k}$. Então

$$
P\left(u_{k}\right) \rightarrow P(x)=0, Q\left(u_{k}\right) \rightarrow \beta \neq 0 \mathrm{e}
$$


$P\left(v_{k}\right) \rightarrow \alpha \neq 0, Q\left(v_{k}\right) \rightarrow Q(x)=0$.

Como $x \in C_{P . Q}$, temos pela proposição 3.4 que para cada $t \in \mathbb{K},(1+t) x \in C_{P . Q}$. Observe que $(P . Q)((1+t) x)=0$.

Consideremos a sequência $u_{k}+t v_{k}$. temos que $u_{k}+t v_{k} \stackrel{w}{\rightarrow} x+t x=(1+t) x$. Então

$$
(P . Q)\left(u_{k}+t v_{k}\right)=\left(\sum_{j=0}^{N} t^{N-j}\left(\begin{array}{c}
N \\
j
\end{array}\right) \check{P} u_{k}^{j} v_{k}^{N-j}\right)\left(\sum_{l=0}^{M} t^{M-l}\left(\begin{array}{c}
M \\
l
\end{array}\right) \check{Q} u_{k}^{l} v_{k}^{M-l}\right) .
$$

Como as multilineares contínuas levam conjuntos limitados em conjuntos limitados, fica fácil ver que cada $\left(\left(\begin{array}{c}N \\ j\end{array}\right) \check{P} u_{k}^{j} v_{k}^{N-j}\right)_{k}$ e cada $\left(\left(\begin{array}{c}M \\ l\end{array}\right) \check{Q} u_{k}^{l} v_{k}^{M-l}\right)_{k}$ (respectivamente com $j=0, \ldots, N$ e $l=0, \ldots, M)$ é uma sequência limitada em $\mathbb{K}$, e portanto como antes existe uma sequência de índices $\left(k_{m}\right)_{m}$ tal que $\left(u_{k_{m}}\right)_{m}$ e $\left(v_{k_{m}}\right)_{m}$ satisfazem

$$
\begin{aligned}
& \left(\begin{array}{c}
N \\
j
\end{array}\right) \check{P} u_{k_{m}}^{j} v_{k_{m}}^{N-j} \rightarrow a_{j}, \forall j=0, \ldots, N \mathrm{e} \\
& \left(\begin{array}{c}
M \\
l
\end{array}\right) \check{Q} u_{k_{m}}^{l} v_{k_{m}}^{M-l} \rightarrow b_{l}, \forall l=0, \ldots, M .
\end{aligned}
$$

Então

$$
(P . Q)\left(u_{k_{m}}+t v_{k_{m}}\right) \rightarrow\left(\sum_{j=0}^{N} a_{j} t^{N-j}\right)\left(\sum_{l=0}^{M} b_{j} t^{M-l}\right)=0=(P . Q)((1+t) x) .
$$

Temos que o produto de dois polinômios em $\mathbb{K}$ é igual a zero, de onde se conclui que um dos polinômios é nulo, uma contradição com o fato de que $a_{0}=\alpha \neq 0$ e $b_{N}=\beta \neq 0$.

Portanto, se existe uma sequência convergindo fracamente a $x$ e satisfazendo (2), toda sequência convergindo fracamente a $x$ satisfará (2), e podemos concluir pelo lema 3.12 que $x \in C_{P} \cap P^{-1}(0)$. Analogamente, se existe uma sequência convergindo fracamente a $x$ e satisfazendo (3), então $x \in C_{Q} \cap Q^{-1}(0)$.

Aplicando a fórmula do Teorema 3.11 para o caso em que um dos polinômios é um funcional linear, diretamente obtemos o seguinte:

Corolário 3.13. Se $P \in \mathcal{P}\left({ }^{N} E\right)$ e $\gamma \in E^{\prime}$, então $C_{P . \gamma}=C_{P} \cup \gamma^{-1}(0)$

A seguir apresentam-se alguns exemplos de como o Teorema 3.11 pode ser usado para fazer afirmações sobre irredutibilidade de polinômios. Observe que o produto de dois polinômios $P, Q \in \mathcal{P}(E)$, no caso em que $P$ não é homogêneo e $Q$ não é constante, 
não será homogêneo, de forma que para provar que um polinômio homogêneo contínuo é irredutível basta provar que ele não se decompõe em polinômios homogêneos contínuos.

Exemplo 3.14. O polinômio $P \in \mathcal{P}\left({ }^{3} l_{2}\right)$ definido por $P(x) \doteq x_{1} \sum_{n=1}^{\infty}\left(x_{2 n}\right)^{2}+x_{2} \sum_{n=1}^{\infty}\left(x_{2 n+1}\right)^{2}$ é irredutível em $\mathcal{P}(E)$.

Verifica-se que $C_{P}=\left\{x \in l_{2}: x_{1}=x_{2}=0\right\}$ de forma muito similar à que foi feita em exemplos anteriores. Suponha que existam $Q \in \mathcal{P}\left({ }^{2} l_{2}\right)$ e $\gamma \in \mathcal{P}\left({ }^{1} l_{2}\right)=\left(l_{2}\right)^{\prime} \operatorname{com} P=Q \cdot \gamma$. Pelo corolário 3.13, tem-se $C_{P}=C_{Q . \gamma}=C_{Q} \cup \gamma^{-1}(0)$. Mas $\gamma^{-1}(0)$ é hiperplano, e $C_{P}$ não contém hiperplanos, portanto temos uma contradição.

Exemplo 3.15. Considere o espaço $l_{p}$ com $1<p<\infty$ e seja $N=[p]$, onde $[p]$ por definição é o menor inteiro $\geq p$. Então o polinômio $P \in \mathcal{P}\left({ }^{N+1} l_{p}\right)$ definido por $P(x) \doteq$ $x_{1} \sum_{n=1}^{\infty}\left(x_{2 n}\right)^{N}+x_{2} \sum_{n=1}^{\infty}\left(x_{2 n+1}\right)^{N}$ é irredutivel.

Aqui de novo mostra-se que $C_{P}=\left\{x \in l_{p}: x_{1}=x_{2}=0\right\}$ de forma similar a feita anteriormente. Suponha que $P=Q \cdot R$, sendo $Q$ e $R$ polinômios homogêneos contínuos de grau $\geq 1$.

Se $Q$ é linear, então $C_{P}=C_{Q . R}=C_{R} \cup Q^{-1}(0)$, absurdo pois $C_{P}$ não contém hiperplanos. Portanto nem $Q$ nem $R$ podem ter grau 1 .

Se $Q$ tem grau entre 2 e $N-1$, e consequentemente $R$ também, teremos pelo Teorema de Pitt 2.36 que, como os graus de $Q$ e $R$ são menores do que $p, C_{Q}=C_{R}=l_{p}$, e pelo Teorema 3.11 teríamos $C_{P}=l_{p}$, novamente um absurdo. Portanto, $P$ é irredutível.

Exemplo 3.16. O polinômio 3-homogêneo contínuo $Q: L_{p}[0,1] \rightarrow \mathbb{R}, 2<p<\infty$, definido por

$$
Q(f) \doteq \int_{0}^{\frac{1}{2}} f(t) d t \int_{0}^{\frac{1}{2}} f^{2}(t) d t+\int_{\frac{1}{2}}^{1} f(t) d t \int_{\frac{1}{2}}^{1} f^{2}(t) d t
$$

é irredutível.

Vamos estabelecer a seguinte notação para estudar esse exemplo:

$$
Q(f)=\underbrace{\underbrace{\int_{0}^{\frac{1}{2}} f(t) d t}_{\gamma_{1}(f)} \underbrace{\int_{0}^{\frac{1}{2}} f^{2}(t) d t}_{P_{1}(f)}}_{Q_{1}(f)}+\underbrace{\int_{\frac{1}{2}}^{1} f(t) d t}_{Q_{2}(f)} \underbrace{\int_{\frac{1}{2}}^{1} f^{2}(t) d t}_{\gamma_{2}(f)}
$$


Observe que $\gamma_{1}$ e $\gamma_{2}$ são funcionais lineares. Provemos inicialmente que $C_{P_{1}}=\emptyset$. Seja $\left(f_{n}\right)_{n}$ sequência em $L_{p}[0,1]$ definida para cada $n \in \mathbb{N}$ por

$$
f_{n}(t) \doteq \begin{cases}1 & \text { se } t \in\left[0, \frac{1}{2^{2+n}}\left[\cup \left[\frac{4}{2^{2+n}}, \frac{5}{2^{2+n}}\left[\cup \left[\frac{8}{2^{2+n}}, \frac{9}{2^{2+n}}\left[\cup \cdots \cup \left[\frac{2^{n+1}}{2^{2+n}}, \frac{2^{n+1}+1}{2^{2+n}}[;\right.\right.\right.\right.\right.\right.\right. \\ -1 & \text { se } t \in\left[\frac{1}{2^{2+n}}, \frac{2}{2^{2+n}}\left[\cup \left[\frac{5}{2^{2+n}}, \frac{6}{2^{2+n}}\left[\cup \left[\frac{9}{2^{2+n}}, \frac{10}{2^{2+n}}\left[\cup \cdots \cup \left[\frac{2^{n+1}+1}{2^{2+n}}, \frac{2^{n+1}+2}{2^{2+n}}[\right.\right.\right.\right.\right.\right.\right. \\ 0 & \text { caso contrário. }\end{cases}
$$

Observe que, no caso $n=1$, cada união de intervalos na fórmula acima se reduz a um único intervalo. A figura abaixo ilustra os primeiros termos da sequência $\left(f_{n}\right)_{n}$ :

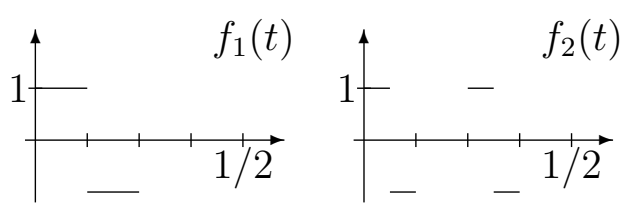

O conjunto $M$ de todos os funcionais lineares da forma

$$
\gamma: f \in L_{p}[0,1] \mapsto \int_{0}^{1} f(t) \chi_{I}(t) d t
$$

onde $I \subset[0,1]$ é um intervalo qualquer e $\chi_{I}$ é a função característica de $I$ satisfaz $\overline{[M]}=$ $\left(L_{p}[0,1]\right)^{\prime}$, e não é difícil ver que para cada $\gamma \in M, \gamma\left(f_{n}\right) \rightarrow 0=\gamma(0)$. Como além disso a sequência $\left(f_{n}\right)_{n}$ é limitada, pela proposição 1.33 podemos concluir que $f_{n} \stackrel{w}{\rightarrow} 0$. Temos também que para cada $n \in \mathbb{N}, P_{1}\left(f_{n}\right)=\frac{1}{4}$, de onde resulta que $0 \notin C_{P_{1}}$ e portanto $C_{P_{1}}=\emptyset$ pelo item (2) proposição 3.4 .

Mostremos agora que $C_{Q}=\left\{f \in L_{p}[0,1]: \int_{0}^{\frac{1}{2}} f(t) d t=\int_{\frac{1}{2}}^{1} f(t) d t=0\right\}=\left\{f \in L_{p}[0,1]\right.$ : $\left.\gamma_{1}(f)=\gamma_{2}(f)=0\right\}$.

Seja $f \in L_{p}[0,1]$ satisfazendo $\gamma_{1}(f)=\gamma_{2}(f)=0$, e seja $\left(g_{n}\right)_{n}$ uma sequência em $L_{p}[0,1]$ convergindo fracamente a $f$. Como $\gamma_{1}$ é um funcional linear, temos que $\gamma_{1}\left(g_{n}\right) \rightarrow \gamma_{1}(f)=$ 0 . Sendo $\left(P_{1}\left(g_{n}\right)\right)_{n}$ limitada, segue que $Q_{1}\left(g_{n}\right)=\gamma_{1}\left(g_{n}\right) P_{1}\left(g_{n}\right) \rightarrow 0$. Da mesma forma, $Q_{2}\left(g_{n}\right) \rightarrow 0$ e assim $Q\left(g_{n}\right) \rightarrow 0=Q(f)$, de onde podemos concluir que $f \in C_{Q}$.

Para mostrar a outra inclusão, seja $f \in C_{Q}$ e verifiquemos que $\gamma_{1}(f)=0$. Consideremos a sequência $\left(f_{n}\right)_{n}$ definida em 3.2. Temos que $f_{n} \stackrel{w}{\rightarrow} 0$ e $f+f_{n} \stackrel{w}{\rightarrow} f$. Para cada $n \in \mathbb{N}$ temos $\gamma_{1}\left(f_{n}\right)=0$, de onde segue que $\gamma_{1}\left(f+f_{n}\right)=\gamma_{1}(f)+\gamma_{1}\left(f_{n}\right)=\gamma_{1}(f)$ e assim $Q_{1}\left(f+f_{n}\right)=\gamma_{1}(f) P_{1}\left(f+f_{n}\right)$. Vale também para cada $n \in \mathbb{N}$ que $Q_{2}\left(f+f_{n}\right)=Q_{2}(f)$ e $\log \mathrm{O}$

$Q\left(f+f_{n}\right)-Q(f)=\gamma_{1}(f) P_{1}\left(f+f_{n}\right)+Q_{2}(f)-\gamma_{1}(f) P_{1}(f)-Q_{2}(f)=\gamma_{1}(f)\left(P_{1}\left(f+f_{n}\right)-P_{1}(f)\right)$. 
Como $f \in C_{Q}$, temos que $Q\left(f+f_{n}\right)-Q(f) \rightarrow 0$. Assim, basta verificar que $P_{1}\left(f+f_{n}\right)-$ $P_{1}(f) \not \rightarrow 0$ para concluir que $\gamma_{1}(f)=0$.

De fato, pela fórmula binomial (2.13)

$$
P_{1}\left(f+f_{n}\right)=P_{1}(f)+2 \int_{0}^{\frac{1}{2}} f(t) f_{n}(t) d t+P_{1}\left(f_{n}\right)
$$

$\int_{0}^{\frac{1}{2}} f(t) f_{n}(t) d t \stackrel{n}{\rightarrow} 0$, pois a aplicação $g \mapsto \int_{0}^{\frac{1}{2}} f(t) g(t) d t$ é linear, e $P_{1}\left(f_{n}\right)=\frac{1}{4}$ para cada $n \in \mathbb{N}$, de forma que $P_{1}\left(f+f_{n}\right)-P_{1}(f) \rightarrow \frac{1}{4} \neq 0$, como queríamos.

Exemplo 3.17. Seja $1<p<\infty, N \doteq[p]$ e $R \in \mathcal{P}\left({ }^{N+2} l_{p}\right)$ definido por

$$
R(x) \doteq \sum_{i=1}^{\infty} \frac{\left(x_{i}\right)^{2}}{2^{i}} \sum_{j \in F_{i}}\left(x_{j}\right)^{N}
$$

onde a sequência $\left(F_{i}\right)_{i}$ forma uma partição de $\mathbb{N}$ em conjuntos infinitos.

Então $R$ é irredutível.

Vamos verificar primeiro que $C_{R}=\{0\}$. Para mostrar que $0 \in C_{R}$, seja $\left(x^{k}\right)_{k}$ uma sequência tal que $x^{k} \stackrel{w}{\rightarrow} 0$, e vamos mostrar que $R\left(x^{k}\right) \rightarrow 0$.

Como $\left(\left\|x^{k}\right\|\right)_{k}$ é limitada, pela proposição $2.34\left(\left\|x^{k}\right\|_{N}\right)_{k}$ e $\left(\left\|x^{k}\right\|_{\infty}\right)_{k}$ também o são, e logo existe $C>0 \mathrm{com}$

$$
\left|\sum_{j \in F_{i}}\left(x_{j}^{k}\right)^{N}\right| \leq C \text { e }\left|x_{i}^{k}\right|^{2} \leq C, \forall i, k \in \mathbb{N}
$$

Supondo $\left(\left\|x^{k}\right\|_{\infty}\right)_{k}$ limitada por $K>0$, temos que $\left|\sum_{i=M+1}^{\infty} \frac{\left(x_{i}^{k}\right)^{2}}{2^{i}}\right| \leq\left|\sum_{i=M+1}^{\infty} \frac{K^{2}}{2^{i}}\right|$ de onde podemos concluir que existe $M_{0} \in \mathbb{N}$ com

$$
\left|\sum_{i=M_{0}+1}^{\infty} \frac{\left(x_{i}^{k}\right)^{2}}{2^{i}}\right| \leq \frac{\epsilon}{2 C}
$$

Como $x_{i}^{k} \stackrel{k}{\rightarrow} 0$, existe $k_{o} \in \mathbb{N}$ com $\left|\sum_{i=1}^{M_{0}} \frac{\left(x_{i}^{k}\right)^{2}}{2^{i}}\right| \leq \frac{\epsilon}{2 C}, \forall k>k_{o}$.

Então $\left|R\left(x^{k}\right)\right| \leq C\left|\sum_{i=1}^{M_{0}} \frac{\left(x_{i}^{k}\right)^{2}}{2^{i}}+\sum_{i=M_{0}+1}^{\infty} \frac{\left(x_{i}^{k}\right)^{2}}{2^{i}}\right| \leq C\left(\frac{\epsilon}{2 C}+\frac{\epsilon}{2 C}\right)=\epsilon, \forall k>k_{o}$, como queríamos. 
Mostremos agora que, dado $x=\left(x_{n}\right)_{n} \in C_{R}$, deve ocorrer $x=0$. Fixemos então $i_{o} \in \mathbb{N}$ e vamos mostrar que $x_{i_{o}}=0$.

Seja $\left(e^{k}\right)_{k}$ a base canônica de $l_{p}$. A sequência $\left(x^{l}\right)_{l \in F_{i_{o}}} \doteq\left(x+e^{l}\right)_{l \in F_{i_{o}}}$ converge fracamente a $x$, portanto $R\left(x^{l}\right) \stackrel{l \in F_{i_{0}}}{\longrightarrow} R(x)$.

Observe que para cada $i \neq i_{o}, \sum_{j \in F_{i}}\left(x_{j}^{l}\right)^{N}=\sum_{j \in F_{i}}\left(x_{j}\right)^{N}$ e que

$$
\sum_{j \in F_{i_{o}}}\left(x_{j}^{l}\right)^{N}=\sum_{\substack{j \in F_{i_{o}} \\
j \neq l}}\left(x_{j}^{l}\right)^{N}+\left(x_{l}+1\right)^{N}=\sum_{j \in F_{i_{o}}}\left(x_{j}\right)^{N}+\sum_{p=0}^{N-1}\left(\begin{array}{c}
N \\
p
\end{array}\right)\left(x_{l}\right)^{p} .
$$

Assim,

$$
\begin{aligned}
R\left(x+e^{l}\right) & =\sum_{i=1}^{\infty} \frac{\left(x_{i}^{l}\right)^{2}}{2^{i}} \sum_{j \in F_{i}}\left(x_{j}\right)^{N}-\frac{\left(x_{i_{o}}^{l}\right)^{2}}{2^{i_{o}}}\left(\sum_{j \in F_{i_{o}}}\left(x_{j}\right)^{N}-\sum_{j \in F_{i_{o}}}\left(x_{j}\right)^{N}-\sum_{p=0}^{N-1}\left(\begin{array}{c}
N \\
p
\end{array}\right)\left(x_{l}\right)^{p}\right) \\
& =\sum_{i=1}^{\infty} \frac{\left(x_{i}^{l}\right)^{2}}{2^{i}} \sum_{j \in F_{i}}\left(x_{j}\right)^{N}+\frac{\left(x_{i_{o}}^{l}\right)^{2}}{2^{i_{o}}} \sum_{p=0}^{N-1}\left(\begin{array}{c}
N \\
p
\end{array}\right)\left(x_{l}\right)^{p} .
\end{aligned}
$$

Vamos mostrar que $\sum_{i=1}^{\infty} \frac{\left(x_{i}^{l}\right)^{2}}{2^{i}} \sum_{j \in F_{i}}\left(x_{j}\right)^{N} \rightarrow R(x)$ : seja $\epsilon>0$. Por um argumento semelhante ao usado para deduzirmos a desigualdade 3.3, existe $C>0$ tal que

$$
\left|\sum_{j \in F_{i}}\left(x_{j}\right)^{N}\right| \leq C, \forall i \in \mathbb{N}
$$

Repetindo agora o raciocínio usado para obter 3.4, existe $M \in \mathbb{N}$ tal que

$$
\left|\sum_{i=M+1}^{\infty} \frac{\left(x_{i}^{l}\right)^{2}}{2^{i}}\right| \leq \frac{\epsilon}{4 C}, \forall l \in F_{i_{o}} \text { e }\left|\sum_{i=M+1}^{\infty} \frac{\left(x_{i}\right)^{2}}{2^{i}}\right| \leq \frac{\epsilon}{4 C}
$$

Existe também $l_{o} \in \mathbb{N}$ tal que, para cada $l \in F_{i_{o}}$ maior do que $l_{o}$,

$$
\left|\sum_{i=1}^{M} \frac{\left(x_{i}^{l}\right)^{2}-\left(x_{i}\right)^{2}}{2^{i}}\right| \leq \frac{\epsilon}{2 C}
$$

pelo fato dessa soma ser finita e por $x_{i}^{l} \stackrel{l \in F_{i o}}{\longrightarrow} x_{i}$.

Então para cada $l \in F_{i_{o}}$ maior do que $l_{o}$,

$$
\begin{aligned}
\left|\sum_{i=1}^{\infty} \frac{\left(x_{i}^{l}\right)^{2}}{2^{i}} \sum_{j \in F_{i}}\left(x_{j}\right)^{N}-R(x)\right| & \leq C\left(\left|\sum_{i=1}^{M} \frac{\left(x_{i}^{l}\right)^{2}-\left(x_{i}\right)^{2}}{2^{i}}\right|+\left|\sum_{i=M+1}^{\infty} \frac{\left(x_{i}^{l}\right)^{2}}{2^{i}}\right|+\left|\sum_{i=M+1}^{\infty} \frac{\left(x_{i}\right)^{2}}{2^{i}}\right|\right) \\
& \leq C\left(\frac{\epsilon}{2 C}+\frac{\epsilon}{4 C}+\frac{\epsilon}{4 C}\right)=\epsilon,
\end{aligned}
$$


como queríamos.

Logo, devemos ter que $\left(\frac{\left(x_{i_{o}}^{l}\right)^{2}}{2_{i_{o}}} \sum_{p=0}^{N-1}\left(\begin{array}{l}N \\ p\end{array}\right)\left(x_{l}\right)^{p}\right)_{l \in F_{i_{o}}}$ não converge a zero. Sabemos que $x_{i_{o}}^{l} \stackrel{l \in F_{i_{o}}}{\longrightarrow} x_{i_{o}}$. Então para mostrar que $x_{i_{o}}=0$ basta mostrar que $\left(\sum_{p=0}^{N-1}\left(\begin{array}{l}N \\ p\end{array}\right)\left(x_{l}\right)^{p}\right)_{l \in F_{i_{o}}}$ não converge a zero. De fato, $\sum_{p=0}^{N-1}\left(\begin{array}{c}N \\ p\end{array}\right)\left(x_{l}\right)^{p} \stackrel{l \in F_{i}}{\longrightarrow} 1$, pois $\left(x_{l}\right)^{p} \stackrel{l \in F_{i_{\rho}}}{\longrightarrow} 0 \forall p=1, \cdots, N-1 \mathrm{e}$ $\left(x_{l}\right)^{0}=1, \forall l \in F_{i_{o}}$.

Completamos assim a demonstração de que $C_{R}=\{0\}$.

Mostremos agora que $R$ é irredutível primeiro no caso em que $E$ é um $\mathbb{C}$-espaço vetorial.

Suponhamos por absurdo que $R$ se decompõe em $P$ e $Q$ não trivialmente. Temos três alternativas:

1) $P$ e $Q$ tem grau $<N$. Neste caso teríamos $C_{P}=C_{Q}=l_{p}$ pelo Teorema de Pitt (2.36) e poderíamos concluir que $C_{R}=l_{p}$ pelo Teorema 3.11, um absurdo;

2) $P \in l_{p}^{\prime}$. Neste caso teríamos pelo corolário 3.13 que $C_{R}=P^{-1}(0) \cup C_{Q}$, absurdo pois $C_{R}$ não contém hiperplanos;

3) $P \in \mathcal{P}\left({ }^{2} l_{p}\right)$. Se $N=2$, pela proposição 3.3 teríamos que $C_{P}$ e $C_{Q}$ são $\emptyset$ ou $l_{p}$ e pelo Teorema 3.11 concluiríamos que $C_{R}$ deve ser, $\emptyset$ ou $l_{p}$, absurdo.

Se $N>2$, suponha primeiro que $C_{P}=\emptyset$. Como $Q$ tem grau $N$, pelo corolário 3.5, $C_{Q}=l_{p}$ ou $\emptyset$, mas não pode ser vazio porque pelo Teorema 3.11 teríamos que $C_{R}=\emptyset$. Então $C_{Q}$ deveria ser $l_{p}$ e pelo Teorema 3.11 teríamos $C_{R}=C_{Q} \cap Q^{-1}(0)=Q^{-1}(0)$.

Se $C_{P} \neq \emptyset$, então novamente pelo corolário $3.5 C_{Q}=l_{p}$ ou $\emptyset$ e pelo Teorema 3.11 deveríamos ter $C_{Q}=\emptyset$. Usando de novo o Teorema 3.11 teríamos que $C_{R} \supset C_{P} \cap P^{-1}(0)=$ $P^{-1}(0)$.

Em ambos os casos acima, teríamos que $C_{R}$ contém imagem inversa da origem por um polinômio homogêneo contínuo de grau $>0$, que pela proposição 2.30 é um conjunto ilimitado, novamente uma contradição.

Provamos portanto que $R$ é irredutível no caso $\mathbb{K}=\mathbb{C}$.

Suponha agora $R$ redutível (em $P, Q$ de forma não trivial) para $\mathbb{K}=\mathbb{R}$. Como pelo corolário 2.39 a complexificação de um polinômio é única, se denotamos por $P^{\prime}, Q^{\prime}$ e $R^{\prime}$ respectivamente os complexificados de $P, Q$ e $R$, deveríamos ter $P^{\prime} \cdot Q^{\prime}=R^{\prime}$, contradição com o que acabamos de provar para $\mathbb{K}=\mathbb{C}$. Portanto, $R$ é irredutível. 
Algums questionamentos naturais com respeito aos conjuntos do tipo $C_{P}$ são analisados ao final desta seção. São eles:

Questão 1: Dado $P \in \mathcal{P}\left({ }^{N} E\right)$, existe $Q$ polinômio homogêneo contínuo de grau menor que $N \operatorname{com} C_{Q}=C_{P}$ ?

Observe inicialmente que, para qualquer espaço de Banach não trivial e qualquer $N \in \mathbb{N}$, existe um polinômio N-homogêneo contínuo $R \operatorname{com} C_{R}=E$ : trivialmente podemos tomar o polinômio nulo, mas também se definirmos $R(x) \doteq(\gamma(x))^{N}$ onde $\gamma$ é um funcional linear não nulo também $C_{R}=E$. Observe que a existência deste polinômio não nulo depende do Teorema de Hahn-Banach (1.1). Desta forma, a resposta é afirmativa quando $C_{P}=E$.

Analisemos então os casos em que $C_{P} \neq E$. Os exemplos 3.9 e 3.10 , mostram que a questão 1 nem sempre tem resposta afirmativa, pelo menos para $N=5$ e 6 . Podemos encontrar exemplos de polinômios homogêneos de qualquer grau maior ou igual a 2 para os quais a questão 1 tem resposta negativa: consideremos os polinômios N-homogêneos contínuos em $l_{p}$, com $1<p<\infty$ e $N \geq p$. Primeiro vamos considerar $N>[p]$. O polinômio $P \in \mathcal{P}\left({ }^{N} l_{p}\right)$ definido por $P(x) \doteq x_{1} \ldots x_{N-[p]} \sum_{n=1}^{\infty}\left(x_{n}\right)^{[p]}$ é tal que $C_{P}=\{x \in$ $l_{p}: x_{1}=0$ ou $\cdots$ ou $\left.x_{N-[p]}=0\right\}$. Usando o Teorema de Pitt (2.36) e o corolário 3.8 em um raciocínio análogo ao feito no exemplo 3.10, mostra-se que não existe polinômio homogêneo contínuo $Q$ em $l_{p}$ de grau menor que $N$ com $C_{Q}=C_{P}$.

Se $N=[p]$, o polinômio $P(x)=\sum_{n=1}^{\infty}\left(x_{n}\right)^{[p]}$ é tal que $C_{P}=\emptyset$ (ver exemplo 2.35). Como pelo Teorema de Pitt (2.36) cada polinômio homogêneo contínuo $Q$ de grau menor que $[p]$ é tal que $C_{Q}=l_{p}$, temos novamente resposta negativa à questão 1 neste caso.

Questão 2: Dado $P \in \mathcal{P}\left({ }^{N} E\right)$, existe $Q \in \mathcal{P}\left({ }^{N+1} E\right)$ com $C_{Q}=C_{P}$ ?

Novamente a resposta é sim, quando $C_{P}=E$. Quando $C_{P}$ contém um hiperplano $H$, existe $\gamma \in E^{\prime} \operatorname{com} \gamma^{-1}(0)=H$ e podemos definir $Q=P . \gamma$. Pelo corolário $3.13, C_{P}=C_{Q}$, e temos portanto que a resposta é afirmativa quando $P$ é tal que $C_{P}$ contém um hiperplano.

Essa questão encontra-se em aberto. Na próxima sessão deste trabalho veremos que sob certas condições podemos ter resposta afirmativa. 
Questão 3: Dados $P, Q \in \mathcal{P}\left({ }^{N} E\right)$, existe $R$ polinômio homogêneo contínuo com $C_{R}=$ $C_{P} \cap C_{Q} ?$

Essa é mais uma questão em aberto, que também será estudada com mais detalhes na próxima sessão.

No caso em que $E$ é um $\mathbb{R}$-espaço de Banach, temos resposta afirmativa, como consequência da proposição a seguir:

Proposição 3.18. Sejam $E$ um $\mathbb{R}$-espaço de Banach, $P \in \mathcal{P}\left({ }^{N} E\right)$ e $Q \in \mathcal{P}\left({ }^{M} E\right)$, com $N \leq M$. Então o polinômio contínuo $R$ definido por $R(x) \doteq P(x)^{2}+Q(x)^{2}$ é tal que $C_{R}=C_{P} \cap C_{Q}$.

Observe que $R$ é de grau $2 M$, e que será homogêneo se e somente se $N=M$.

\section{Demonstração (da proposição):}

Sejam $x \in C_{P} \cap C_{Q}$ e $\left(x_{n}\right)_{n}$ uma sequência tal que $x_{n} \stackrel{w}{\rightarrow} x$. Então $P\left(x_{n}\right) \rightarrow P(x)$ e $Q\left(x_{n}\right) \rightarrow Q(x)$ e assim $P\left(x_{n}\right)^{2}+Q\left(x_{n}\right)^{2} \rightarrow P(x)^{2}+Q(x)^{2}$. Portanto $x \in C_{R}$.

Para mostrar a outra inclusão, como na demonstração do Teorema 3.11 sejam $x \in C_{R}$ e $\left(x_{n}\right)_{n}$ uma sequência tal que $x_{n} \stackrel{w}{\rightarrow} x$ e $t \in \mathbb{R}$. Então para cada $t \in \mathbb{R}$ temos que $x+t\left(x_{n}-x\right) \stackrel{w}{\rightarrow} x$ e pela fórmula binomial (2.13) temos que

$$
\begin{aligned}
& P\left(x+t\left(x_{n}-x\right)\right)=\sum_{j=0}^{N} t^{N-j}\left(\begin{array}{c}
N \\
j
\end{array}\right) \check{P} x^{j}\left(x_{n}-x\right)^{N-j} \mathrm{e} \\
& Q\left(x+t\left(x_{n}-x\right)\right)=\sum_{l=0}^{M} t^{M-l}\left(\begin{array}{c}
M \\
l
\end{array}\right) \check{Q} x^{l}\left(x_{n}-x\right)^{M-l} .
\end{aligned}
$$

Escolhamos, tal como no Teorema citado, uma subsequência $\left(x_{n_{k}}\right)_{k}$ de $\left(x_{n}\right)_{n}$ tal que

$$
\begin{aligned}
& P\left(x+t\left(x_{n_{k}}-x\right)\right) \rightarrow \sum_{j=0}^{N} \alpha_{j} t^{N-j} \mathrm{e} \\
& Q\left(x+t\left(x_{n_{k}}-x\right)\right) \rightarrow \sum_{l=0}^{M} \beta_{j} t^{M-l}, \text { onde } \alpha_{N}=P(x) \text { e } \beta_{M}=Q(x) . \\
& \text { Então } P\left(x+t\left(x_{n_{k}}-x\right)\right)^{2}+Q\left(x+t\left(x_{n_{k}}-x\right)\right)^{2} \rightarrow\left(\sum_{j=0}^{N} \alpha_{j} t^{N-j}\right)^{2}+\left(\sum_{l=0}^{M} \beta_{l} t^{M-l}\right)^{2} .
\end{aligned}
$$

Também temos que $P\left(x+t\left(x_{n}-x\right)\right)^{2}+Q\left(x+t\left(x_{n}-x\right)\right)^{2} \rightarrow P(x)^{2}+Q(x)^{2}=\alpha_{N}^{2}+\beta_{M}^{2}$ e assim pela unicidade do limite

$$
\left(\sum_{j=0}^{N} \alpha_{j} t^{N-j}\right)^{2}+\left(\sum_{l=0}^{M} \beta_{l} t^{M-l}\right)^{2}=\alpha_{N}^{2}+\beta_{M}^{2}
$$


Por se tratarem de polinômios reais, e como temos

$$
0 \leq\left(\sum_{j=0}^{N} \alpha_{j} t^{N-j}\right)^{2}=\alpha_{N}^{2}+\beta_{M}^{2}-\left(\sum_{l=0}^{M} \beta_{l} t^{M-l}\right)^{2} \leq \alpha_{N}^{2}+\beta_{M}^{2}
$$

podemos concluir que $\sum_{j=0}^{N} \alpha_{j} t^{N-j}$ e $\sum_{l=0}^{M} \beta_{l} t^{M-l}$ são polinômios constantes, isto é, que $\alpha_{0}=$ $\cdots=\alpha_{N-1}=\beta_{0}=\cdots=\beta_{M-1}=0$.

Desta forma $P\left(x_{n_{k}}\right)=P\left(x+\left(x_{n_{k}}-x\right)\right)=\sum_{j=0}^{N}\left(\begin{array}{c}N \\ j\end{array}\right) \check{P} x^{j}\left(x_{n_{k}}-x\right)^{N-j} \stackrel{k}{\rightarrow} P(x) \mathrm{e}$ $Q\left(x_{n_{k}}\right) \rightarrow Q(x)$

Portanto pelo lema 3.12 podemos concluir que $x \in C_{P} \cap C_{Q}$.

Usando a mesma técnica de demonstração, temos o seguinte corolário:

Corolário 3.19. Seja $E$ um $\mathbb{R}$-espaço de Banach, e para cada $i \in\{1, \ldots, s\}$ seja $P_{i} \in$ $\mathcal{P}\left({ }^{n_{i}} E\right)$. Então o polinômio contínuo $R$ definido por $R(x) \doteq P_{1}(x)^{2}+\cdots+P_{s}(x)^{2}$ é tal que $C_{R}=C_{P_{1}} \cap \cdots \cap C_{P_{s}}$.

Poderíamos generalizar a proposição 3.18 de forma a responder parcialmente a uma versão mais abrangente da questão 3:

Questão 3': Dados $P_{i} \in \mathcal{P}\left({ }^{N} E\right), i \in \mathbb{N}$, existe polinômio homogêneo contínuo $R$ com $C_{R}=\cap_{i=1}^{\infty} C_{P_{i}}$ ?

Proposição 3.20. Sejam E um $\mathbb{R}$-espaço de Banach e $\left(P_{i}\right)_{i}$ uma sequência em $\mathcal{P}\left({ }^{N} E\right)$. Então o polinômio $R \in \mathcal{P}\left({ }^{2 N} E\right)$ definido por $R(x) \doteq \sum_{i=1}^{\infty} \frac{R_{i}(x)^{2}}{2^{i}}$ (onde $R_{i} \doteq \frac{P_{i}}{\left\|P_{i}\right\|}$ se $P_{i} \neq 0$ e $R_{i} \doteq 0$ se $\left.P_{i}=0\right)$ é tal que $C_{R}=\cap_{i=1}^{\infty} C_{P_{i}}$.

A demonstração dessa proposição depende do seguinte:

Lema 3.21. Sejam $P \in \mathcal{P}\left({ }^{N} E\right)$ e $M>0$. Então $x \in C_{P}$ se e somente se para cada sequência $\left(x_{n}\right)_{n}$ convergindo fracamente para $x$ com $\left\|x_{n}-x\right\| \leq M, \forall n \in \mathbb{N}$ tivermos que $P\left(x_{n}\right) \rightarrow P(x)$.

\section{Demonstração (do lema):}

Uma das implicações é óbvia pela definição do conjunto $C_{P}$. Para a outra consideremos $x$ tal que para toda sequência $\left(x_{n}\right)_{n}$ convergindo fracamente para $x$ satisfazendo $\left\|x_{n}-x\right\| \leq$ $M, \forall n \in \mathbb{N}$, tivermos que $P\left(x_{n}\right) \rightarrow P(x)$, e vamos mostrar que $x \in C_{P}$. 
Seja $\left(y_{n}\right)_{n}$ uma sequência convergindo fracamente a $x$, e seja $B>0$ um limitante para $\left(\left\|y_{n}-x\right\|\right)_{n}$. Observe que $\frac{M}{B} y_{n} \stackrel{w}{\rightarrow} \frac{M}{B} x$ e que $\left\|\frac{M}{B} y_{n}-\frac{M}{B} x\right\|=\frac{M}{B}\left\|y_{n}-x\right\| \leq M$. Então, por hipótese $P\left(\frac{M}{B} y_{n}\right) \rightarrow P\left(\frac{M}{B} x\right)$, portanto $\left(\frac{M}{B}\right)^{N} P\left(y_{n}\right) \rightarrow\left(\frac{M}{B}\right)^{N} P(x)$ e podemos concluir que $P\left(y_{n}\right) \rightarrow P(x)$, como queríamos.

\section{Demonstração (da proposição):}

Seja $Q_{m} \doteq \sum_{i=1}^{m} \frac{R_{i}^{2}}{2^{i}}$. Observe que, como $\left\|R_{i}\right\| \leq 1$, temos que $Q_{m}(x) \rightarrow R(x), \forall x \in E$, e assim podemos concluir pelo Teorema de Banach-Steinhaus para polinômios (2.27) que $R \in \mathcal{P}\left({ }^{2 N} E\right)$.

Mostremos que $C_{R} \supset \cap_{i=1}^{\infty} C_{P_{i}}$. Seja $x \in \cap_{i=1}^{\infty} C_{P_{i}}$. Então $x \in C_{R_{i}}, \forall i \in \mathbb{N}$, e pelo corolário 3.19 temos que $x \in C_{Q_{m}}, \forall m \in \mathbb{N}$. Desta forma, como $Q_{m} \rightarrow R$, pela proposição 2.32 concluimos que $x \in C_{R}$.

Sejam agora $x \in C_{R}$ tal que $\|x\| \leq 1 \mathrm{e}\left(x_{n}\right)_{n}$ uma sequência convergindo fracamente a $x$ satisfazendo $\left\|x_{n}-x\right\| \leq 1$.

Sabemos que para cada $M \in \mathbb{N}$,

$$
\begin{gathered}
\left|R\left(x_{n}\right)-R(x)\right|=\left|\sum_{i=1}^{M} \frac{R_{i}\left(x_{n}\right)^{2}-R_{i}(x)^{2}}{2^{i}}+\sum_{i=M+1}^{\infty} \frac{R_{i}\left(x_{n}\right)^{2}}{2^{i}}-\sum_{i=M+1}^{\infty} \frac{R_{i}(x)^{2}}{2^{i}}\right| \stackrel{n}{\rightarrow} 0 . \\
\text { Observe que }\left|\sum_{i=M+1}^{\infty} \frac{R_{i}(x)^{2}}{2^{i}}\right| \leq \sum_{i=M+1}^{\infty} \frac{1}{2^{i}} \text { e, como }\left\|x_{n}\right\| \leq 2 \text { para cada } n \in \mathbb{N}, \\
|| \sum_{i=M+1}^{\infty} \frac{R_{i}\left(x_{n}\right)^{2}}{2^{i}} \mid \leq \sum_{i=M+1}^{\infty} \frac{1}{2^{i-2}} .
\end{gathered}
$$

Então, dado $\epsilon>0$, existe $M_{0} \in \mathbb{N}$ independente de $x$ e de $\left(x_{n}\right)_{n}$ escolhidos tal que

$$
\left|\sum_{i=M+1}^{\infty} \frac{R_{i}\left(x_{n}\right)^{2}}{2^{i}}-\sum_{i=M+1}^{\infty} \frac{R_{i}(x)^{2}}{2^{i}}\right| \leq \frac{\epsilon}{2}, \text { para cada } M>M_{0} \text { natural. }
$$

Assim, existe $n_{o} \in \mathbb{N}$ com $\left|\sum_{i=1}^{M} \frac{R_{i}\left(x_{n}\right)^{2}-R_{i}(x)^{2}}{2^{i}}\right| \leq \epsilon$, para cada $n>n_{o}$ e cada $M>M_{0}$.

Provamos o seguinte, para o $M_{0}$ encontrado acima: dados $x \in C_{R}$ tal que $\|x\| \leq 1$ e $\left(x_{n}\right)_{n}$ convergindo fracamente a $x$ satisfazendo $\left\|x_{n}-x\right\| \leq 1$, para qualquer $M>M_{0}$ 
temos que $\sum_{i=1}^{M} \frac{R_{i}\left(x_{n}\right)^{2}-R_{i}(x)^{2}}{2^{i}} \stackrel{n}{\rightarrow} 0$, isto é, que $Q_{M}\left(x_{n}\right) \stackrel{n}{\rightarrow} Q_{M}(x)$. Então, pelo lema 3.21, $x \in C_{Q_{M}}$, e pelo corolário 3.19 podemos concluir que $x \in C_{R_{i}}, \forall i \leq M$. Como $M$ é arbitrariamente grande, podemos concluir que $x \in C_{R_{i}}$ para cada $i \in \mathbb{N}$, como queríamos.

Se $x \in C_{R}$ for tal que $\|x\|>1$, então pelo item (2) da proposição $3.4 \frac{x}{\|x\|} \in C_{R}$, e pelo que acabamos de mostrar concluimos que $x \in \cap_{i=1}^{\infty} C_{P_{i}}$.

Questão 4: Dados $P, Q \in \mathcal{P}\left({ }^{N} E\right)$, existe polinômio homogêneo contínuo $R \operatorname{com} C_{R}=$ $C_{P} \cup C_{Q} ?$

Para $R$ de grau $N$ com $N>2$, temos resposta negativa: considero por exemplo os polinômios $P, Q \in \mathcal{P}\left({ }^{N} l_{2}\right)$ definidos por

$$
P(x) \doteq x_{1} \ldots x_{N-2} \sum_{n=1}^{\infty}\left(x_{n}\right)^{2} \text { e } Q(x) \doteq x_{2} \ldots x_{N-1} \sum_{n=1}^{\infty}\left(x_{n}\right)^{2} .
$$

Temos que

$$
\begin{aligned}
& C_{P}=\left\{x \in l_{2} \mid x_{1}=0 \text { ou } \cdots \text { ou } x_{N-2}=0\right\} \mathrm{e} \\
& C_{Q}=\left\{x \in l_{2} \mid x_{2}=0 \text { ou } \cdots \text { ou } x_{N-1}=0\right\} .
\end{aligned}
$$

Por um argumento semelhante ao usado no exemplo 3.9 pode-se concluir a inexistência de $R \in \mathcal{P}\left({ }^{N} l_{2}\right)$ satisfazendo $C_{R}=C_{P} \cup C_{Q}$.

Para $R$ de grau $N=2$ a resposta é afirmativa, já que pelo corolário 3.5 cada polinômio $P \in \mathcal{P}\left({ }^{2} E\right)$ satisfaz $C_{P}=\emptyset$ ou $C_{P}=E$.

Impondo algumas condições adicionais, a questão 4 tem resposta afirmativa, como vemos na proposição a seguir.

Proposição 3.22. Sejam $P, Q \in \mathcal{P}\left({ }^{N} E\right)$. Se existirem $P_{1}, Q_{1} \in \mathcal{P}_{\text {wsc }}\left({ }^{N} E\right)$ tais que $C_{P} \subset\left(P+P_{1}\right)^{-1}(0)$ e $C_{Q} \subset\left(Q+Q_{1}\right)^{-1}(0)$, então $R \doteq\left(P+P_{1}\right) \cdot\left(Q+Q_{1}\right) \in \mathcal{P}\left({ }^{2 N} E\right)$ satisfaz $C_{R}=C_{P} \cup C_{Q}$.

\section{Demonstração:}

Precisaremos utilizar os seguintes dois fatos, considerando $R_{1}$ e $R_{2}$ polinômios quaisquer em $\mathcal{P}\left({ }^{N} E\right)$ :

(1) Se $C_{R_{1}} \subset R_{1}^{-1}(0)$ e $C_{R_{2}} \subset R_{2}^{-1}(0)$, então pelo Teorema 3.11 o polinômio $R_{1} \cdot R_{2} \in$ $\mathcal{P}\left({ }^{2 N} E\right)$ satisfaz $C_{R_{1} . R_{2}}=C_{R_{1}} \cup C_{R_{2}}$, já que

$$
C_{R_{1} . R_{2}}=\left(C_{R_{1}} \cap C_{R_{2}}\right) \cup \underbrace{\left(C_{R_{1}} \cap R_{1}^{-1}(0)\right)}_{C_{R_{1}}} \cup \underbrace{\left(C_{R_{2}} \cap R_{2}^{-1}(0)\right)}_{C_{R_{2}}}=C_{R_{1}} \cup C_{R_{2}} .
$$


(2) Se $R_{2}$ é fracamente sequencialmente contínuo, então $C_{R_{1}+R_{2}}=C_{R_{1}}$ :

Se $x \in C_{R_{1}}$, então para toda sequência $\left(x_{n}\right)_{n}$ convergindo fracamente a $x$ temos que $R_{1}\left(x_{n}\right) \rightarrow R_{1}(x)$. Vale também que $R_{2}\left(x_{n}\right) \rightarrow R_{2}(x)$, de forma que $R_{1}\left(x_{n}\right)+R_{2}\left(x_{n}\right) \rightarrow$ $R_{1}(x)+R_{2}(x)$ e portanto $x \in C_{R_{1}+R_{2}}$.

Se $x \in C_{R_{1}+R_{2}}$, então para toda sequência $\left(x_{n}\right)_{n}$ convergindo fracamente a $x$ temos que $R_{1}\left(x_{n}\right)+R_{2}\left(x_{n}\right) \rightarrow R_{1}(x)+R_{2}(x)$. Logo $R_{1}\left(x_{n}\right)-R_{1}(x)+\underbrace{R_{2}\left(x_{n}\right)-R_{2}(x)}_{\rightarrow 0} \rightarrow 0$, de forma que $R_{1}\left(x_{n}\right)-R_{1}(x) \rightarrow 0$ e podemos concluir que $x \in C_{R_{1}}$.

Seja agora $R$ definida como no enunciado da proposição e vamos mostrar que $C_{R}=$ $C_{P} \cup C_{Q}$. Observe que $C_{P}=C_{P+P_{1}} \subset\left(P+P_{1}\right)^{-1}(0)$ e $C_{Q}=C_{Q+Q_{1}} \subset\left(Q+Q_{1}\right)^{-1}(0)$. Então

$$
C_{R}=C_{\left(P+P_{1}\right) \cdot\left(Q+Q_{1}\right)} \stackrel{(1)}{=} C_{P+P_{1}} \cup C_{Q+Q_{1}} \stackrel{(2)}{=} C_{P} \cup C_{Q},
$$

como queríamos.

Definição 3.23. Dado E, dizemos que um subespaço $S$ de E é complementado em E se existir uma projeção contínua $\Pi$ de $E$ em $S$. Neste caso, П é chamada de projeção associada ao subespaço $S$.

Exemplo 3.24. Sejam $P$ e $Q \in \mathcal{P}\left({ }^{N} E\right)$ tais que $C_{P}$ e $C_{Q}$ são subespaços complementados de $E$, e $\Pi_{P}$ e $\Pi_{Q}$ as projeções associadas a $C_{P}$ e $C_{Q}$, respectivamente. Então o polinômio $R \in \mathcal{P}\left({ }^{2 N} E\right)$ definido por

$$
R \doteq\left(P-P \circ \Pi_{P}\right) \cdot\left(Q-Q \circ \Pi_{Q}\right): x \mapsto\left(P(x)-P\left(\Pi_{P}(x)\right)\right) \cdot\left(Q(x)-Q\left(\Pi_{Q}(x)\right)\right)
$$

satisfaz $C_{R}=C_{P} \cup C_{Q}$.

Basta verificar que o polinômio $R$ satisfaz as condições da proposição 3.22. Vamos verificar que $P \circ \Pi_{P}$ é fracamente sequencialmente contínuo: seja $x \in E$, e seja $\left(x_{n}\right)_{n}$ uma sequência convergindo fracamente a $x$. Pelo fato de $\Pi_{P}: E \rightarrow C_{P}$ ser uma aplicação linear contínua, pela proposição 1.29 temos que $\Pi_{P}\left(x_{n}\right) \stackrel{w}{\rightarrow} \Pi_{P}(x)$ em $E$, e como $\Pi_{P}(x) \in$ $C_{P}$, podemos concluir que $P\left(\Pi_{P}\left(x_{n}\right)\right) \rightarrow P\left(\Pi_{P}(x)\right)$, como queríamos. De forma análoga provamos que $Q \circ \Pi_{Q}$ é fracamente sequencialmente contínuo.

Mostremos agora que $C_{P} \subset\left(P-P \circ \Pi_{P}\right)^{-1}(0)$ : seja $x \in C_{P}$. Então $\Pi_{P}(x)=x$, de forma que $\left(P-P \circ \Pi_{P}\right)(x)=P(x)-P(x)$. Novamente, o raciocínio é análogo para 
provar que $C_{Q} \subset\left(Q-Q \circ \Pi_{Q}\right)^{-1}(0)$.

Observação: Os exemplos 3.1, 3.14 e 3.15 são de polinômios cujos conjuntos de continuidade sequencial fraca são subespaços complementados dos espaços aonde estão definidos. Nem todo subespaço de um espaço de Banach $E$ é complementado. Em [17] se mostra que $c_{o}$ é um subespaço fechado não complementado de $l_{\infty}$.

Da mesma forma que fizemos com a questão 3, podemos também analisar a questão 4 para o caso de uniões infinitas:

Questão 4': Dado para cada $n \in \mathbb{N}$ um polinômio $P_{i} \in \mathcal{P}\left({ }^{N} E\right)$, existe $R$ polinômio homogêneo contínuo com $C_{R}=\cup_{i=1}^{\infty} C_{P_{i}}$ ?

Observe que, pelo item (1) da proposição 3.4, todo polinômio homogêneo contínuo $R$ em $E$ é tal que $C_{R}$ é um conjunto fechado, e que $\cup_{j=1}^{\infty} C_{P_{j}}$ não é necessariamente fechado.

Se definirmos para cada $j \in \mathbb{N}$, por exemplo, $P_{j} \in \mathcal{P}\left({ }^{3} l_{2}\right)$ por

$$
P_{j}(x) \doteq \gamma_{j}(x) \sum_{n=1}^{\infty}\left(x_{n}\right)^{2}, \text { onde } \gamma_{j}(x) \doteq x_{2}-j x_{1},
$$

é facil ver que $C_{P_{j}}=\left\{x \in l_{2} ; x_{2}=j x_{1}\right\}=\gamma_{j}^{-1}(0)$ para cada $j \in \mathbb{N}$. Observamos que $\cup_{j=1}^{\infty} C_{P_{j}}$ não é fechado, e portanto não pode existir um polinômio homogêneo contínuo $R$ de grau algum satisfazendo $C_{R}=\cup_{j=1}^{\infty} C_{P_{j}}$.

Mais do que isso, não existe um polinômio homogêneo contínuo $R$ de grau algum tal que $C_{R}=\overline{\cup_{j=1}^{\infty} C_{P_{j}}}$ : se existisse tal $R$ de grau, digamos, $M$, teríamos que $C_{R} \supset \gamma_{j}^{-1}(0)$ para cada $j \in\{1, \ldots, M-1\}$, e como $\left\{\gamma_{1}, \ldots, \gamma_{M-1}\right\}$ é linearmente independente, pela proposição 3.7 poderíamos concluir que $C_{R}=l_{2}$, uma contradição.

\section{2 $C_{P}$ para espaços de Banach particulares}

Nesta seção serão estudadas propriedades do conjunto $C_{P} \operatorname{com} P \in \mathcal{P}\left({ }^{N} E\right)$, para espaços de Banach $E$ particulares.

No que segue, apresentamos uma caracterização do conjunto $C_{P}$ para espaços de Banach separáveis. Para tal, primeiro será estudado o seguinte resultado: 
Teorema 3.25. Seja $E$ um espaço de Banach separável. Se $P \in \mathcal{P}\left({ }^{N} E\right)$ é tal que $C_{P} \neq \emptyset$, então existe uma sequência $\left(P_{i}\right)_{i}$ de polinômios homogêneos contínuos, cada um de grau entre 1 e $N-2$, tal que

$$
C_{P}=\bigcap_{i=1}^{\infty} P_{i}^{-1}(0) .
$$

Se, adicionalmente, $E$ é tal que $\mathcal{P}_{w s c}\left({ }^{l} E\right)=\mathcal{P}\left({ }^{l} E\right)$ para todo $l \in\{1, \ldots, r-1\}$, então podemos encontrar polinômios homogêneos contínuos de graus entre 1 e $N-r$ satisfazendo a condição acima.

Para demonstrar o Teorema, se faz necessária a seguinte proposição:

Proposição 3.26. Seja E um espaço de Banach separável. Então são verdadeiras as seguintes afirmações:

1) $S e S \subset E$ é um subespaço fechado de $E$, então existe uma sequência $\left(\phi_{i}\right)_{i}$ em $E^{\prime}$ com $S=\bigcap_{i=1}^{\infty} \phi_{i}^{-1}(0)$.

2) Se $S$ é subespaço fechado de um espaço de Banach qualquer $F$ e se $P \in \mathcal{P}\left({ }^{r} E, F\right)$, então existe uma sequência $\left(P_{i}\right)_{i}$ em $\mathcal{P}\left({ }^{r} E\right) \operatorname{com} P^{-1}(S)=\bigcap_{i=1}^{\infty} P_{i}^{-1}(0)$.

\section{Demonstração (da proposição):}

(1) Seja $T$ um complemento algébrico para $S$. Então cada $x \in E$ se escreve de maneira única como $x=y+z$, onde $y \in S$ e $z \in T$.

Como $E$ é separável, existe um conjunto $\left\{z_{i}\right\}_{i \in \mathbb{N}}$ denso em $\{w \in T:\|w\|=1\}$.

Sendo $S$ é fechado, e para cada $i \in \mathbb{N}, z_{i} \notin S$, pelo corolário 1.2 (do Teorema de Hahn-Banach) podemos afirmar que existe, para cada $i \in \mathbb{N}$, um funcional linear contínuo $\phi_{i}$ tal que $\left\|\phi_{i}\right\|=1, \phi_{i}\left(z_{i}\right)=d\left(z_{i}, S\right)$ e $\phi_{i}(S)=\{0\}$. Então $S \subset \phi_{i}^{-1}(0), \forall i \in \mathbb{N}$ e portanto $S \subset \cap_{i=1}^{\infty} \phi_{i}^{-1}(0)$.

Mostremos então que também vale $\cap_{i=1}^{\infty} \phi_{i}^{-1}(0) \subset S$ :

Seja $x \in \cap_{i=1}^{\infty} \phi_{i}^{-1}(0)$, escrito de forma única como $x=y+z$, onde $y \in S$ e $z \in T$. Se mostrarmos que $z=0$, então $x=y \in S$ e assim completaremos a demonstração. Observe que para cada $i \in \mathbb{N}, 0=\phi_{i}(x)=\phi_{i}(y)+\phi_{i}(z)=\phi_{i}(z)$.

Suponha então por absurdo que $z \neq 0$. Neste caso, $\frac{z}{\|z\|} \in\{w \in T:\|w\|=1\}$ e $d\left(\frac{z}{\|z\|}, S\right)>\epsilon$ para algum $\epsilon$ positivo, já que $S$ é fechado. Não é difícil ver que existe $U$ aberto contendo $\frac{z}{\|z\|}$ e tal que $d(u, S)>\epsilon$, para cada $u \in U$. Levando em conta esse fato, e sendo $\left\{z_{i}\right\}_{i \in \mathbb{N}}$ denso em $\{w \in T:\|w\|=1\}$, podemos afirmar que existe uma subsequência $\left(z_{i_{k}}\right)_{k}$ de $\left(z_{i}\right)_{i}$ tal que $z_{i_{k}} \rightarrow \frac{z}{\|z\|}$ e $d\left(z_{i_{k}}, S\right)>\epsilon$ para cada $k$ natural. 
Como $\left|\phi_{i_{k}}\left(\frac{z}{\|z\|}\right)-\phi_{i_{k}}\left(z_{i_{k}}\right)\right|=\left|\phi_{i_{k}}\left(\frac{z}{\|z\|}-z_{i_{k}}\right)\right| \leq\left\|\frac{z}{\|z\|}-z_{i_{k}}\right\| \rightarrow 0$, temos que $\mid \phi_{i_{k}}\left(\frac{z}{\|z\|}\right)-$ $\phi_{i_{k}}\left(z_{i_{k}}\right) \mid \rightarrow 0$. Sendo $\phi_{i_{k}}\left(z_{i_{k}}\right)=d\left(z_{i_{k}}, S\right)>\epsilon$ para cada natural $k$, temos que existe $k_{o} \in \mathbb{N}$ tal que $\left|\phi_{i_{k_{o}}}\left(\frac{z}{\|z\|}\right)\right|>\epsilon$. Uma contradição, pois $\phi_{i_{k_{o}}}\left(\frac{z}{\|z\|}\right)=\frac{1}{\|z\|} \phi_{i_{k_{o}}}(z)=0$.

(2) Pela proposição 1.3 de [20], existem um espaço de Banach $G$ e $\Phi \in \mathcal{P}\left({ }^{r} E ; G\right)$ tais que para cada $P \in \mathcal{P}\left({ }^{r} E ; F\right)$ existe uma única $T \in \mathcal{L}(G ; F) \operatorname{com} T \circ \Phi=P .{ }^{2}$

Como $T$ é contínua e $S$ é fechado, $T^{-1}(S)$ é fechado em $G$, portanto pelo item (1) existe $\left\{\phi_{i}\right\}_{i \in \mathbb{N}} \subset G^{\prime} \operatorname{com} T^{-1}(S)=\cap_{i=1}^{\infty} \phi_{i}^{-1}(0)$.

Considero $P_{i} \doteq \phi_{i} \circ \Phi$. Então $P_{i} \in \mathcal{P}\left({ }^{r} E ; F\right)$ e

$$
\begin{aligned}
\cap_{i=1}^{\infty} P_{i}^{-1}(0) & =\cap_{i=1}^{\infty}\left(\phi_{i} \circ \Phi\right)^{-1}(0) \\
& =\left\{x \in E: \phi_{i}(\Phi(x))=0, \forall i \in \mathbb{N}\right\} \\
& =\left\{x \in E: \Phi(x) \in \cap_{i=1}^{\infty} \phi_{i}^{-1}(0)\right\} \\
& =\left\{x \in E: \Phi(x) \in T^{-1}(S)\right\} \\
& =\{x \in E: T(\Phi(x)) \in S\} \\
& =\{x \in E: P(x) \in S\} \\
& =P^{-1}(S)
\end{aligned}
$$

como queríamos.

\section{Demonstração (do Teorema 3.25):}

Sabemos pela proposição 3.4 e pelo fato de $C_{P} \neq \emptyset$ que $C_{P}=\cap_{j=1}^{N-2}\left\{x \in E: \phi_{j}(x) \in\right.$ $\left.\mathcal{P}_{w s c 0}\left({ }^{N-j} E\right)\right\}=\cap_{j=1}^{N-2} \phi_{j}^{-1}\left(\mathcal{P}_{w s c 0}\left({ }^{N-j} E\right)\right)$. Temos para cada $j \in\{1, \ldots, N-2\}$ que $\phi_{j} \in \mathcal{P}\left(E ; \mathcal{P}\left({ }^{N-j} E\right)\right)$ e $\mathcal{P}_{w s c 0}\left({ }^{N-j} E\right)$ é fechado no espaço de Banach $\mathcal{P}\left({ }^{N-j} E\right)$. Então pelo item (2) da proposição 3.26 podemos concluir que, para cada $j \in\{1, \ldots, N-$ $1\}, \phi_{j}^{-1}\left(\mathcal{P}_{w s c 0}\left({ }^{N-j} E\right)\right)$ é intersecção enumerável de zeros de polinômios j-homogêneos contínuos. Portanto, $C_{P}=\cap_{j=1}^{N-2} \phi_{j}^{-1}\left(\mathcal{P}_{w s c 0}\left({ }^{N-j} E\right)\right)$ é interseç̧ão de zeros de polinômios homogêneos contínuos de graus entre 1 e $N-2$.

O raciocínio é o mesmo para mostrar o resultado no caso em que $E$ é tal que $\mathcal{P}_{w s c}\left({ }^{l} E\right)=$ $\mathcal{P}\left({ }^{l} E\right)$ para cada $l \in\{1, \ldots, r-1\}$ e $\mathcal{P}_{w s c}\left({ }^{r} E\right) \neq \mathcal{P}\left({ }^{r} E\right)$, bastando observar que agora, como $C_{P} \neq \emptyset$, temos que $C_{P}=\cap_{j=1}^{N-r} \phi_{j}^{-1}\left(\mathcal{P}_{w s c 0}\left({ }^{N-j} E\right)\right)$.

\footnotetext{
${ }^{2}$ Este processo é chamado de linearização do polinômio $P$. $G$ na verdade é um subespaço do produto tensorial topológico de $E$, cujo estudo não faz parte do tema desta dissertação.
} 
Estudaremos a seguir o conjunto $C_{P}$ em espaços de Banach com FDD incondicional.

O primeiro resultado a ser apresentado sobre polinômios em espaços de Banach com FDD incondicional, a proposição 3.31, diz respeito a polinômios "diagonais" sob um certo ponto de vista, descritos como na definição abaixo:

Definição 3.27. Seja E espaço um de Banach com FDD incondicional e decomposição $\left\{E_{n}\right\}_{n \in \mathbb{N}}$.

Seja $J=\left(n_{1}, n_{2}, \ldots\right)$ sequência estritamente crescente de naturais. Definimos as projeções associadas à $J \sigma_{j}: E \rightarrow E$ da seguinte maneira:

$$
\sigma_{j}(x) \doteq \begin{cases}\left(\Pi_{n_{j}}-\Pi_{n_{j}-1}\right)(x) & \text { se } j>1 \\ \left(\Pi_{n_{1}}(x)\right. & \text { se } j=1\end{cases}
$$

Definimos os polinômios $\mathbf{N}$-homogêneos diagonais em blocos com relação à $\boldsymbol{J}$ como sendo

$$
\mathcal{D}_{J}\left({ }^{N} E\right) \doteq\left\{P \in \mathcal{P}\left({ }^{N} E\right): P(x)=\sum_{j=1}^{\infty} P\left(\sigma_{j}(x)\right), \forall x \in E\right\} .
$$

Quando $J=\mathbb{N}$, escrevemos simplesmente $\mathcal{D}_{\mathbb{N}}\left({ }^{N} E\right)=\mathcal{D}\left({ }^{N} E\right)$.

Lema 3.28. Sejam $J$ e $J_{1}$ sequências estritamente crescentes de naturais satisfazendo $J_{1} \subset J$. Então $\mathcal{D}_{J}\left({ }^{N} E\right) \subset D_{J_{1}}\left({ }^{N} E\right)$.

\section{Demonstração:}

Sejam $\sigma_{j}$ as projeções associadas à $J=\left(n_{1}, n_{2}, \cdots\right)$ e $\delta_{j}$ as projeções associadas à $J_{1}=\left(m_{1}, m_{2}, \cdots\right)$. Dado $P \in \mathcal{D}_{J}\left({ }^{N} E\right)$, vamos provar que $\sum_{j=1}^{\infty} P\left(\delta_{j}(x)\right)=P(x)$.

Para cada $j \in \mathbb{N}$, existem $k_{1}(j), k_{2}(j) \in \mathbb{N}, k_{1}(j)<k_{2}(j)$, tais que $m_{j-1}=n_{k_{1}(j)}$ e $m_{j}=n_{k_{2}(j)}$. Então $\delta_{j}(x)=\sum_{i=n_{k_{1}(j)}+1}^{n_{k_{2}(j)}} x_{i}=\left(\sigma_{k_{1}(j)+1}+\sigma_{k_{1}(j)+2}+\cdots+\sigma_{k_{2}(j)}\right)(x)$.

Observe que

$$
\begin{aligned}
P\left(\delta_{j}(x)\right) & =P\left(\left(\sigma_{k_{1}(j)+1}+\sigma_{k_{1}(j)+2}+\cdots+\sigma_{k_{2}(j)}\right)(x)\right) \\
& =P\left(\sigma_{k_{1}(j)+1}(x)\right)+P\left(\sigma_{k_{1}(j)+2}(x)\right)+\cdots+P\left(\sigma_{k_{2}(j)}(x)\right),
\end{aligned}
$$

pois $P \in \mathcal{D}_{J}\left({ }^{N} E\right)$, e que $k_{2}(j)=k_{1}(j+1)$. Desta forma,

$$
\begin{aligned}
\sum_{j=1}^{\infty} P\left(\delta_{j}(x)\right) & =\sum_{j=1}^{\infty}\left[P\left(\sigma_{k_{1}(j)+1}(x)\right)+P\left(\sigma_{k_{1}(j)+2}(x)\right)+\cdots+P\left(\sigma_{k_{2}(j)}(x)\right)\right] \\
& =\sum_{i=1}^{\infty} P\left(\sigma_{i}(x)\right)=P(x) .
\end{aligned}
$$


Observe que a igualdade acima é consequência da convergência incondicional da série $\sum_{i=1}^{\infty} P\left(\sigma_{i}(x)\right)$

Exemplo 3.29. Seja $p \in] 1, \infty\left[, N \geq p\right.$ e $P \in \mathcal{P}\left({ }^{N} l_{p}\right)$ definido para cada $\left(x_{n}\right)_{n} \in l_{p}$ por $P(x) \doteq \sum_{n=1}^{\infty} a_{n}\left(x_{n}\right)^{N}$, onde $a=\left(a_{n}\right)_{n} \in l_{\infty}$.

Seja $\bar{J}=\left(n_{1}, n_{2}, \cdots\right) \subset \mathbb{N}$ estritamente crescente. Considero em $l_{p}$ a decomposição $\left\{B_{n}\right\}_{n}$ onde $B_{1} \doteq\left[e^{1}, \cdots, e^{n_{1}}\right]$ e $B_{j} \doteq\left[e^{n_{j-1}+1}, \cdots, e^{n_{j}}\right]$ quando $j \geq 2$. Então $P \in$ $\mathcal{D}_{J}\left({ }^{N} l_{p}\right)$.

Além disso, $C_{P}=l_{p}$ se $a \in c_{o}$, e $C_{P}=\emptyset$ se $a \in l_{\infty} \backslash c_{o}$.

Note que para cada $x=\left(x_{i}\right)_{i} \in l_{p}, \sigma_{j}(x)=(\underbrace{0, \ldots, 0}_{n_{j-1} \text { vezes }}, x_{n_{j-1}+1}, x_{n_{j-1}+2}, \ldots, x_{n_{j}}, 0, \ldots)$. Portanto, $P\left(\sigma_{j}(x)\right)=\sum_{i=n_{j-1}+1}^{n_{j}} a_{i}\left(x_{i}\right)^{N}$ e temos que

$$
\sum_{j=1}^{\infty} P\left(\sigma_{j}(x)\right)=\sum_{i=1}^{\infty} a_{i}\left(x_{i}\right)^{N}=P(x)
$$

e assim verificamos que $P \in \mathcal{D}_{J}\left({ }^{N} l_{p}\right)$.

Verifiquemos agora que se $a \in l_{\infty} \backslash c_{o}$ então $C_{P}=\emptyset$. A base canônica $\left\{e^{k}\right\}_{k}$ é tal que $e^{k} \stackrel{w}{\rightarrow} 0$, mas como $a \notin c_{o}$ temos que $P\left(e^{k}\right)=a_{k} \not \nrightarrow 0$. Isto é, $P$ não é fracamente sequencialmente contínuo na origem, e podemos concluir pelo item (2) da proposição 3.4 que $C_{P}=\emptyset$.

Consideremos agora $a \in c_{o}$. Seja $\left(x^{k}\right)_{k}$ uma sequência em $l_{p}$ tal que $x^{k} \stackrel{w}{\rightarrow} x$,e vamos mostrar que $P\left(x^{k}\right) \rightarrow P(x)$. Usando 2.34 e a limitação da sequência $\left(\left\|x^{k}\right\|\right)_{k}$, temos que existe $B>0$ com $\sum_{i=1}^{\infty}\left|x_{i}^{k}\right|^{N} \leq B$ para cada $k \in \mathbb{N}$ e $\sum_{i=1}^{\infty}\left|x_{i}\right|^{N} \leq B$.

Como $a_{i} \rightarrow 0$, existe $M \in \mathbb{N}$ com $a_{i}<\frac{\epsilon}{4 B}$ para todo $i>M$. Então

$$
\begin{aligned}
\left|P\left(x^{k}\right)-P(x)\right| & =\left|\sum_{i=1}^{\infty} a_{i}\left(x_{i}^{k}\right)^{N}-\sum_{i=1}^{\infty} a_{i}\left(x_{i}\right)^{N}\right| \\
& \leq\left|\sum_{i=1}^{M} a_{i}\left[\left(x_{i}^{k}\right)^{N}-\left(x_{i}\right)^{N}\right]\right|+\left|\sum_{i=M+1}^{\infty} a_{i}\left(x_{i}^{k}\right)^{N}\right|+\left|\sum_{i=M+1}^{\infty} a_{i}\left(x_{i}\right)^{N}\right| .
\end{aligned}
$$

Agora, $\left|\sum_{i=M+1}^{\infty} a_{i}\left(x_{i}^{k}\right)^{N}\right| \leq \frac{\epsilon}{4 B}\left|\sum_{i=M+1}^{\infty}\left(x_{i}^{k}\right)^{N}\right| \leq \frac{\epsilon}{4 B} \sum_{i=1}^{\infty}\left|x_{i}^{k}\right|^{N} \leq \frac{\epsilon}{4}$, para cada $k \in \mathbb{N}$. 
Da mesma forma, $\left|\sum_{i=M+1}^{\infty} a_{i}\left(x_{i}\right)^{N}\right| \leq \frac{\epsilon}{4}$.

Como para cada $i \in \mathbb{N}$ temos que $x_{i}^{k} \stackrel{k}{\rightarrow} x_{i}$, existe $k_{o} \in \mathbb{N}$ tal que $\left|\sum_{i=1}^{M} a_{i}\left[\left(x_{i}^{k}\right)^{N}-\left(x_{i}\right)^{N}\right]\right| \leq$ $\frac{\epsilon}{2}, \forall k>k_{o}$, pelo fato dessa soma ser finita.

Então para cada $k>k_{o},\left|P\left(x^{k}\right)-P(x)\right| \leq \frac{\epsilon}{2}+\frac{\epsilon}{4}+\frac{\epsilon}{4}=\epsilon$, como queríamos.

Exemplo 3.30. Sejam $P \in \mathcal{P}\left({ }^{3} l_{2}\right)$ definido por $P(x) \doteq x_{1} \sum_{i=1}^{\infty}\left(x_{i}\right)^{2}$ e $J=\left(n_{1}, n_{2}, \ldots\right)$ uma sequência crescente de naturais arbitrária. Considero em $l_{2}$ a decomposição $\left\{B_{n}\right\}_{n}$ onde $B_{1} \doteq\left[e^{1}, \cdots, e^{n_{1}}\right]$ e $B_{j} \doteq\left[e^{n_{j-1}+1}, \cdots, e^{n_{j}}\right]$ quando $j \geq 2$. Então $P \notin \mathcal{D}_{J}\left({ }^{3} l_{2}\right)$.

Seja $x \in l_{2}$ com o primeiro e o $n_{2}$-ésimo termos iguais a 1 , e os demais iguais a 0 . Então

$$
\sigma_{j}(x)= \begin{cases}e^{1} & , \text { se } j=1 \\ e^{n_{2}} & , \text { se } j=2 \\ 0 & , \text { caso contrário }\end{cases}
$$

e $\sum_{j=1}^{\infty} P\left(\sigma_{j}(x)\right)=P\left(\sigma_{1}(x)\right)+P\left(\sigma_{2}(x)\right)=1+0=1 \neq P(x)=2$. Portanto $P$ não pode ser diagonal com relação a $J$.

O exemplo 3.29 motiva o seguinte resultado:

Proposição 3.31. Sejam E um espaço de Banach com FDD incondicional e $J$ uma sequência estritamente crescente de naturais. Então

$$
P \in \mathcal{D}_{J}\left({ }^{N} E\right) \Longrightarrow C_{P}=\emptyset \text { ou } E \text {. }
$$

\section{Demonstração:}

Basta mostrar que $x \notin C_{P} \Rightarrow 0 \notin C_{P}$.

Seja $x \in E$ tal que $x \notin C_{P}$. Existe então uma sequência $\left(y_{n}\right)_{n}$ em $E$ satisfazendo $y_{n} \stackrel{w}{\rightarrow} x$ e $\epsilon>0$ tais que $\left|P\left(y_{n}\right)-P(x)\right|>\epsilon, \forall n \in \mathbb{N}$ (tal sequência é produzida da mesma forma que na demonstração do lema 3.12).

Seja $x_{n} \doteq y_{n}-x\left(\log x_{n} \stackrel{w}{\rightarrow} 0\right)$. Vamos mostrar que existe uma sequência de blocos $\left(u_{j}\right)_{j}$ relativa a um certo $J_{1} \subset J$ e uma subsequência $\left(x_{n_{j}}\right)_{j}$ de $\left(x_{n}\right)_{n}$ tal que $\left\|u_{j}-x_{n_{j}}\right\| \rightarrow 0$ e $u_{j} \stackrel{w}{\rightarrow} 0$. 
Para cada $l \in \mathbb{N}$, pelo fato de $\Pi_{l}$ ser uma transformação linear contínua temos pelo corolário 1.29 que $\left(\Pi_{l}\left(x_{n}\right)\right)_{n}$ converge fracamente a zero. Como $\Pi_{l}(E)=\{y \in E: y=$ $\left.y_{1}+\cdots+y_{l}, y_{1} \in E_{1}, \ldots, y_{l} \in E_{l}\right\}$ é isomorfo a $E_{1}+\cdots+E_{l},\left(\Pi_{l}\left(x_{n}\right)\right)_{n}$ pode ser considerada uma sequência convergindo fracamente a zero em $E_{1}+\cdots+E_{l}$, e como esse espaço tem dimensão finita temos que $\Pi_{l}\left(x_{n}\right) \stackrel{n}{\rightarrow} 0$.

Pela definição de $\Pi_{l}$, temos também que para cada $n \in \mathbb{N},\left\|x_{n}-\Pi_{l}\left(x_{n}\right)\right\| \stackrel{l}{\rightarrow} 0$.

Vamos construir a sequência $x_{n_{k}}$ :

$x_{n_{1}} \doteq x_{1}$, e seja $l_{1} \in J$ com $\left\|x_{n_{1}}-\Pi_{l_{1}}\left(x_{n_{1}}\right)\right\|<\frac{1}{2^{1}}$.

Sejam $n_{2}>n_{1} \operatorname{com}\left\|\Pi_{l_{1}}\left(x_{n_{2}}\right)\right\|<\frac{1}{2^{3}}$ e $l_{2}>l_{1}, l_{2} \in J$ com $\left\|x_{n_{2}}-\Pi_{l_{2}}\left(x_{n_{2}}\right)\right\|<\frac{1}{2^{3}}$. Então $\left\|x_{n_{2}}-\left(\Pi_{l_{2}}-\Pi_{l_{1}}\right)\left(x_{n_{2}}\right)\right\|<\frac{1}{2^{3}}+\frac{1}{2^{3}}=\frac{1}{2^{2}}$.

Sejam $n_{3}>n_{2} \operatorname{com}\left\|\Pi_{l_{2}}\left(x_{n_{3}}\right)\right\|<\frac{1}{2^{4}}$ e $l_{3}>l_{2}, l_{3} \in J$ com $\left\|x_{n_{3}}-\Pi_{l_{3}}\left(x_{n_{3}}\right)\right\|<\frac{1}{2^{4}}$. Então $\left\|x_{n_{3}}-\left(\Pi_{l_{3}}-\Pi_{l_{2}}\right)\left(x_{n_{3}}\right)\right\|<\frac{1}{2^{4}}+\frac{1}{2^{4}}=\frac{1}{2^{3}}$.

Desta forma, indutivamente obtemos uma subsequência $\left(x_{n_{j}}\right)_{j}$ de $\left(x_{n}\right)_{n}$ e uma sequência de blocos $\left(u_{j}\right)_{j}$ associada a $J_{1} \doteq\left(l_{1}, l_{2}, \ldots\right)$ definida por $u_{j} \doteq\left(\Pi_{l_{j}}-\Pi_{l_{j-1}}\right)\left(x_{n_{j}}\right)$ tal que $\left\|x_{n_{j}}-u_{j}\right\| \rightarrow 0$.

Falta mostrar que $u_{j} \stackrel{w}{\rightarrow} 0$. Para tal seja $\gamma \in E^{\prime}$ qualquer. Temos que $\gamma\left(u_{j}\right)=$ $\gamma\left(u_{j}-x_{n_{j}}\right)+\gamma\left(x_{n_{j}}\right)$. Como $\left(u_{j}-x_{n_{j}}\right)_{j}$ converge a zero e $\left(\gamma\left(x_{n_{j}}\right)\right)_{j}$ converge a zero pois $x_{n_{j}} \stackrel{w}{\rightarrow} 0$, podemos concluir que $\gamma\left(u_{j}\right) \rightarrow 0$, como queríamos.

Então $\left(u_{j}\right)$ é tal que $\left\|y_{n_{j}}-\left(u_{j}+x\right)\right\| \rightarrow 0$ e $u_{j} \stackrel{w}{\rightarrow} 0$. Como $P$ é uniformemente contínuo sobre os limitados, existe $M>0$ com $\left|P\left(y_{n_{j}}\right)-P\left(u_{j}+x\right)\right| \leq M\left\|y_{n_{j}}-\left(u_{j}+x\right)\right\|, \forall j \in \mathbb{N}$. Seja $j_{o}$ tal que $\left\|y_{n_{j}}-\left(u_{j}+x\right)\right\|<\frac{\epsilon}{2 M}, \forall j>j_{o}$.

Como $\left|P\left(y_{n_{j}}\right)-P(x)\right| \leq\left|P\left(y_{n_{j}}\right)-P\left(u_{j}+x\right)\right|+\left|P\left(u_{j}+x\right)-P(x)\right|$, então para todo $j>j_{\text {o temos que }}$

$\left|P\left(u_{j}+x\right)-P(x)\right| \geq\left|P\left(y_{n_{j}}\right)-P(x)\right|-\left|P\left(y_{n_{j}}\right)-P\left(u_{j}+x\right)\right|>\epsilon-M\left\|y_{n_{j}}-\left(u_{j}+x\right)\right\|>$ $\epsilon-\frac{\epsilon}{2}=\frac{\epsilon}{2}$.

Para provar que $0 \notin C_{P}$, vamos mostrar que $P\left(u_{j}\right) \nrightarrow \rightarrow 0$.

Como $J_{1} \subset J, P \in \mathcal{D}_{J_{1}}\left({ }^{N} E\right)$. Portanto, denominando por $\sigma_{i}$ as projeções associadas a $J_{1}$, teremos que $P(x)=\sum_{i=1}^{\infty} P\left(\sigma_{i}(x)\right)$.

Observe que $\sigma_{i}\left(u_{j}+x\right)=\sigma_{i}\left(u_{j}\right)+\sigma_{i}(x)$ e que

$$
\sigma_{i}\left(u_{j}\right)=\left(\Pi_{l_{i}}-\Pi_{l_{i}-1}\right)\left(\left(\Pi_{l_{j}}-\Pi_{l_{j}-1}\right)\left(x_{n_{j}}\right)\right)= \begin{cases}0 & \text { se } i \neq j \\ u_{j} & \text { se } \mathrm{i}=\mathrm{j}\end{cases}
$$


Então $\sigma_{i}\left(u_{j}+x\right)= \begin{cases}\sigma_{i}(x) & \text { se } i \neq j \\ u_{j}+\sigma_{j}(x) & \text { se } i=j\end{cases}$

Temos então que

$$
\begin{aligned}
& P\left(u_{j}+x\right)=\sum_{i=1}^{\infty} P\left(\sigma_{i}\left(u_{j}+x\right)\right)=\left(\sum_{i \neq j} P\left(\sigma_{i}(x)\right)\right)+P\left(u_{j}+\sigma_{j}(x)\right) \\
& =\underbrace{\left(\sum_{i=1}^{\infty} P\left(\sigma_{i}(x)\right)\right)}_{P(x)}+P\left(u_{j}+\sigma_{j}(x)\right)-P\left(\sigma_{j}(x)\right) .
\end{aligned}
$$

Então $\left|P\left(u_{j}+x\right)-P(x)\right|=\left|P\left(u_{j}+\sigma_{j}(x)\right)-P\left(\sigma_{j}(x)\right)\right|$ e desta forma

$$
\begin{aligned}
\frac{\epsilon}{2}<\left|P\left(u_{j}+\sigma_{j}(x)\right)-P\left(\sigma_{j}(x)\right)\right| & =\left|\sum_{k=1}^{N}\left(\begin{array}{c}
N \\
k
\end{array}\right) \check{P} \sigma_{j}(x)^{N-k} u_{j}^{k}\right| \\
& =\left|\sum_{k=1}^{N-1}\left(\begin{array}{c}
N \\
k
\end{array}\right) \check{P} \sigma_{j}(x)^{N-k} u_{j}^{k}+P\left(u_{j}\right)\right| .
\end{aligned}
$$

Como $\left\|\sigma_{j}(x)\right\| \rightarrow 0$, então $\left|\check{P} \sigma_{j}(x)^{N-k} u_{j}^{k}\right| \leq\|\check{P}\|\left\|\sigma_{j}(x)\right\|^{N-k} \underbrace{\left\|u_{j}\right\|^{k}}_{\text {limitado }} \stackrel{j}{\rightarrow} 0, \forall k \in$ $\{1, \ldots, N-1\}$. Portanto concluimos que $P\left(u_{j}\right)$ não pode convergir a 0 , como queríamos.

É enunciado a seguir um resultado de [6] sem demonstração, mas que será fundamental para a demonstração dos resultados subsequentes.

Proposição 3.32. (proposição $1.3 \mathrm{em} \mathrm{[6])}$

Sejam E um espaço de Banach com FDD incondicional $\left\{E_{n}\right\}_{n}$ e $P \in \mathcal{P}\left({ }^{N} E\right)$. Então existe uma constante $C>0$ tal que para toda $J$ sequência estritamente crescente de naturais,

$$
\sum_{j=1}^{\infty}\left|P\left(\sigma_{j}(x)\right)\right| \leq C\|x\|^{N}, \forall x \in E
$$

onde $\left\{\sigma_{j}\right\}_{j \in \mathbb{N}}$ são as projeções associadas a $\mathrm{J}$.

Proposição 3.33. Seja E um espaço de Banach com FDD incondicional $\left\{E_{n}\right\}_{n}$. Se existir um polinômio de $\mathcal{P}\left({ }^{N} E\right)$ que não é fracamente sequencialmente contínuo, então existirão uma sequência estritamente crescente de naturais $J$ e um polinômio $P \in \mathcal{D}_{J}\left({ }^{N} E\right)$ que não é fracamente sequencialmente contínuo na origem. 


\section{Demonstração:}

Seja $P \in \mathcal{P}\left({ }^{N} E\right) \backslash \mathcal{P}_{w s c 0}\left({ }^{N} E\right)$, e seja $\left(x_{n}\right)_{n}$ uma sequência em $E$ tal que $x_{n} \stackrel{w}{\rightarrow} 0$ e para cada $n$ natural $\left|P\left(x_{n}\right)\right|>\epsilon>0$. Prova-se de maneira análoga a demonstração da proposição 3.31 que existe $J$ sequência estritamente crescente de naturais, $\left(u_{j}\right)_{j}$ sequência de blocos com relação a $J$ e uma subsequência $\left(x_{n_{j}}\right)_{j}$ de $\left(x_{n}\right)_{n}$ tais que $\left\|u_{j}-x_{n_{j}}\right\| \rightarrow 0$ e $u_{j} \stackrel{w}{\rightarrow} 0$.

Definimos, para cada $x \in E$,

$$
Q(x) \doteq \sum_{j=1}^{\infty} P\left(\sigma_{j}(x)\right)
$$

Pela proposição 3.32 , para cada $x \in E$ a série $\sum_{j=1}^{\infty} P\left(\sigma_{j}(x)\right)$ de fato converge, e como para cada $m \in \mathbb{N}$ temos que $\sum_{n=1}^{m} P\left(\sigma_{j}(\cdot)\right) \in \mathcal{P}\left({ }^{N} E\right)$, pelo Teorema de Banach-Steinhaus para polinômios (2.27) concluimos que $Q \in \mathcal{P}\left({ }^{N} E\right)$. Além disso, como $Q\left(\sigma_{j}(x)\right)=$ $P\left(\sigma_{j}(x)\right)$ para cada $x \in E$ e cada $j \in \mathbb{N}$, vale também para cada $x \in E$ que

$$
\sum_{j=1}^{\infty} Q\left(\sigma_{j}(x)\right)=\sum_{j=1}^{\infty} P\left(\sigma_{j}(x)\right)=Q(x)
$$

e portanto $Q \in \mathcal{D}_{J}\left({ }^{N} E\right)$.

Vamos verificar que $Q\left(u_{j}\right) \not \rightarrow 0$. Pela proposição 2.22 , sendo o conjunto $\left\{u_{j} ; j \in\right.$ $\mathbb{N}\} \cup\left\{x_{n_{j}} ; j \in \mathbb{N}\right\}$ limitado em $E$, temos que existe $M \geq 0$ tal que $\left|P\left(u_{j}\right)-P\left(x_{n_{j}}\right)\right| \leq$ $M\left\|u_{j}-x_{n_{j}}\right\|$ para cada $j \in \mathbb{N}$. Então, como $\left\|u_{j}-x_{n_{j}}\right\| \rightarrow 0$, existe $j_{o} \in \mathbb{N}$ tal que $\left|P\left(u_{j}\right)-P\left(x_{n_{j}}\right)\right| \leq \frac{\epsilon}{2}$ para cada $j \geq j_{o}$ natural. Então para cada $j \geq j_{o}$ natural

$$
\left|Q\left(u_{j}\right)\right|=\left|P\left(u_{j}\right)\right| \geq\left|P\left(x_{n_{j}}\right)\right|-\left|P\left(x_{n_{j}}\right)-P\left(u_{j}\right)\right|>\frac{\epsilon}{2}
$$

e assim concluimos que $Q$ não é fracamente sequencialmente contínuo na origem.

Proposição 3.34. Sejam $E$ um espaço de Banach com $F D D$ incondicional e $N \in \mathbb{N}$. Se existir um polinômio de $\mathcal{P}\left({ }^{N} E\right)$ que não é fracamente sequencialmente contínuo, então existirão $J$ subsequência de $\mathbb{N}$, um polinômio $P \in \mathcal{D}_{J}\left({ }^{N} E\right)$ e uma sequência de blocos $\left(u_{j}\right)_{j}$ com respeito à $J$ satisfazendo $\left\|u_{j}\right\|=1$ e $u_{j} \stackrel{w}{\rightarrow} 0$ tais que, para cada sequência $\left(a_{j}\right)_{j}$ em $\mathbb{K}$, tem-se 


$$
\sum_{j=1}^{\infty} a_{j} u_{j} \in \overline{\left[\left\{u_{j}\right\}_{j \in \mathbb{N}}\right]} \Rightarrow P\left(\sum_{j=1}^{\infty} a_{j} u_{j}\right)=\sum_{j=1}^{\infty} a_{j}^{N}
$$

\section{Demonstração:}

De forma análoga à feita na proposição 3.33, podemos mostrar que existem $J$ uma sequência estritamente crescente de naturais, $\left(v_{j}\right)_{j}$ uma sequência de blocos com relação a $J$ e $Q \in \mathcal{D}_{J}\left({ }^{N} E\right)$ tais que, para algum $\epsilon>0,\left|Q\left(v_{j}\right)\right|>\epsilon$ para cada $j \in \mathbb{N}$.

Como $\epsilon<\left|Q\left(v_{j}\right)\right| \leq\|Q\|\left\|v_{j}\right\|$ para cada $j \in \mathbb{N}$, então $\left\|v_{j}\right\|>\frac{\epsilon}{\|Q\|}$ para cada $j \in \mathbb{N}$. Definindo a sequência de blocos com relação a $J\left(u_{j}\right)_{j} \doteq\left(\frac{v_{j}}{\left\|v_{j}\right\|^{N}}\right)_{j}$, temos que $u_{j} \stackrel{w}{\rightarrow} 0$ e para cada $j \in \mathbb{N}$

$$
\left|Q\left(u_{j}\right)\right|=\frac{1}{\left\|v_{j}\right\|^{N}}\left|Q\left(v_{j}\right)\right|>\frac{1}{\left\|v_{j}\right\|^{N}} \epsilon .
$$

Pelo corolário 1.34 a sequência $\left(\left\|v_{j}\right\|\right)_{j}$ é limitada, e assim existe $\epsilon^{\prime}>0$ tal que para cada $j \in \mathbb{N}$

$$
\|Q\| \geq\left|Q\left(u_{j}\right)\right|>\epsilon^{\prime}
$$

Definimos para cada $x \in E$

$$
P(x) \doteq \sum_{j=1}^{\infty} \frac{Q\left(\sigma_{j}(x)\right)}{Q\left(u_{j}\right)}
$$

Para cada $x \in E$ a série acima converge já que pela proposição 3.32 e por 3.5

$$
\left|\sum_{j=1}^{\infty} \frac{Q\left(\sigma_{j}(x)\right)}{Q\left(u_{j}\right)}\right| \leq \frac{1}{\|Q\|} \sum_{j=1}^{\infty}\left|Q\left(\sigma_{j}(x)\right)\right| \leq \frac{C}{\|Q\|}\|x\|^{N},
$$

para alguma constante $C>0$. Usando um argumento análogo ao da demonstração da proposição 3.33 concluimos que $P \in D_{J}\left({ }^{N} E\right)$.

Seja agora $\left(a_{j}\right)_{j}$ uma sequência em $\mathbb{K}$ satisfazendo $\sum_{j=1}^{\infty} a_{j} u_{j} \in \overline{\left[\left\{u_{j}\right\}_{j \in \mathbb{N}}\right]}$. Então

$$
\begin{aligned}
P\left(\sum_{j=1}^{\infty} a_{j} u_{j}\right) & =\sum_{i=1}^{\infty} \frac{Q\left(\sigma_{i}\left(\sum_{j=1}^{\infty} a_{j} u_{j}\right)\right)}{Q\left(u_{i}\right)}=\sum_{i=1}^{\infty} \frac{Q\left(\sigma_{i}\left(a_{i} u_{i}\right)\right)}{Q\left(u_{i}\right)} \\
& =\sum_{i=1}^{\infty} \frac{Q\left(a_{i} \sigma_{i}\left(u_{i}\right)\right)}{Q\left(u_{i}\right)}=\sum_{i=1}^{\infty} \frac{Q\left(a_{i} u_{i}\right)}{Q\left(u_{i}\right)} \\
& =\sum_{i=1}^{\infty} \frac{a_{i}^{N} Q\left(u_{i}\right)}{Q\left(u_{i}\right)}=\sum_{i=1}^{\infty} a_{i}^{N} \mathbf{\Lambda}
\end{aligned}
$$


Como pela proposição 1.21 os espaços de Banach com FDD incondicional são separáveis, pelo corolário 3.5 posso reescrever o Teorema 3.25 da seguinte maneira, para espaços com FDD incondicional:

Proposição 3.35. Seja E um espaço de Banach com FDD incondicional tal que $\mathcal{P}\left({ }^{j} E\right)=$ $\mathcal{P}_{\text {wsc }}\left({ }^{j} E\right), \forall j \in\{1, \ldots, r-1\}$ e $\mathcal{P}\left({ }^{r} E\right) \neq \mathcal{P}_{\text {wsc }}\left({ }^{r} E\right)$. Então as seguintes afirmações são verdadeiras:

1) Se $P \in P\left({ }^{r} E\right)$, então $C_{P}=\emptyset$ ou $C_{P}=E$;

2) $S e P \in P\left({ }^{N} E\right), N>r$, então $C_{P}=\emptyset$ ou existe uma sequência $\left(P_{i}\right)_{i}$ de polinômios homogêneos, cada um de grau entre 1 e $N-r$, tal que $C_{P}=\bigcap_{i=1}^{\infty} P_{i}^{-1}(0)$.

Poderíamos ter escrito a proposição acima com $E$ sendo separável ao invés de ser um espaço com FDD incondicional, tendo assim um resultado mais geral. Escolhemos o enunciado dessa maneira pelo seguinte: observe que os polinômios $\left\{P_{i}\right\}_{i \in \mathbb{N}}$ do item (2) da proposição acima são todos fracamente sequencialmente contínuos quando $N<2 r$. Sendo $E$ um espaço com FDD incondicional, temos que a próxima proposição é uma espécie de reciproca da proposição 3.35:

Proposição 3.36. Seja $E$ um espaço de Banach com FDD incondicional $\left\{E_{n}\right\}_{n}$ tal que $P\left({ }^{j} E\right)=P_{w s c}\left({ }^{j} E\right), \forall j \in\{1, \ldots, r-1\}$ e $P\left({ }^{r} E\right) \neq P_{w s c}\left({ }^{r} E\right)$. Sejam $N$ natural $>r e$ $\left(P_{i}\right)_{i}$ uma sequência de polinômios homogêneos fracamente sequencialmente contínuos em $E$, tendo cada $P_{i}$ grau $n_{i}$ entre 1 e $N-r$. Então existe um polinômio $P \in \mathcal{P}\left({ }^{N} E\right)$ com $C_{P}=\bigcap_{i=1}^{\infty} P_{i}^{-1}(0)$.

\section{Demonstração:}

Pela proposição 3.34, existem $J$ subsequência de $\mathbb{N}$, um polinômio $\Phi \in \mathcal{D}_{J}\left({ }^{r} E\right)$ e uma sequência de blocos $\left(u_{j}\right)_{j}$ com respeito à $J$ satisfazendo $\left\|u_{j}\right\|=1, \forall j \in \mathbb{N}$ e $u_{j} \stackrel{w}{\rightarrow} 0$ tais que para cada $\left(a_{j}\right)_{j}$ sequência em $\mathbb{K}$ com $\sum_{j=1}^{\infty} a_{j} u_{j} \in \overline{\left[\left\{u_{j}\right\}_{j \in \mathbb{N}}\right]}$ tem-se $\Phi\left(\sum_{j=1}^{\infty} a_{j} u_{j}\right)=\sum_{j=1}^{\infty} a_{j}^{r}$.

Em particular, se $\left(a_{j}\right)_{j}=e^{l}$, teremos que $\sum_{j=1}^{\infty} a_{j} u_{j}=u_{l} \in \overline{\left[\left\{u_{j}\right\}_{j \in \mathbb{N}}\right]}$, e assim $\Phi\left(u_{l}\right)=1$, para cada $l \in \mathbb{N}$.

Como $u_{j} \stackrel{w}{\rightarrow} 0$ e $\Phi\left(u_{j}\right) \rightarrow 1$, temos que $\Phi \notin \mathcal{P}_{w s c 0}\left({ }^{r} E\right)$.

Considero para cada $i \in \mathbb{N}$ o polinômio $n_{i}$-homogêneo contínuo

$$
R_{i} \doteq\left\{\begin{array}{ll}
\frac{P_{i}}{\left\|P_{i}\right\|} & \text { se }\left\|P_{i}\right\|>0 \\
0 & \text { se }\left\|P_{i}\right\|=0
\end{array} .\right.
$$


Note que $R_{i}^{-1}(0)=P_{i}^{-1}(0)$, de forma que $\cap_{i=1}^{\infty} R_{i}^{-1}(0)=\cap_{i=1}^{\infty} P_{i}^{-1}(0)$.

Seja $\left(u_{j}^{*}\right)_{j}$ a sequência em $E^{\prime}$ biortogonal a $\left(u_{j}\right)_{j}$. Lembremos que $\left(u_{j}^{*}\right)_{j}$ é definida de forma que $u_{j}^{*}\left(u_{i}\right)=\delta_{i j}$. Defino

$$
P(x) \doteq \sum_{i=1}^{\infty} \frac{R_{i}(x)}{2^{i}} \sum_{j \in F_{i}} \Phi\left(\sigma_{j}(x)\right) u_{j}^{*}\left(\sigma_{j}(x)\right)^{N-r-n_{i}}
$$

onde $\left\{F_{i}\right\}_{i \in \mathbb{N}}$ é uma partição dos naturais em conjuntos infinitos.

Primeiro vamos mostrar que $P$ está bem definido e pertence a $\mathcal{P}\left({ }^{N} E\right)$.

Vamos usar o Teorema de Banach-Steinhaus para polinômios homogêneos (2.27) para mostrar que, para cada $i \in \mathbb{N}, \sum_{j \in F_{i}} \Phi\left(\sigma_{j}(\cdot)\right) u_{j}^{*}\left(\sigma_{j}(\cdot)\right)^{N-r-n_{i}} \in \mathcal{P}\left({ }^{N-n_{i}} E\right)$. Observe que $\sum_{j \in F_{i}, j \leq j_{o}} \Phi\left(\sigma_{j}(\cdot)\right) u_{j}^{*}\left(\sigma_{j}(\cdot)\right)^{N-r-n_{i}} \in \mathcal{P}\left({ }^{N-n_{i}} E\right)$, para cada $j_{o} \in F_{i}$, por se tratarem de somas finitas de polinômios $\left(N-n_{i}\right)$-homogêneos contínuos. Basta verificar então que a série converge pontualmente. Fixemos $x \in E$, e consideremos em $E$ a norma $\||\cdot|||$ introduzida na seção 1.1. Para cada $j>1$ natural,

$$
\left\|\sigma_{j}(x)\right\|=\left\|\Pi_{n_{j}}(x)-\Pi_{n_{j+1}-1}(x)\right\| \leq\left\|\Pi_{n_{j}}(x)\right\|+\left\|\Pi_{n_{j+1}-1}(x)\right\| \leq 2 \mid\|x\| \| .
$$

É fácil ver que vale também a desiguldade acima para $j=1$. Então

$$
\left|u_{j}^{*}\left(\sigma_{j}(x)\right)\right| \leq\left\|u_{j}^{*}\right\|\left\|\sigma_{j}(x)\right\| \leq 2|||x| \| \mid \text { e }\left|u_{j}^{*}\left(\sigma_{j}(x)\right)^{N-r-n_{i}}\right| \leq 2^{N-r-n_{i}} \||| x||^{N-r-n_{i}} .
$$

Assim,

$$
\begin{aligned}
\left|\sum_{j \in F_{i}} \Phi\left(\sigma_{j}(x)\right) u_{j}^{*}\left(\sigma_{j}(x)\right)^{N-r-n_{i}}\right| & \leq \sum_{j \in F_{i}}\left|\Phi\left(\sigma_{j}(x)\right)\right|\left|u_{j}^{*}\left(\sigma_{j}(x)\right)^{N-r-n_{i}}\right| \\
& \leq \sum_{j \in \mathbb{N}}\left|\Phi\left(\sigma_{j}(x)\right)\right|\left|u_{j}^{*}\left(\sigma_{j}(x)\right)^{N-r-n_{i}}\right| \\
& \leq 2^{N-r-n_{i}} \||| x||^{N-r-n_{i}} \sum_{j \in \mathbb{N}}\left|\Phi\left(\sigma_{j}(x)\right)\right| \\
& \leq 2^{N-r-n_{i}}\left\|x \left|\left\|\left.\right|^{N-r-n_{i}} C\right\| x \|^{r}\right.\right.
\end{aligned}
$$

sendo esta última desigualdade devida à proposição 3.32. Portanto, para cada $x$ a série em questão converge e podemos concluir que $\sum_{j \in F_{i}} \Phi\left(\sigma_{j}(\cdot)\right) u_{j}^{*}\left(\sigma_{j}(\cdot)\right)^{N-r-n_{i}} \in \mathcal{P}\left({ }^{N-n_{i}} E\right)$.

Vamos usar de novo o Teorema de Banach-Steinhaus para polinômios homogêneos (2.27) para concluir que

$$
\sum_{i=1}^{\infty} \frac{R_{i}(\cdot)}{2^{i}} \sum_{j \in F_{i}} \Phi\left(\sigma_{j}(\cdot)\right) u_{j}^{*}\left(\sigma_{j}(\cdot)\right)^{N-r-n_{i}} \in \mathcal{P}\left({ }^{N} E\right) .
$$


Para cada $n$ natural, $\sum_{i=1}^{n} \frac{R_{i}(\cdot)}{2^{i}} \sum_{j \in F_{i}} \Phi\left(\sigma_{j}(\cdot)\right) u_{j}^{*}\left(\sigma_{j}(\cdot)\right)^{N-r-n_{i}} \in \mathcal{P}\left({ }^{N} E\right)$, e assim novamente basta mostrar que para $x$ fixado a série é convergente.

De fato,

$$
\begin{aligned}
& \mid \sum_{i=1}^{\infty} \frac{R_{i}(x)}{2^{i}} \sum_{j \in F_{i}} \Phi\left(\sigma_{j}(x)\right) u_{j}^{*}\left(\sigma_{j}(x)\right)^{N-r-n_{i}}\left|\leq \sum_{i=1}^{\infty} \frac{\left|R_{i}(x)\right|}{2^{i}} 2^{N-r-n_{i}}\|\| x\left\|\left.\right|^{N-r-n_{i}} C\right\| x \|^{r}\right. \\
& \leq C\|x\|^{r} 2^{N-r} \sup _{n_{i}=1, \ldots, N-r}\left\{\left.\|x\|\right|^{N-r-n_{i}}\right\} \sum_{i=1}^{\infty} \frac{\left\|R_{i}\right\|\|x\|^{n_{i}}}{2^{i}} \\
& \leq C\|x\|^{r+n_{i}} 2^{N-r} \sup _{n_{i}=1, \ldots, N-r}\left\{\||| x\|^{N-r-n_{i}}\right\} \sum_{i=1}^{\infty} \frac{1}{2^{i}} \\
& \leq C\|x\|^{r+n_{i}} 2^{N-r} \sup _{n_{i}=1, \ldots, N-r}\left\{\left.\||| x\|\right|^{N-r-n_{i}}\right\}<\infty
\end{aligned}
$$

portanto, a série converge pontualmente e $P \in \mathcal{P}\left({ }^{N} E\right)$ está bem definido.

Vamos mostrar agora que $C_{P}=\cap_{i=1}^{\infty} R_{i}^{-1}(0)$.

Para mostrar que $C_{P} \supset \cap_{i=1}^{\infty} R_{i}^{-1}(0)$, sejam $y_{o} \in \cap_{i=1}^{\infty} R_{i}^{-1}(0)$ e $\left(y_{n}\right)_{n}$ uma sequência convergindo fracamente para $y_{o}$. Queremos mostrar que $P\left(y_{n}\right) \rightarrow 0=P\left(y_{o}\right)$. Como pelo corolário $1.34\left(y_{n}\right)_{n}$ é limitada, existe $L>0$ com $\left\|y_{n}\right\| \leq L$ e $\left\|y_{o}\right\| \leq L$. Defino $x_{n} \doteq \frac{y_{n}}{L}$ e $x_{o} \doteq \frac{y_{o}}{L}$. Desta forma temos que $x_{o} \in \cap_{i=1}^{\infty} R_{i}^{-1}(0),\left\|x_{n}\right\| \leq 1, \forall n \in \mathbb{N}$ e $x_{n} \stackrel{w}{\rightarrow} x_{o}$. Precisamos provar então que $P\left(x_{n}\right) \rightarrow 0$.

Pela proposição 1.19 as normas $\|\cdot\|$ e ||$|\cdot|||$ são equivalentes, e portanto existe $K>1$ com \|\|$x\|\mid \leq K\| x \|, \forall x \in E$.

Pelo que já vimos,

$$
\begin{aligned}
\sum_{j \in F_{i}}\left|\Phi\left(\sigma_{j}(x)\right)\right|\left|u_{j}^{*}\left(\sigma_{j}(x)\right)^{N-r-n_{i}}\right| & \leq\left. 2^{N-r-n_{i}}\||x|\|\right|^{N-r-n_{i}} C\|x\|^{r} \\
& \leq 2^{N-r} K^{N-r} C\|x\|^{N-n_{i}}
\end{aligned}
$$

e desta forma para cada $i \in \mathbb{N}$ e cada $x \in E \operatorname{com}\|x\| \leq 1$,

$$
\sum_{j \in F_{i}}\left|\Phi\left(\sigma_{j}\left(x_{n}\right)\right)\right|\left|u_{j}^{*}\left(\sigma_{j}\left(x_{n}\right)\right)^{N-r-n_{i}}\right| \leq C^{\prime} \doteq 2^{N-r} K^{N-r} C .
$$

Então, para cada $n, M \in \mathbb{N}$

$$
\left|P\left(x_{n}\right)\right| \leq C^{\prime} \sum_{i=1}^{\infty} \frac{\left|R_{i}\left(x_{n}\right)\right|}{2^{i}}=C^{\prime}\left(\sum_{i=1}^{M} \frac{\left|R_{i}\left(x_{n}\right)\right|}{2^{i}}+\sum_{i=M+1}^{\infty} \frac{\left|R_{i}\left(x_{n}\right)\right|}{2^{i}}\right) .
$$


Como $\left\|R_{i}\right\| \leq 1, \forall i \in \mathbb{N}$, então $\left|R_{i}\left(x_{n}\right)\right| \leq\left\|R_{i}\right\|\left\|x_{n}\right\| \leq 1, \forall i, n \in \mathbb{N}$. Desta forma, existe $M \in \mathbb{N}$ com $\sum_{i=M+1}^{\infty} \frac{\left|R_{i}\left(x_{n}\right)\right|}{2^{i}} \leq \sum_{i=M+1}^{\infty} \frac{1}{2^{i}}<\frac{\epsilon}{2 C^{\prime}}, \forall n \in \mathbb{N}$.

Como cada $R_{i}$ é fracamente sequencialmente contínuo, existe um natural $n_{o}$ tal que $\sum_{i=1}^{M} \frac{\left|R_{i}\left(x_{n}\right)\right|}{2^{i}}<\frac{\epsilon}{2 C^{\prime}}$ sempre que $n \in \mathbb{N}, n \geq n_{o}$, pelo fato dessa soma ser finita.

Desta maneira, para cada $n \geq n_{o}$ natural temos que $\left|P\left(x_{n}\right)\right|<\epsilon$, como queríamos.

Vamos mostrar agora que $C_{P} \subset \cap_{i=1}^{\infty} R_{i}^{-1}(0)$.

Como pela proposição $3.4 x \in C_{P} \Rightarrow \lambda x \in C_{P}, \forall \lambda \in \mathbb{K}$ e $x \in R_{i}^{-1}(0) \Rightarrow \lambda x \in$ $R_{i}^{-1}(0), \forall \lambda \in \mathbb{K}, \forall i \in \mathbb{N}$, basta mostrar que fixados $x \in C_{P}$ com $\|x\| \leq 1$ e $i_{o} \in \mathbb{N}$ temos que $x \in R_{i_{o}}^{-1}(0)$.

Como $x \in C_{P}$ e $\left(x+u_{l}\right)_{l \in F_{i_{o}}} \stackrel{w}{\rightarrow} x$, então $P\left(x+u_{l}\right) \stackrel{l \in F i_{o}}{\longrightarrow} P(x)$.

Observe que, para $i \neq i_{o}$ vale

$$
\sum_{j \in F_{i}} \Phi\left(\sigma_{j}\left(x+u_{l}\right)\right) u_{j}^{*}\left(\sigma_{j}\left(x+u_{l}\right)\right)^{N-r-n_{i}}=\sum_{j \in F_{i}} \Phi\left(\sigma_{j}(x)\right) u_{j}^{*}\left(\sigma_{j}(x)\right)^{N-r-n_{i}}
$$

e que para $i=i_{o}$

$$
\begin{gathered}
\sum_{j \in F_{i}} \Phi\left(\sigma_{j}\left(x+u_{l}\right)\right) u_{j}^{*}\left(\sigma_{j}\left(x+u_{l}\right)\right)^{N-r-n_{i}} \\
=\sum_{\substack{j \in F_{i_{o}} \\
j \neq l}} \Phi\left(\sigma_{j}(x)\right) u_{j}^{*}\left(\sigma_{j}(x)\right)^{N-r-n_{i}}+\Phi\left(\sigma_{l}(x)+u_{l}\right) u_{l}^{*}\left(\sigma_{l}(x)+u_{l}\right)^{N-r-n_{i_{o}}} .
\end{gathered}
$$

Então para cada $l \in F_{i_{o}}$,

$$
\begin{gathered}
\frac{R_{i_{o}}\left(x+u_{l}\right)}{2^{i_{o}}} \sum_{j \in F_{i_{o}}} \Phi\left(\sigma_{j}\left(x+u_{l}\right)\right) u_{j}^{*}\left(\sigma_{j}\left(x+u_{l}\right)\right)^{N-r-n_{i_{o}}} \\
=\left(\frac{R_{i_{o}}\left(x+u_{l}\right)}{2^{i_{o}}} \sum_{j \in F_{i_{o}}} \Phi\left(\sigma_{j}(x)\right) u_{j}^{*}\left(\sigma_{j}(x)\right)^{N-r-n_{i_{o}}}\right) \\
+\frac{R_{i_{o}}\left(x+u_{l}\right)}{2^{i_{o}}}\left(\Phi\left(\sigma_{l}(x)\right) u_{l}^{*}\left(\sigma_{l}(x)\right)^{N-r-n_{i_{o}}}-\Phi\left(\sigma_{l}(x)+u_{l}\right) u_{l}^{*}\left(\sigma_{l}(x)+u_{l}\right)^{N-r-n_{i_{o}}}\right) .
\end{gathered}
$$

Assim, para $l \in F_{i_{o}}$

$$
P\left(x+u_{l}\right)=\sum_{i=1}^{\infty} \frac{R_{i}\left(x+u_{l}\right)}{2^{i}} \sum_{j \in F_{i}} \Phi\left(\sigma_{j}\left(x+u_{l}\right)\right) u_{j}^{*}\left(\sigma_{j}\left(x+u_{l}\right)\right)^{N-r-n_{i}}
$$




$$
\begin{gathered}
=\left(\sum_{i=1}^{\infty} \frac{R_{i}\left(x+u_{l}\right)}{2^{i}} \sum_{j \in F_{i}} \Phi\left(\sigma_{j}(x)\right) u_{j}^{*}\left(\sigma_{j}(x)\right)^{N-r-n_{i}}\right) \\
+\frac{R_{i_{o}}\left(x+u_{l}\right)}{2^{i_{o}}}\left(\Phi\left(\sigma_{l}(x)\right) u_{l}^{*}\left(\sigma_{l}(x)\right)^{N-r-n_{i_{o}}}-\Phi\left(\sigma_{l}(x)+u_{l}\right) u_{l}^{*}\left(\sigma_{l}(x)+u_{l}\right)^{N-r-n_{i_{o}}}\right) . \\
\text { Vamos provar que } \sum_{i=1}^{\infty} \frac{R_{i}\left(x+u_{l}\right)}{2^{i}} \sum_{j \in F_{i}} \Phi\left(\sigma_{j}(x)\right) u_{j}^{*}\left(\sigma_{j}(x)\right)^{N-r-n_{i}} \stackrel{l \in F_{i_{o}}}{\longrightarrow} P(x):
\end{gathered}
$$

Seja $\epsilon>0$. Vimos que, já que $\|x\| \leq 1$, para todo $i \in \mathbb{N}$ vale

$$
\sum_{j \in F_{i}} \Phi\left(\sigma_{j}(x)\right) u_{j}^{*}\left(\sigma_{j}(x)\right)^{N-r-n_{i}} \leq C^{\prime}
$$

Seja $B>1$ um limitante superior para $\left(\left\|x+u_{l}\right\|\right)_{l \in F_{i_{o}}}$. Como $\left\|\frac{R_{i}}{2^{i}}\right\| \leq \frac{1}{2^{i}}$, segue que para cada $M \in \mathbb{N}$ vale

$$
\left\|\sum_{i=M+1}^{\infty} \frac{R_{i}}{2^{i}}\right\| \leq \sum_{i=M+1}^{\infty}\left\|\frac{R_{i}}{2^{i}}\right\| \leq \sum_{i=M+1}^{\infty} \frac{1}{2^{i}}
$$

Desta forma posso considerar $M_{0}$ satisfazendo $\left\|\sum_{i=M_{0}+1}^{\infty} \frac{R_{i}}{2^{i}}\right\|<\frac{\epsilon}{4 C^{\prime} B}$.

Então para cada $l \in F_{i_{o}}$,

$$
\left|\left(\sum_{i=M_{0}+1}^{\infty} \frac{R_{i}}{2^{i}}\right)\left(x+u_{l}\right)\right| \leq \frac{\epsilon}{4 C^{\prime} B}\left\|x+u_{l}\right\| \leq \frac{\epsilon}{4 C^{\prime}}
$$

e

$$
\left|\left(\sum_{i=M_{0}+1}^{\infty} \frac{R_{i}}{2^{i}}\right)(x)\right| \leq \frac{\epsilon}{4 C^{\prime} B}\|x\| \leq \frac{\epsilon}{4 C^{\prime} B} \leq \frac{\epsilon}{4 C^{\prime}}
$$

Assim,

$$
\begin{gathered}
\left|P(x)-\sum_{i=1}^{\infty} \frac{R_{i}\left(x+u_{l}\right)}{2^{i}} \sum_{j \in F_{i}} \Phi\left(\sigma_{j}(x)\right) u_{j}^{*}\left(\sigma_{j}(x)\right)^{N-r-n_{i}}\right| \\
\leq C^{\prime}\left|\sum_{i=1}^{\infty} \frac{R_{i}(x)}{2^{i}}-\sum_{i=1}^{\infty} \frac{R_{i}\left(x+u_{l}\right)}{2^{i}}\right| \\
=C^{\prime}\left|\sum_{i+1}^{M_{0}} \frac{R_{i}(x)-R_{i}\left(x+u_{l}\right)}{2^{i}}+\sum_{i=M_{0}+1}^{\infty} \frac{R_{i}(x)}{2^{i}}-\sum_{i=M_{0}+1}^{\infty} \frac{R_{i}\left(x+u_{l}\right)}{2^{i}}\right| \\
\leq C^{\prime}\left|\sum_{i+1}^{M_{0}} \frac{R_{i}(x)-R_{i}\left(x+u_{l}\right)}{2^{i}}\right|+\frac{\epsilon}{4}+\frac{\epsilon}{4} .
\end{gathered}
$$


Por hipótese, $R_{i}$ é fracamente sequencialmente contínuo para cada $i \in \mathbb{N}$, de forma que $R_{i}\left(x+u_{l}\right) \stackrel{l \in F_{i_{o}}}{\longrightarrow} R_{i}(x), \forall i \in \mathbb{N}$. Então existe $l_{o} \in F_{i_{o}}$ tal que $\forall l>l_{o}, l \in F_{i_{o}}$ temos

$$
\left|\sum_{i+1}^{M_{0}} \frac{R_{i}(x)-R_{i}\left(x+u_{l}\right)}{2^{i}}\right| \leq \frac{\epsilon}{2 C^{\prime}}
$$

pelo fato de tratar-se de uma soma finita. Assim, para cada $l>l_{o}, l \in F_{i_{o}}$ vale

$$
\left|P(x)-\sum_{i=1}^{\infty} \frac{R_{i}\left(x+u_{l}\right)}{2^{i}} \sum_{j \in F_{i}} \Phi\left(\sigma_{j}(x)\right) u_{j}^{*}\left(\sigma_{j}(x)\right)^{N-r-n_{i}}\right| \leq \epsilon
$$

e provamos o que queríamos.

Temos que, para $l \in F_{i_{o}}$

$$
\begin{gathered}
P\left(x+u_{l}\right)=\left(\sum_{i=1}^{\infty} \frac{R_{i}\left(x+u_{l}\right)}{2^{i}} \sum_{j \in F_{i}} \Phi\left(\sigma_{j}(x)\right) u_{j}^{*}\left(\sigma_{j}(x)\right)^{N-r-n_{i}}\right) \\
+\frac{R_{i_{o}}\left(x+u_{l}\right)}{2^{i_{o}}}\left(\Phi\left(\sigma_{l}(x)\right) u_{l}^{*}\left(\sigma_{l}(x)\right)^{N-r-n_{i_{o}}}-\Phi\left(\sigma_{l}(x)+u_{l}\right) u_{l}^{*}\left(\sigma_{l}(x)+u_{l}\right)^{N-r-n_{i_{o}}}\right) .
\end{gathered}
$$

Além disso, $P\left(x+u_{l}\right) \stackrel{l \in F_{i_{o}}}{\longrightarrow} P(x)$ e vimos que

$$
\sum_{i=1}^{\infty} \frac{R_{i}\left(x+u_{l}\right)}{2^{i}} \sum_{j \in F_{i}} \Phi\left(\sigma_{j}(x)\right) u_{j}^{*}\left(\sigma_{j}(x)\right)^{N-r-n_{i}} \stackrel{l \in F_{i}}{\longrightarrow} P(x)
$$

$\log O$

$$
\frac{R_{i_{o}}\left(x+u_{l}\right)}{2^{i_{o}}}\left(\Phi\left(\sigma_{l}(x)\right) u_{j}^{*}\left(\sigma_{l}(x)\right)^{N-r-n_{i_{o}}}-\Phi\left(\sigma_{l}(x)+u_{l}\right) u_{l}^{*}\left(\sigma_{l}(x)+u_{l}\right)^{N-r-n_{i_{o}}}\right) \stackrel{l \in F_{i_{o}}}{\longrightarrow} 0 .
$$

Mostremos que a sequência

$$
\left(\Phi\left(\sigma_{l}(x)\right) u_{l}^{*}\left(\sigma_{l}(x)\right)^{N-r-n_{i_{o}}}-\Phi\left(\sigma_{l}(x)+u_{l}\right) u_{l}^{*}\left(\sigma_{l}(x)+u_{l}\right)^{N-r-n_{i_{o}}}\right)_{l \in F_{i_{o}}}
$$

não converge para zero, e daí poderemos concluir que $R_{i_{o}}\left(x+u_{l}\right) \stackrel{l \in F_{i_{o}}}{\longrightarrow} 0=R_{i_{o}}(x)$.

Definimos

$$
A_{l}: y \in E \mapsto \Phi(y) u_{j}^{*}(y)^{N-r-n_{i_{o}}} \in \mathbb{K}
$$

Queremos mostrar que a sequência $\left(\left|A_{l}\left(\sigma_{l}(x)\right)-A_{l}\left(\sigma_{l}(x)+u_{l}\right)\right|\right)_{l \in F_{i_{o}}}$ não converge a 0 .

Pela fórmula binomial, 


$$
\begin{aligned}
& A_{l}\left(\sigma_{l}(x)+u_{l}\right)=\sum_{k=0}^{N-n_{i_{o}}}\left(\begin{array}{c}
N-n_{i_{o}} \\
k
\end{array}\right) \check{A}_{l} \sigma_{l}(x)^{k} u_{l}^{N-n_{i_{o}}-k} \\
&=A_{l}\left(\sigma_{l}(x)\right)+\sum_{k=1}^{N-n_{i_{o}}-1}\left(\begin{array}{c}
N-n_{i_{o}} \\
k
\end{array}\right) \check{A}_{l} \sigma_{l}(x)^{k} u_{l}^{N-n_{i_{o}}-k}+\underbrace{A_{l}\left(u_{l}\right)}_{1} . \\
& \text { Então }\left|A_{l}\left(\sigma_{l}(x)\right)-A_{l}\left(\sigma_{l}(x)+u_{l}\right)\right|=\left|\sum_{k=1}^{N-n_{i_{o}}-1}\left(\begin{array}{c}
N-n_{i_{o}} \\
k
\end{array}\right) \check{A}_{l} \sigma_{l}(x)^{k} u_{l}^{N-n_{i_{o}}-k}+1\right| .
\end{aligned}
$$

Pela proposição 2.24,

$$
\begin{aligned}
\left\|\check{A}_{l}\right\| & \leq \frac{\left(N-n_{i_{o}}\right)^{N-n_{i_{o}}}}{\left(N-n_{i_{o}}\right) !}\left\|A_{l}\right\| \\
& \leq \frac{\left(N-n_{i_{o}}\right)^{N-n_{i_{o}}}}{\left(N-n_{i_{o}}\right) !}\|\Phi\|\left\|\left(u_{j}^{*}\right)^{N-r-n_{i_{o}}}\right\| \\
& \leq \frac{\left(N-n_{i_{o}}\right)^{N-n_{i_{o}}}}{\left(N-n_{i_{o}}\right) !}\|\Phi\| .
\end{aligned}
$$

Então para cada $k \in\left\{1, \ldots, N-n_{i_{o}}-1\right\}$ temos que

$$
\begin{aligned}
\left|\check{A}_{l} \sigma_{l}(x)^{k} u_{l}^{N-n_{i_{o}}-k}\right| & \leq\left\|\check{A}_{l}\right\|\left\|\sigma_{l}(x)\right\|^{k}\left\|u_{l}\right\|^{N-n_{i_{o}}-k} \\
& \leq \frac{\left(N-n_{i_{o}}\right)^{N-n_{i_{o}}}}{\left(N-n_{i_{o}}\right) !}\|\Phi\|\left\|\sigma_{l}(x)\right\|^{k}\left\|u_{l}\right\|^{N-n_{i_{o}}-k} \stackrel{l \in F_{i_{o}}}{\longrightarrow} 0
\end{aligned}
$$

pois $\left\|\sigma_{l}(x)\right\| \stackrel{l \in F_{i_{o}}}{\longrightarrow} 0$ e $\left(\left\|u_{l}\right\|^{N-n_{i_{o}}-k}\right)_{l \in F_{i_{o}}}$ é limitada.

Portanto, $\left|A_{l}\left(\sigma_{l}(x)\right)-A_{l}\left(\sigma_{l}(x)+u_{l}\right)\right| \stackrel{l \in F_{i_{o}}}{\longrightarrow}|0-1|=1 \neq 0$, e assim concluímos a demonstração.

Observação: Dentro do contexto da proposição 3.36, o polinômio

$$
\sum_{j=1}^{\infty} \Phi\left(\sigma_{j}(\cdot)\right) u_{j}^{*}\left(\sigma_{j}(\cdot)\right)^{N-r}
$$

com $N \geq r$ não é fracamente sequencialmente contínuo em ponto algum. Temos portanto o seguinte corolário, que é ligeiramente diferente da proposição 3.33:

Corolário 3.37. Seja E um espaço de Banach com FDD incondicional tal que $\mathcal{P}\left({ }^{j} E\right)=$ $\mathcal{P}_{\text {wsc }}\left({ }^{j} E\right), \forall j \in\{1, \ldots, r-1\}$ e $\mathcal{P}\left({ }^{r} E\right) \neq \mathcal{P}_{\text {wsc }}\left({ }^{r} E\right)$. Então existe $J$ uma sequência estritamente crescente de naturais tal que para cada $N$ natural $\geq r$ existe um polinômio $P \in \mathcal{D}_{J}\left({ }^{N} E\right) \operatorname{com} C_{P}=\emptyset$. 
Exemplo 3.38. Seja E um espaço de Banach com FDD incondicional e tal que $\mathcal{P}\left({ }^{j} E\right)=$ $\mathcal{P}_{\text {wsc }}\left({ }^{j} E\right), \forall j \in\{1, \ldots, r-1\}$ e $\mathcal{P}\left({ }^{r} E\right) \neq \mathcal{P}_{\text {wsc }}\left({ }^{r} E\right)$, e seja $N>r$. Então para cada subespaço fechado $S$ de $E$ existe $P \in \mathcal{P}\left({ }^{N} E\right)$ com $C_{P}=S$.

Isto se verifica lembrando que pela proposição 3.26 existe $\left\{\phi_{i}\right\}_{i \in \mathbb{N}} \subset E^{\prime}$ com $S=$ $\cap_{i=1}^{\infty} \phi_{i}^{-1}(0)$. Então pela proposição 3.36 existe $P \in \mathcal{P}\left({ }^{N} E\right) \operatorname{com} C_{P}=\cap_{i=1}^{\infty} \phi_{i}^{-1}(0)=S$.

Em particular, seja $E=l_{p}$ e $N>[p]$, e seja $S=\cap_{i=1}^{\infty} \phi_{i}^{-1}(0)$ um subespaço fechado qualquer de $l_{p}$. Então, se $\left\{F_{i}\right\}_{i \in \mathbb{N}}$ é partição dos naturais em conjuntos infinitos, o polinômio N-homogêneo

$$
P(x) \doteq \sum_{i=1}^{\infty} \frac{\phi_{i}(x)}{2^{i}} \sum_{j \in F_{i}} x_{j}^{N-1}
$$

é tal que $C_{P}=S$.

A seguir são apresentados resultados relacionados com as questões colocadas ao final da seção anterior, no caso em que $E$ é espaço de Banach com FDD incondicional. Utilizaremos para nossas construções o polinômio $\Phi$ tal como foi definido no início da demonstração da proposição 3.36 .

Corolário 3.39. Seja E um espaço de Banach com FDD incondicional tal que $\mathcal{P}\left({ }^{j} E\right)=$ $\mathcal{P}_{\text {wsc }}\left({ }^{j} E\right), \forall j \in\{1, \ldots, r-1\}$ e $\mathcal{P}\left({ }^{r} E\right) \neq \mathcal{P}_{\text {wsc }}\left({ }^{r} E\right)$. Suponhamos $N$ natural $>r e$ que $P \in \mathcal{P}\left({ }^{N} E\right)$ é tal que $C_{P}=\emptyset$ ou $C_{P}=\bigcap_{i=1}^{\infty} P_{i}^{-1}(0)$, onde cada $P_{i}$ é fracamente sequencialmente contínuo de grau entre 1 e $N-r$. Então existe $Q \in \mathcal{P}\left({ }^{N+1} E\right)$ com $C_{Q}=C_{P}$

\section{Demonstração:}

No caso em que $C_{P}=\bigcap_{i=1}^{\infty} P_{i}^{-1}(0)$, supondo $P_{i} \neq 0, \forall i \in \mathbb{N}$, note que se definirmos

$$
Q(x) \doteq \sum_{i=1}^{\infty} \frac{P_{i}(x)}{\left\|P_{i}\right\| 2^{i}} \sum_{j \in F_{i}} \Phi\left(\sigma_{j}(x)\right) u_{j}^{*}\left(\sigma_{j}(x)\right)^{N-r-n_{i}+1}
$$

de forma análoga à demonstração da proposição 3.36, teremos $Q \in \mathcal{P}\left({ }^{N+1} E\right)$ e $C_{Q}=C_{P}$.

No caso em que $C_{P}=\emptyset$, o resultado é evidente pelo corolário 3.37 .

O corolário 3.39 nos forneceu solução parcial para a questão 2. O corolário seguinte fornece solução parcial para a questão 3: 
Corolário 3.40. Seja E um espaço de Banach com FDD incondicional tal que $\mathcal{P}\left({ }^{j} E\right)=$ $\mathcal{P}_{\text {wsc }}\left({ }^{j} E\right), \forall j \in\{1, \ldots, r-1\}$ e $\mathcal{P}\left({ }^{r} E\right) \neq \mathcal{P}_{\text {wsc }}\left({ }^{r} E\right)$. Suponhamos $N$ natural $>r$ e que $P, Q \in \mathcal{P}\left({ }^{N} E\right)$ são tais que $C_{P}=\bigcap_{i=1}^{\infty} P_{i}^{-1}(0)$ e $C_{Q}=\bigcap_{i=1}^{\infty} Q_{i}^{-1}(0)$, onde cada $P_{i}$ é fracamente sequencialmente contínuo de grau $n_{i}$ entre 1 e $N-r$ e cada $Q_{i}$ é fracamente sequencialmente contínuo de grau $m_{i}$ também entre 1 e $N-r$. Então existe $R \in \mathcal{P}\left({ }^{N} E\right)$ $\operatorname{com} C_{R}=C_{P} \cap C_{Q}$.

\section{Demonstração:}

Supondo cada $P_{i}$ e cada $Q_{i} \neq 0$, definimos agora

$$
\begin{aligned}
R(x) & \doteq \sum_{i=1}^{\infty} \frac{P_{i}(x)}{\left\|P_{i}\right\| 2^{i}} \sum_{j \in F_{2 i}} \Phi\left(\sigma_{j}(x)\right) u_{j}^{*}\left(\sigma_{j}(x)\right)^{N-r-n_{i}} \\
& +\sum_{i=1}^{\infty} \frac{Q_{i}(x)}{\left\|Q_{i}\right\| 2^{i}} \sum_{j \in F_{2 i-1}} \Phi\left(\sigma_{j}(x)\right) u_{j}^{*}\left(\sigma_{j}(x)\right)^{N-r-m_{i}}
\end{aligned}
$$

de forma análoga à demonstração da proposição 3.36, e teremos o resultado desejado.

Com raciocínio análogo se prova o corolário 3.41, que responde parcialmente a questão 4:

Corolário 3.41. Seja E um espaço de Banach com FDD incondicional tal que $\mathcal{P}\left({ }^{j} E\right)=$ $\mathcal{P}_{\text {wsc }}\left({ }^{j} E\right), \forall j \in\{1, \ldots, r-1\}$ e $\mathcal{P}\left({ }^{r} E\right) \neq \mathcal{P}_{w s c}\left({ }^{r} E\right)$. Suponhamos $N, M$ naturais maiores que $r$ e que $P, Q \in \mathcal{P}\left({ }^{N} E\right)$ são tais que $C_{P}=\bigcap_{i=1}^{\infty} P_{i}^{-1}(0)$ e $C_{Q}=\bigcap_{i=1}^{\infty} Q_{i}^{-1}(0)$, onde cada $P_{i}$ é fracamente sequencialmente contínuo de grau $n_{i}$ entre 1 e $N-r$ e cada $Q_{i}$ é fracamente sequencialmente contínuo de grau $m_{i}$ entre 1 e $M-r$. Então existe $R \in \mathcal{P}\left({ }^{M+N-r} E\right)$ com $C_{R}=C_{P} \cup C_{Q}$.

\section{Demonstração:}

Supondo novamente cada $P_{i}$ e cada $Q_{i} \neq 0$, definimos

$$
R(x) \doteq \sum_{i, j \in \mathbb{N}} \frac{P_{i}(x) Q_{j}(x)}{\left\|P_{i}\right\|\left\|Q_{j}\right\| 2^{i+j}} \sum_{l \in F_{i j}} \Phi\left(\sigma_{l}(x)\right) u_{l}^{*}\left(\sigma_{l}(x)\right)^{N+M-2 r-n_{i}-m_{j}}
$$

onde $\left\{F_{i j}\right\}_{i, j \in \mathbb{N}}$ é uma partição biindexada dos naturais com cada $F_{i j}$ infinito, e podemos concluir a demonstração, da mesma forma que nos corolários 3.39 e 3.40 . 


\section{Referências Bibliográficas}

[1] Aron, R., On automatic continuity and three problems of "The Scottish Book" concerning the boundedness of polynomial functionals, J. Math. Anal. Appl., 220 (2003), 477-494.

[2] Aron, R. \& Dimant, V., Sets of weak sequential continuity for polynomials, Indag. Mathem., N.S., 13 (3) (2002), 287-299.

[3] Bochnak, J. \& Siciak, J., Polynomials and multilinear mappings in topological vector spaces, Studia Math. 39 (1971), 59-76.

[4] Castillo, J.M.F., Garcia, R. \& Gonzalo, R., Banach spaces in which all multilinear forms are weakly sequentially continuous, Studia Math., 136 (2) (1999), 121-145.

[5] Choi, Y.S. \& Kim, S.G., Polynomial properties of Banach spaces, J. Math. Anal. Appl., 190 (1995), 203-210.

[6] Dimant, V. \& Gonzalo, R., Block diagonal polynomials, Trans. A.M.S. 353 (2000), $733-747$.

[7] Enflo, P., A counterexample to the approximation property in Banach spaces, Acta. Math. 130 (1973), 309-317.

[8] Floret, K., Natural norms on symmetric tensor products of normed spaces, Note di Matematica 17 (1997), 153-188.

[9] Gamelin, T. W., Analytic functions on Banach spaces. Complex potential theory, NATO Adv. Sci. Inst. Ser. C. Math. Phys. Sci., 439, (1994) 187-233.

[10] Gordon, Y. \& Lewis, D. R., Absolutely summing operators and local unconditional structures, Acta. Math. 133 (1974), 27-48. 
[11] Gutiérrez, J.M. Jaramillo, J.A \& Llavona, J.G., Polynomials and geometry of Banach spaces, Extracta Math. 10 (1995) 79-114.

[12] Hissadomi, A. J., Propriedade de Dunford-Pettis polinomial e espaços polinomialmente de Shur, Dissertação de Mestrado, IME-USP, 1998.

[13] Kreyszig, E., Introductory Functional Analysis with Applications, Wiley, New York, 1978.

[14] Maddox, I. J., Elements of Functional Analysis, Cambrige University Press, 1970.

[15] Megginson, R. E., An Introduction to Banach Spaces Theory, Springer, New York, 1998.

[16] Mujica, J., Complex Analysis in Banach Spaces, Math Studies 120, North Holland, Amsterdam (1986)

[17] Phillips, R. S., On linear transformations, Trans. Amer. Math. Soc. 48 (1940) 516541; MR 2, 318.

[18] Pitt, H. R., A note on bilinear forms, J. London Math. Soc. 11 (1936) 174-180.

[19] Rodrigues, L. P., Um teorema de Hahn-Banach para polinômios homogêneos, Dissertação de Mestrado, IME-USP, 2001.

[20] Ryan, R. A., Applications of topological tensor products to infinite dimensional holomorphy, Ph. D. thesis, Trinity College Dublin, 1980.

[21] Schauder, J., Zur Theorie steliger Abbildungen in Funktionalräumen, Math. Z. 26 (1927) 47-65. 